\title{
Dynamische Stabilisierung einer Grenzschichtströmung unter Berücksichtigung nichtlinearer Störausbreitungsprozesse
}

\author{
Dissertation \\ zur Erlangung des Doktorgrades \\ der Mathematisch-Naturwissenschaftlichen Fakultäten \\ der Georg-August-Universität zu Göttingen
}

vorgelegt von

Fabian Evert

aus

Göttingen

Göttingen 2000 
D7

Referent:

Prof. Dr. D. Ronneberger

Korreferent:

Prof. Dr. H. Eckelmann

Tag der mündlichen Prüfung: $\quad$ 2.11.2000 


\section{Kurzfassung}

Es wird die dynamische Stabilisierung einer Grenzschichtströmung am Beispiel einer ebenen Platte untersucht. Hierbei werden mit einem Kompensationsaktuator gezielt künstliche, gegenphasige Störwellen in die Grenzschicht eingebracht, um vorhandene Störungen, deren Anfachung stromab den laminar-turbulenten Umschlag einleitet, zu kompensieren oder zumindest zu dämpfen.

Die zu kompensierenden Primärstörungen werden bei diesen Untersuchungen künstlich, zweidimensional über einen Schlitzaktuator angeregt, wodurch die Abhängigkeit des Kompensationserfolges von der Amplitude der Primärstörung untersucht werden kann.

Die Primärstörung wird von einem Referenzsensor aufgenommen, um daraus im Computer das passende Anregungssignal für den weiter stromab positionierten Kompensationsaktuator zu berechnen. Hierfür muß im wesentlichen das Übertragungsverhalten des physikalischen Ausbreitungsprozesses zwischen dem Referenzsensor und dem Kompensationsaktuator nachgebildet werden. Für diese Nachbildung werden adaptive Digitalfilter eingesetzt, deren Koeffizienten über einen Algorithmus so angepaßt werden, daß die Leistung eines hinter dem Kompensationsaktuator aufgenommenen Fehlersignals minimal wird. Experimente, bei denen diese Übertragungsfunktion mit einem linearen Modell (FIR-Filter) nachgebildet wird, ergeben, daß für höhere Störamplituden zunehmend nichtkompensierbare Reststörungen auftreten. Es wird gezeigt, daß hier insbesondere bei tiefen Frequenzen auch nichtlineare Ausbreitungsprozesse eine Rolle spielen, deren Übertragungsverhalten mit einem FIR-Filter nicht modelliert werden kann.

Ein Teil dieser Übertragungsprozesse kann mit einem Volterra-Filter, das man als Potenzreihenentwichlung mit Gedächtnis betrachten kann, nachgebildet werden. Da der Volterra-Ansatz insbesondere für höhere Potenzen sehr rechenintensiv ist, wird das Vorwissen über den Bandpaßcharakter des Übertragungsprozesses direkt in das Filter integriert, wodurch besonders im nichtlinearen Fall eine deutliche Reduktion der benötigten Koeffizientenanzahl erreicht wird (Kautz-Filter). Im Fall der dynamischen Stabilisierung weisen erste Experimente darauf hin, daß die Integration eines nichtlinearen Modells zu einer verbesserten Kompensation von Störungen großer Amplitude führen kann. 


\section{Inhaltsverzeichnis}

1 Einleitung 1

1.1 Dynamische Stabilisierung . . . . . . . . . . . . . . . 2

2 Experimenteller Aufbau 5

2.1 Windkanal . . . . . . . . . . . . . . . . . 5

2.2 Ebene Platte, Aktuatoren und Sensoren . . . . . . . . . . 6

2.3 Übertragungsverhalten der Aktuatoren . . . . . . . . . . . . . . . 8

2.4 Sensoren . . . . . . . . . . . . . . . . . . . . 9 9

2.5 Hitzdrahtmeßtechnik für Profilmessungen . . . . . . . . . . . 9

2.5.1 Geschwindigkeitsprofile der ungestörten Strömung . . . . . 11

3 Adaptive Signalverarbeitung $\quad 13$

3.1 Adaptive Filter . . . . . . . . . . . . . . . . . . . . . . . . . . 13

3.1 .1 Digitale Filter . . . . . . . . . . . . . . . . . . . . 13

3.1.2 LMS-Algorithmus . . . . . . . . . . . . . . . . . . 15

3.2 Streckenerkennung . . . . . . . . . . . . . . . . . 18

3.3 Dynamische Stabilisierung . . . . . . . . . . . . . . . . . . . 19

3.4 Einfluß unkorrelierter Störkomponenten . . . . . . . . . . . . . . . 22

3.5 Kompensation von elektrischen Einstreuungen . . . . . . . . . . . 24

3.6 Geräte und Programme zur Signalverarbeitung . . . . . . . . . . . 26

4 Stromabausbreitung linear korrelierter Grenzschichtstörungen 29

5 Dynamische Stabilisierung (lineares Modell) 34 
5.1 Versuchsergebnisse bei unterschiedlichen Störamplituden . . . . . 34

5.1.1 Nichtlinearität des Meßverfahrens . . . . . . . . . . . 49

5.2 Einfluß nichtlinearer Störausbreitungsprozesse . . . . . . . . . . . 49

6 Nachbildung nichtlinearer Störausbreitungsprozesse $\quad 56$

6.1 Nichtlineare Modellierung mit einem Volterra-Filter . . . . . . . . 56

6.2 Modellierung mit Vorwissen . . . . . . . . . . . . . . . . 61

6.3 Nichtlineares Kautz-Filter . . . . . . . . . . . . . . . . . 69

7 Dynamische Stabilisierung (nichtlineares Modell) $\quad 73$

8 Zusammenfassung \& Ausblick $\quad 81$

$\begin{array}{lr}\text { A Der filtered-X LMS-Algorithmus } & \mathbf{8 4}\end{array}$

$\begin{array}{lr}\text { Literaturverzeichnis } & \mathbf{8 6}\end{array}$ 


\section{Kapitel 1}

\section{Einleitung}

Die Beeinflussung des laminar-turbulenten Überganges (Transition) bei der Umströmung eines Körpers ist ein Forschungsgegenstand von großer praktischer Bedeutung, da mit der turbulenten Umströmung eine deutliche Erhöhung des Reibungswiderstandes einhergeht. Für Transportflugzeuge im Unterschallbereich wurde gezeigt, daß bis zu $50 \%$ des Gesamtwiderstandes durch Wandreibung induziert sind [17, 4]. Da bei gleicher Reynoldszahl der Reibungswiderstand bei laminarer Umströmung bis zu 90 \% geringer ist als im turbulenten Fall, befassen sich zahlreiche Forschungsprojekte mit der Beeinflussung der laminar-turbulenten Transition.

Insbesondere für Pfeilflügel im transsonischen Bereich sind mehrere Mechanismen für die Turbulenzentstehung verantwortlich. Neben der Staupunktinstabilität ist als erstes die sogenannte Querströmungsinstabilität zu nennen. Sie resultiert aus einem Wendepunkt im spannweitigen Geschwindigkeitsprofil und kann in Abhängigkeit vom Pfeilwinkel schon nach wenigen Zentimetern den laminarturbulenten Umschlag herbeiführen. Verschiedene Untersuchungen haben gezeigt, daß eine Absaugung im Nasenbereich erfolgreich eingesetzt werden kann, um die Querströmungsinstabilität zu unterdrücken und den durch sie induzierten Umschlag zu vermeiden. Bei der Absaugung wird die Stabilisierung durch eine Veränderung des mittleren Geschwindigkeitsprofils erreicht. Ein weiterer Transitionsmechanismus basiert auf Störwellen, die sich stromab in der Grenzschicht ausbreiten, wobei sie in Abhängigkeit von ihrer Frequenz und der Reynoldszahl aufgrund einer viskosen Instabilität gedämpft oder angefacht werden. Ihre Existenz wurde anhand von analytischen Stabilitätsrechnungen zuerst von Tollmien (1929) vorhergesagt, und Schlichting veröffentlichte 1933 Berechnungen zur Größe der Anfachung dieser Intabilitätswellen. Experimentell konnte die Existenz der Tollmien-Schlichting Wellen (TS-Wellen) erst 1947 durch Messungen von Schubauer und Skramstad nachgewiesen werden [33]. Betrachtet man an einer längsangeströmten ebenen Platte die Ausbreitung von zweidimensionalen 
Störwellen, so zeigt sich, daß diese oberhalb einer Indifferenz-Reynoldszahl instabil werden. Oberhalb dieser Reynoldszahl werden die Störungen bei ihrer Ausbreitung stromab in einem gewissen Frequenzband angefacht, wobei sich der Instabilitätsbereich für größere Reynoldszahlen zu tieferen Frequenzen hin verschiebt. Mit zunehmender Störamplitude wird die so gestörte Grundströmung instabil gegenüber sekundären Instabilitäten, die eine dreidimensionale Verformung der Störung mit der Ausbildung $\Lambda$-förmiger Strukturen bewirkt. Die hierauf folgende Entstehung von Turbulenzflecken leitet dann den Übergang zur vollturbulenten Strömung ein. Untersucht man den Einfluß eines Druckgradienten auf den eben beschriebenen Umschlag, so zeigt sich eine stabilisierende Wirkung eines negativen Gradienten, also einer beschleunigten Strömung. Eine Möglichkeit, den durch TS-Wellen induzierten Umschlag zu verzögern, besteht somit in der Konstruktion von sogenannten Laminarprofilen, bei denen die größte Profildicke und damit das Druckminimum weit nach hinten verschoben wird. Die Kombination solcher Laminarprofile mit einer Absaugung im Nasenbereich wird in der Literatur als HLFC (Hybrid Laminar Flow Control) bezeichnet.

\subsection{Dynamische Stabilisierung}

Im Gegensatz zu den bisher erwähnten Techniken, die auf einer Modifizierung des Gleichströmungsprofils beruhen, wird bei dem in dieser Arbeit untersuchten Verfahren versucht, eine Transitionsverzögerung durch die direkte Beeinflussung der instationären Störwellen zu erreichen, deren Anfachung den Umschlag einleitet. Die Grundidee der dynamischen Stabilisierung ist es, in der Grenzschicht künstlich gegenphasige Störungen anzuregen, um so die vorhandenen Instabilitätswellen (z.B. TS-Wellen) zu kompensieren oder zumindest zu dämpfen.

Die ersten Experimente von Milling [27] und Liepmann et al. [24] zeigten, daß eine über einen Störaktuator angeregte, monofrequente TS-Welle stromab durch eine weitere mit einem Kompensationsaktuator eingebrachte Störwelle gedämpft werden kann. Betrag und Phase des Kompensationssignals wurden hierbei über die Minimierung eines weiter stromab aufgenommenen Fehlersignals (Fehlersensor) von Hand optimiert. Beide Untersuchungen wurden an einem Wasserkanal in einer ebenen Plattengrenzschicht durchgeführt, wobei die zweidimensionalen Störwellen über Schwingdrähte bzw. durch periodisches Heizen angeregt wurden. Durch Strömungssichtbarmachung mittels Tinte konnte Milling eine Verschiebung des Ortes abgeschlossener Transition von $R e_{x}=5.2 \cdot 10^{5}$ über die Plattenlänge hinaus $\left(R e_{x}>6.7 \cdot 10^{5}\right)$ als Folge der Störkompensation beobachten. In Hinblick auf die praktische Anwendung der dynamischen Stabilisierung ist es notwendig, den eben beschriebenen Aufbau um einen weiteren Sensor (Referenzsensor) zu erweitern, der stromauf des Kompensationsaktuators in der Grenzschicht vorhandene TS-Wellen aufnehmen kann. Um aus diesem Referenzsignal auch für 
breitbandige Störungen ein geeignetes Kompensationssignal zu berechnen, muß im wesentlichen der physikalische Übertragungsprozeß zwischen der Position des Referenzsensors und der des Kompensationsaktuators nachgebildet werden. In den meisten der im folgenden durchgeführten Untersuchungen jedoch wurde die frequenzabhängige Übertragungsfunktion nur durch ein einfaches Verzögerungsglied und einen frequenzunabhängigen Verstärkungsfaktor nachgebildet. Aus diesem Grund waren die Kompensationserfolge im wesentlichen nur auf einzelne Frequenzen beschränkt [37, 23]. Pupator und Saric erweiterten diese einfache Nachbildung der physikalischen Strecke um eine Filterung des Referenzsignals mit der inversen Übertragungsfunktion des elektromechanischen Signalverarbeitungszweiges. Mit diesem Verfahren war zum ersten Mal auch eine Dämpfung breitbandiger Störungen möglich [30].

Erst Ladd und Hendricks berichten 1988 [22] von einem grundsätzlich neuen Ansatz bei dem der Übertragungsprozeß durch ein lineares, adaptives Digitalfilter nachgebildet wird. Allerdings werden in dieser Arbeit nur Ergebnisse zur Kompensation monofrequenter künstlicher Störungen dargestellt. Bei späteren Experimenten zur Kompensation natürlicher Instabilitätswellen wurde allerdings der Ansatz einer Streckennachbildung durch digitale Filter zugunsten der schon oben beschriebenen Vereinfachung (Verzögerungsglied,Verstärkungsfaktor), kombiniert mit einem einfachen Optimierungsalgorithmus zur Parameteranpassung, wieder fallengelassen, wobei der Grund hierfür aus dem vorliegenden Artikel [21] nicht ersichtlich wird. Einen guten Überblick über die zitierten Arbeiten und weitere Untersuchungen gibt Thomas in [38].

Neuere Experimente zur dynamischen Stabilisierung wurden von Raguse [31] am DLR-Göttingen und Baumann (TU-Berlin) [2] durchgeführt. In beiden Arbeiten wurden digitale Filter als lineares Modell des Störausbreitungsprozesses eingesetzt. Während die Göttinger Untersuchungen an einer ebenen Platte mit künstlichen Breitbandstörungen durchgeführt wurden, konzentrieren sich die Berliner Experimente auf die Kompensation natürlicher TS-Wellen an einem Flügel.

Die in der vorliegenden Arbeit dargestellten Experimente wurden im Anschluß an die Messungen von Raguse am gleichen Modell durchgeführt.

Bei den bisher durchgeführten Untersuchungen zur Kompensation künstlich angeregter Störwellen hat sich gezeigt, daß mit zunehmender Störamplitude keine vollständige Wiederherstellung des ungestörten Strömungszustandes erreicht werden kann. Als eine mögliche Erklärung wird dabei das Auftreten von nichtlinearen Prozessen bei der Störausbreitung angegeben [38, 31].

In dieser Arbeit wird daher untersucht, inwieweit sich diese Prozesse durch Nichtlinearitäten niederer Ordnung beschreiben lassen, und ob durch die Berücksichtigung dieser Nichtlinearitäten eine verbesserte Störkompensation erreicht werden kann. 
Im folgenden Kapitel wird zunächst das bei den Untersuchungen verwendete Plattenmodell zusammen mit den Sensoren und Aktuatoren vorgestellt. Nach einer kleinen Einführung in die Theorie der adaptiven Digitalfilter, wird in Kapitel 3 die Anwendung dieser Filter bei der sogenannten Streckenerkennung und für die dynamische Stabilisierung erläutert. Desweiteren wird der Einfluß von unkorrelierten Störanteilen und sogenannten Nebenwegsstörungen diskutiert.

In Kapitel 4 werden anhand von Streckenerkennungsexperimenten Aussagen zum linearen Übertragungsverhalten der Grenzschicht bzgl. der untersuchten Störungen und der Ausbreitungsgeschwindigkeit dieser Störwellen abgeleitet.

Experimente zur dynamischen Stabilisierung unter Zugrundelegung eines linearen Modells werden in Kapitel 5 diskutiert, wobei insbesondere die Abhängigkeit des Kompensiationserfolges von der Amplitude der zu kompensierenden Störungen untersucht wird.

Mit dem Volterra-Filter wird in Kapitel 6 eine Möglichkeit vorgestellt, daß Übertragungsverhalten der bei den Experimenten zur dynamischen Stabilisierung beobachteten, nichtlinearen Prozesse nachzuzbilden. Da der Volterra-Ansatz sehr rechenintensiv ist, wird mit dem Kautz-Filter eine alternative Filterstruktur untersucht, bei der über die Integration von Vorwissen über die nachzubildene Übertragungsfunktion sowohl im linearen, aber insbesondere auch im nichtlinearen Fall eine deutliche Reduktion der zu adaptierenden Koeffizienten erreicht werden kann.

In Kapitel 8 werden dann erste Ergebnisse zur dynamischen Stabilisierung unter Berücksichtigung der nichtlinearen Ausbreitungsprozesse vorgestellt.

Eine Zusammenfassung findet sich im Abschlußkapitel, wobei auch auf zukünftig zu untersuchende Fragestellungen eingegangen wird. 


\section{Kapitel 2}

\section{Experimenteller Aufbau}

Bei der dynamischen Stabilisierung werden in der Grenzschicht vorhandene instationäre Störungen durch künstlich angeregte, gegenphasige Störwellen kompensiert. Um dieses Verfahren in Abhängigkeit von der Amplitude der zu kompensierenden Störungen untersuchen zu können, werden diese mit einem Störaktuator künstlich zweidimensional angeregt. Hierbei ist darauf zu achten, daß der „natürliche“ Störhintergrund der z.B. durch die Qualität der Anströmung und die Oberflächengüte des umströmten Untersuchungsobjektes bestimmt wird, möglichst gering ist. Zur Detektion der zu kompensierenden Primärstörung wird zwischen dem Störaktuator und dem Kompensationsaktuator ein sogenannter Referenzsensor benötigt, der die Strömung im dahinterliegenden Bereich nicht beeinflussen sollte. Um den Erfolg der dynamischen Stabilisierung beurteilen zu können, wird stromab des Kompensationsaktuators ein weiterer Sensor (Fehlersensor) positioniert, der die nach der Kompensation verbleibenden Reststörungen aufnimmt. Beide Sensoren müssen einen möglichst hohen Signal-Rausch-Abstand aufweisen. Der Störaktuator sollte eine breitbandige Anregung von Störwellen ermöglichen. Auch die Übertragungsfunktion des Kompensationsaktuators darf keine Einbrüche bzw. Nullstellen aufweisen, da bei den jeweiligen Frequenzen sonst keine Kompensation möglich ist.

\subsection{Windkanal}

Die Experimente der vorliegenden Arbeit wurden im kleinen Laminarkanal des DLR Göttingen durchgeführt. Es handelt sich hierbei um einen kontinuierlich betriebenen Niedergeschwindigkeitswindkanal Eiffelscher Bauweise mit einer offenen Meßstrecke und Luftrückführung durch die Halle. Die Freistrahldüse hat einen Querschnitt von 0, 4·0, $4 \mathrm{~m}^{2}$ bei einem Potentialkerndurchmesser von 0,3 m. Die Geschwindigkeit konnte bis zu einem Maximalwert von etwa $21 \frac{\mathrm{m}}{\mathrm{s}}$ stufenlos 


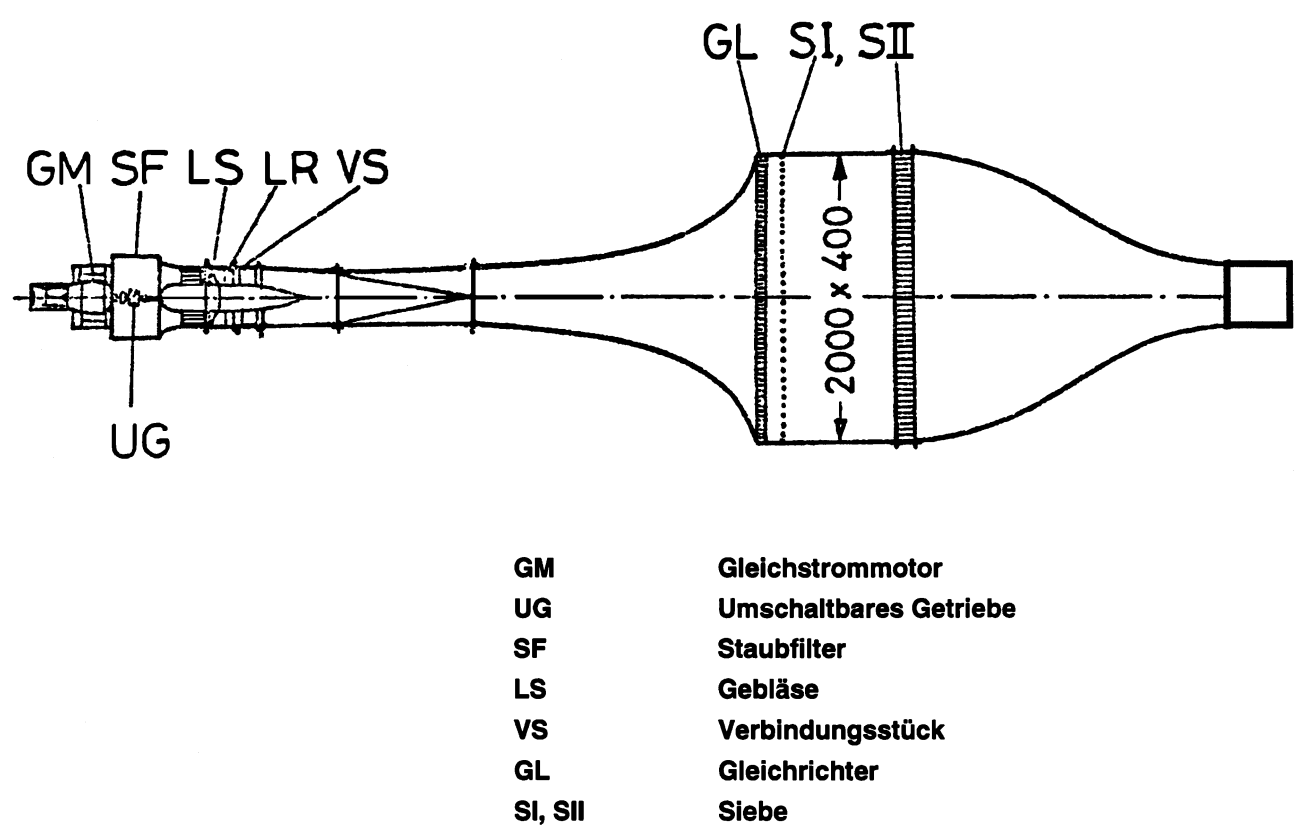

Abbildung 2.1: Grundriß des kleinen Laminarkanals

geregelt werden. In Abbildung 2.1 ist der Grundriß des Kanals dargestellt.

\subsection{Ebene Platte, Aktuatoren und Sensoren}

Als Untersuchungsobjekt wurde eine ebene Platte mit einem Aktuatorensystem gewählt, das seine Eignung zur Störungsanregung und Kompensation monofrequenter Störungen bereits in Prinzipuntersuchungen gezeigt hat [41, 13]. Der Aufbau ist schematisch in den Abbildungen 2.2 und 2.3 dargestellt.

Das Modell besteht aus einer ebenen Holzplatte, sowie aus zwei Seitenwänden und einem justierbaren Deckel aus Plexiglas. Die Vorderkante der Platte und der Plexiglasplatten sind halbelliptisch geformt, um vorzeitigen Strömungsablösungen entgegenzuwirken. Für die Holzplatte beträgt das Achsenverhältnis 6:1.

Die Anregung der Störwellen erfolgt durch alternierendes Einsaugen und Ausblasen von Grenzschichtfluid. Zu diesem Zweck sind in die Platte quaderförmige Kammern mit scharfkantigen Schlitzöffnungen eingebaut. Diese Kammern sind auf der Plattenunterseite über Plastikschläuche mit Druckkammerlautsprechern verbunden. Die Kammer für die Störanregung (Nr.1 in Abb. 2.2) mit fünf Zuleitungen hat ein Volumen von 16,5 $29 \cdot 200 \mathrm{~mm}^{3}$, während die zwanzig Kammern des Kompensationsaktuatorarrays (Nr.3 in Abb. 2.2) mit jeweils zwei Zuführungen ein Volumen von $5 \cdot 12 \cdot 19 \mathrm{~mm}^{3}$ aufweisen. 
Alle Maße in mm, nicht maßstabsgetreu

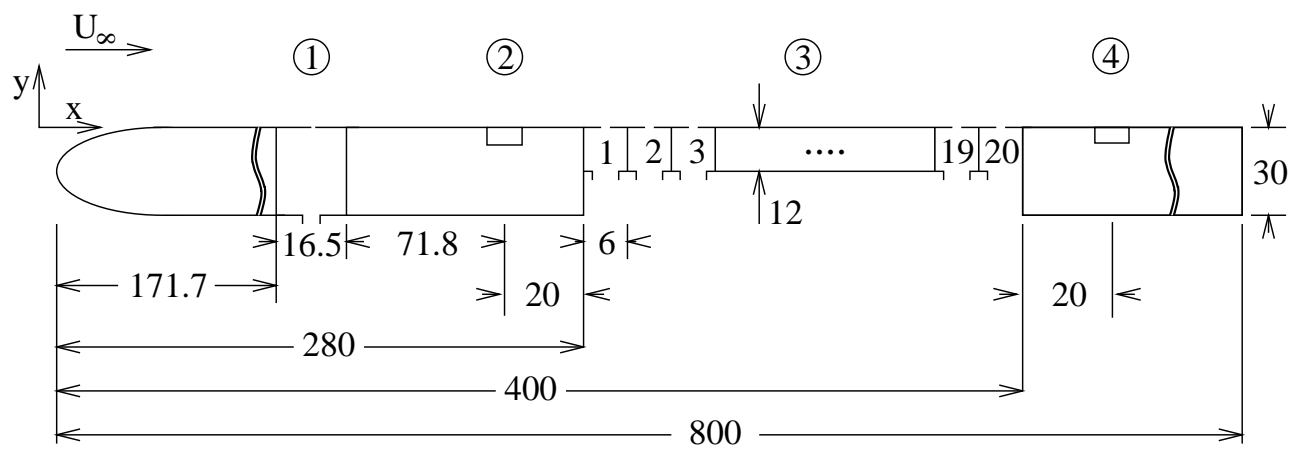

1: Störaktuator 2: Referenzsensoren 3: Aktuatorenarray 4: Fehlersensor

Abbildung 2.2: Aufbau der Platte (Schnitt)

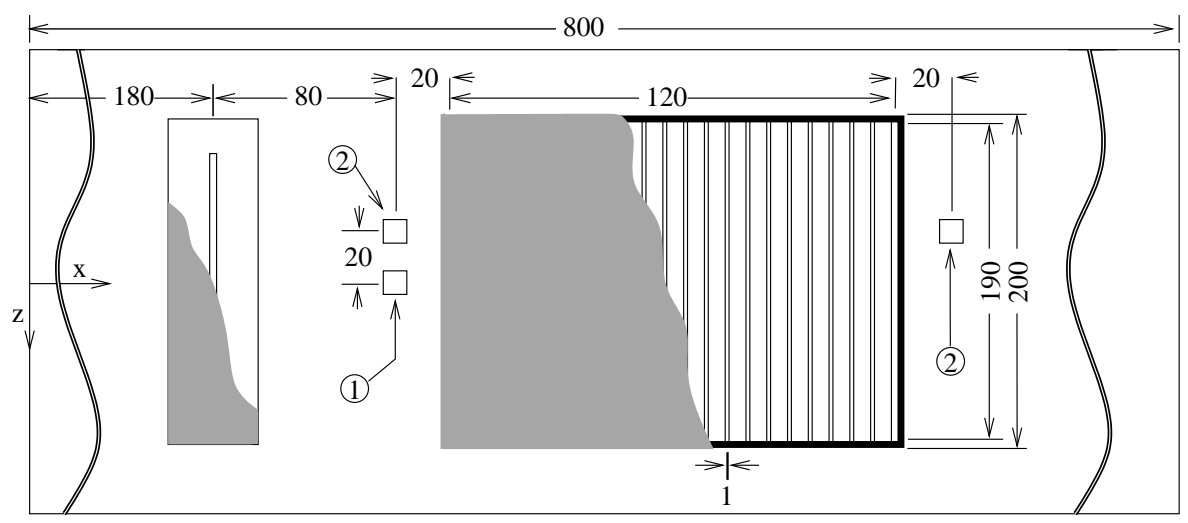

Alle Maße in mm, nicht maßstabsgetreu

1: Heißfilmsensor 2:Oberflächenhitzdrähte

Abbildung 2.3: Aufbau der Platte (Draufsicht) 


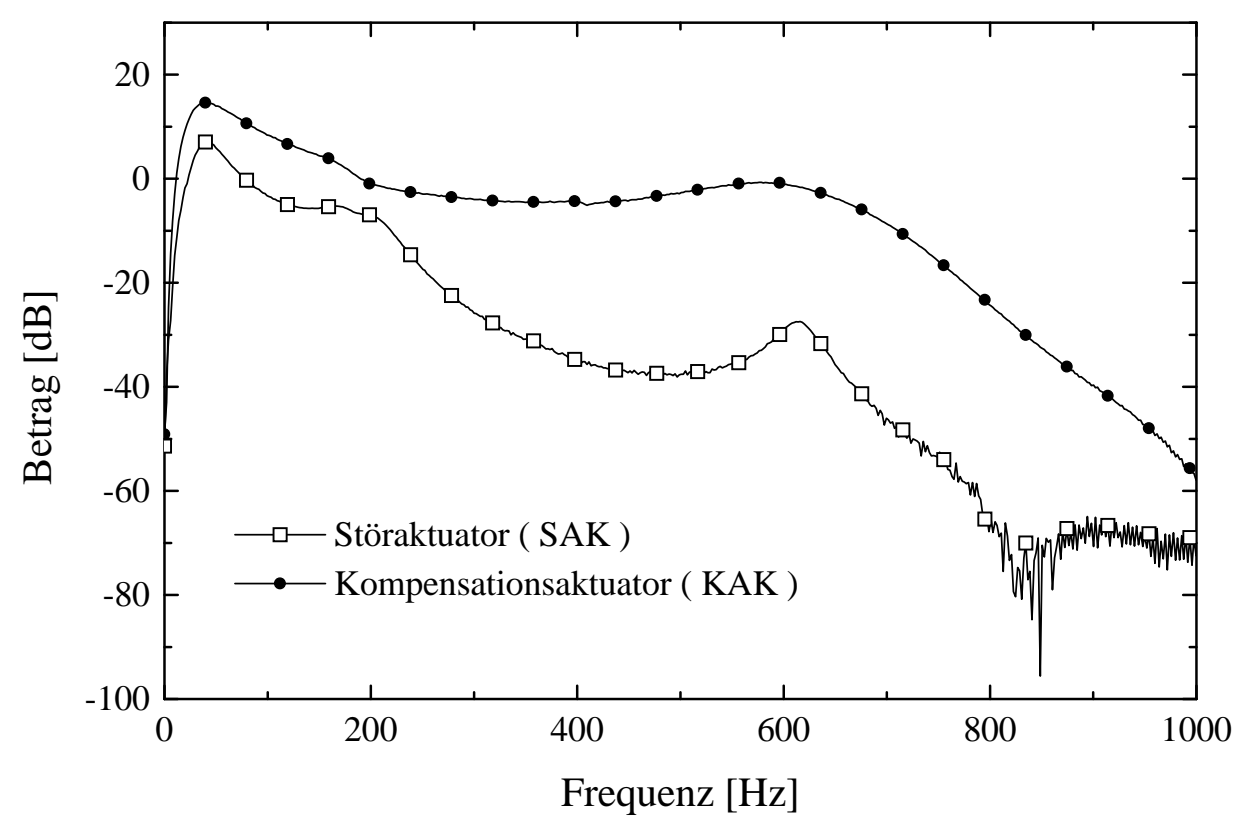

Abbildung 2.4: Betrag der Übertragungsfunktion zwischen der Rauschanregung und dem Störsignal im Nahfeld des Aktuators

Um störende Einflüsse der Schlitze (ohne Anregung) auf die Strömung zu verhindern, sind sie mit einem Metalldrahtgewebe (Porosität: 25\%) überzogen. Das Gewebe schließt bündig mit der Plattenoberfläche ab.

\section{3 Übertragungsverhalten der Aktuatoren}

Zur Erzeugung breitbandiger Störwellen wurde als Anregungssignal für den Störaktuator eine Folge von statistisch unabhängigen Zahlen generiert, die zwischen $\pm A$ gleichverteilt sind, wobei $A$ die Störamplitude bezeichnet. Im folgenden wird diese Signalform als weißes Rauschen bezeichnet. Das Aktuatorsystem stellt mit der Aktuatorkammer, den Zuführungsschläuchen und Verteilerelementen jedoch ein Resonanzsystem dar, das zu einer spektralen Einfärbung des Störsignals am Schlitz führt. In Abbildung 2.4 ist der Betrag der Übertragungsfunktion zwischen der Rauschanregung und dem resultierenden Störsignal im Nahfeld des Aktuatorschlitzes aufgetragen. Das Störsignal wurde mit einem Hitzdraht etwa $1 \mathrm{~mm}$ über dem Schlitz aufgenommen und die Übertragungsfunktion mittels einer Streckenerkennung bestimmt (siehe Abschnitt 3.2). Insbesondere für den Störaktuator zeigt sich eine deutliche spektrale Färbung mit einem starken Abfall der übertragenen Leistung für Frequenzen über $200 \mathrm{~Hz}$. Bei den in dieser Arbeit betrachteten Reynoldszahlen liegt der Instabilitätsfrequenzbereich jedoch zwischen 
etwa $50 \mathrm{~Hz}$ und $220 \mathrm{~Hz}$. Hier kann sicher noch von einer breitbandigen Anregung gesprochen werden. Wichtiger ist jedoch, daß der Kompensationsaktuator in diesem Frequenzbereich ein relativ glattes Übertragungsverhalten ohne Nullstellen aufweist, da anderenfalls bei den entsprechenden Frequenzen die Steuerbarkeit des Systems nicht gewährleistet wäre.

\subsection{Sensoren}

In den ersten Experimenten wurde ein auf der Mittelachse des Modells eingebauter Heißfilmwandsensor zur Aufnahme des Referenzsignales verwendet und der Restfehler mit einem frei positionierbaren Hitzdrahtsensor bestimmt. Insbesondere für die Untersuchung nichtlinear generierter Störanteile erwies sich jedoch der Signal-Rausch-Abstand des Heißfilmsensors als zu gering. An der TU-Berlin wurden sogenannte Oberflächenhitzdrähte entwickelt und gefertigt, die sich durch einen deutlich höheren Signal-Rausch-Abstand auszeichnen, als der anfänglich verwendete Heißfilmsensor. Diese Verbesserung ist auf eine verringerte Wärmeleitung in die Wand zurückzuführen, die durch einen kleinen Schlitz erreicht wird, in dem ein Hitzdraht oberflächenbündig gespannt ist. Außerdem können diese Sensoren mit einem höheren Überhitzungsverhältnis betrieben werden als Heißfilmelemente. In Abbildung 2.5 ist der prinzipielle Aufbau eines solchen Sensors dargestellt. Für die in dieser Arbeit beschriebenen Experimente wurden zwei dieser Sensoren als Referenz- bzw. Fehlersensor in die Platte eingebaut (siehe Abbildung 2.3). Die Sensoren wurden mit einem Überhitzungsverhältnis von ca. $\frac{R}{R_{0}}=1,7 \mathrm{im}$ Konstant-Temperatur-Verfahren betrieben (Dantek 56C17 CTA-Brücke), wobei $R$ den elektrischen Sensorwiderstand bei der Überhitzungstemperatur und $R_{0}$ den Widerstand bei der Fluidtemperatur bezeichnet. Die Ausgangsspannung des Anemometers ist hierbei eine Funktion der Wandschubspannung $\tau_{\mathrm{W}}[2,7]$.

\subsection{Hitzdrahtmeßtechnik für Profilmessungen}

Zur Messung der mittleren Geschwindigkeit $\bar{U}$ und der Schwankungskomponente $u$ in Richtung der Anströmung wurde eine Hitzdrahtsonde verwendet.

Diese konnte tangential zur Plattenoberfläche $(x-z$-Ebene) von Hand, mittels eines Zwei-Komponenten-Verschiebegerätes positioniert und in wandnormaler Richtung ( $y$-Koordinate) über eine Schrittmotorsteuerung $(\Delta y=1 \mu \mathrm{m})$ automatisch verfahren werden.

Bei der Hitzdrahtmessung wird aus der pro Zeiteinheit von einem dünnen, elektrisch geheizten Draht an das Medium abgegebenen Wärmemenge $\dot{Q}$ auf den Massenfluß des umgebenden Fluids geschlossen. 


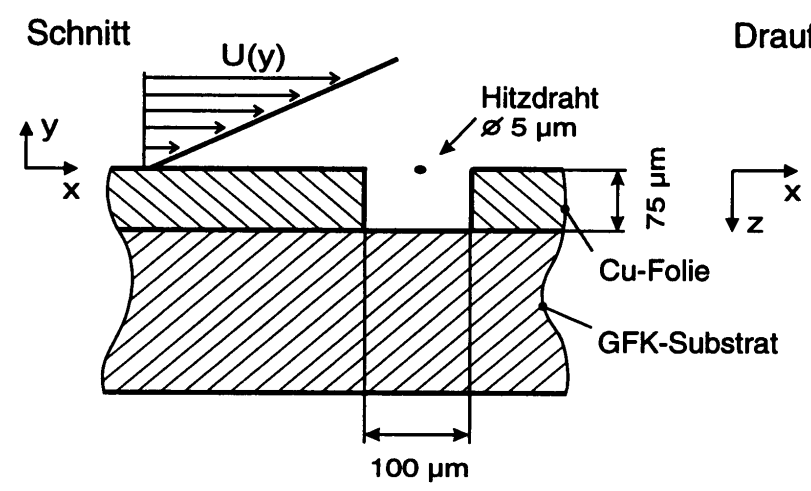

\section{Draufsicht}

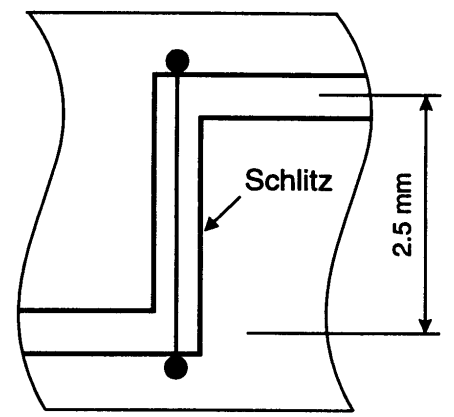

Abbildung 2.5: Prinzipieller Aufbau eines Oberflächenhitzdrahtsensors (aus Baumann [2])

Bei dem hier verwendeten Konstant-Temperatur-Verfahren wird der Sensorwiderstand und damit auch dessen Temperatur mit einer schnellen Widerstandsmeßbrücke unabhängig von der Strömungsgeschwindigkeit konstant gehalten.

Anhand des Kingschen Gesetzes kann der nichtlineare Zusammenhang zwischen der Strömungsgeschwindigkeit $U_{\perp}$ normal zur Hitzdrahtachse und der Anemometerausgangsspannung E durch

$$
\mathrm{E}^{2}=\mathrm{A}^{*}+\mathrm{B}^{*} \mathrm{U}_{\perp}^{1 / \mathrm{n}}
$$

beschrieben werden [7]. Um die Konstanten $\mathrm{A}^{*}, \mathrm{~B}^{*}$ und $\mathrm{n}$ zu bestimmen, wurde vor den eigentlichen Messungen jeweils eine Eichung durchgeführt. Hierbei wird der Hitzdraht in der Meßstrecke außerhalb der Grenzschicht in der Nähe des zur Bestimmung der Anströmungsgeschwindigkeit $U_{\infty}$ verwendeten PrandtlRohres positioniert und dann die Anemometerausgangsspannung für unterschiedliche Strömungsgeschwindigkeiten bestimmt. Aus den so erhaltenen Wertepaaren $\left(\bar{U}_{\perp} ; E^{2}\right)$ wurden die Konstanten aus Gl. 2.1 über die Minimierung des quadratischen Fehlers ermittelt.

Der Nullpunkt der Ausgangsspannung des Anemometers wurde verschoben und die Differenz so verstärkt, daß der Eingangsspannungsbereich $( \pm 5 \mathrm{~V})$ des bei der Signalaufnahme verwendeten 12 bit Analog-Digital-Wandlers bestmöglich genutzt wurde. Für Profilmessungen konnte die Positionierung der Hitzdrahtsonde (y-Koordinate) und die Aufnahme der Anemometerausgangsspannung für einen angegebenen Koordinatensatz mit einem Computerprogramm vollautomatisch gesteuert werden. An jedem Meßpunkt wurde das Spannungssignal mit der Abtastrate $f_{s}=1 / \Delta t=2 \mathrm{KHz}$ für eine Meßzeit von $\mathrm{T} \approx 10 \mathrm{~s}$ aufgenommen. Aus diesen Signalen konnte dann über Gl. 2.1 die Strömungsgeschwindigkeit $U$ berechnet werden. Die Messungen der Schwankungsgeschwindigkeit haben gezeigt, daß es im tieffrequenten Bereich für Frequenzen kleiner $50 \mathrm{~Hz}$ neben einer Son- 
denschwingung weitere starke Störkomponenten gab, die weitgehend unabhängig vom Wandabstand und der künstlichen Störanregung waren. Da diese Störungen den Effektivwert (RMS) der Geschwindigkeitsschwankungen stark verfälschen, wurden bei dessen Berechnung die Frequenzkomponenten unterhalb von $50 \mathrm{~Hz}$ unterdrückt.

\subsubsection{Geschwindigkeitsprofile der ungestörten Strömung}

Das Stabilitätsverhalten der TS- Wellen ist in hohem Maße von der Form des Geschwindigkeitsprofils in der Grenzschicht bzw. vom Druckverlauf in x-Richtung abhängig. Während ein negativer Druckgradient eine stabilisierende Wirkung hat, beschleunigt ein positiver Druckgradient den laminar-turbulenten Umschlag. Desweiteren hat das Geschwindigkeitsprofil im Fall eines positiven Druckgradienten einen Wendepunkt und ist daher, nach dem Wendepunktkriterium, auch bei Vernachlässigung des Reibungseinflusses instabil (reibungslose Instabilität, [33]). Für die Untersuchung der viskosen TS-Instabilität wurde daher eine zweidimensionale Blasius-Strömung (ohne Druckgradient) vorausgesetzt.

Anstelle des Druckgradienten wurde der axiale Geschwindigkeitsgradient mittels Hitzdrahtmessungen bestimmt und die obere Plexiglasabdeckung so justiert, daß dieser zwischen dem Störaktuator und dem Fehlersensor minimal wurde. Da die Grenzschichtdicke und damit auch ihre Verdrängungswirkung nicht linear mit der Lauflänge zunimmt, sondern proportional zur Wurzel aus der Lauflänge ist, kann eine Strömung ohne Druckgradienten jedoch nicht für die gesamte Meßstrecke erreicht werden.

Es wurden nun an 6 Positionen stromab $(\Delta x=50 \mathrm{~mm})$, beginnend beim Störaktuator (Nr. 1 in Abb. 2.2), Gleichströmungsprofile bestimmt. Die letzte Meßposition befand sich $1 \mathrm{~cm}$ hinter dem Fehlersensor (Nr. 4 in Abb. 2.2). Die Außenströmungsgeschwindigkeit betrug hier, wie bei allen weiteren Messungen $U_{\infty}=11 \frac{\mathrm{m}}{\mathrm{s}}$. Für einen Vergleich der Ergebnisse mit der Blasius-Lösung ist zu beachten, daß die Beschleunigung der Strömung an der Plattenvorderkante eine im Vergleich mit der Blasius-Lösung verzögerte Grenzschichtentwicklung bewirkt. Daher wurden die erhaltenen Geschwindigkeitsprofile über die Minimierung des mittleren quadratischen Fehlers an die Ähnlichkeitslösung von Blasius angepaßt, wobei als freier Parameter eine Verschiebung $(\Delta x)$ der Meßposition verwendet wurde. Aus diesen Rechnungen ergibt sich die virtuelle Lauflänge mit einer Unsicherheit von $\pm 4 \mathrm{~mm}$ zu $x_{V}=x-27 \mathrm{~mm}$. Für die Berechnung der im folgenden angegebenen Reynoldszahlen wurde die virtuelle Laufänge zugrundegelegt.

Die sich aus diesen Messungen ergebenen Gleichströmungsprofile sind in Abbildung 2.6 bezogen auf die mittlere Strömungsgeschwindigkeit am Grenzschichtrand $U_{e}$ dargestellt. Die $y$-Koordinate wurde mit der Blasius-Länge $\delta_{r}=$ $\sqrt{\nu x_{V} / U_{e}}$ entdimensionalisiert $\left(\eta=y / \delta_{r}\right)$. Die gemessenen Profile weisen keinen 


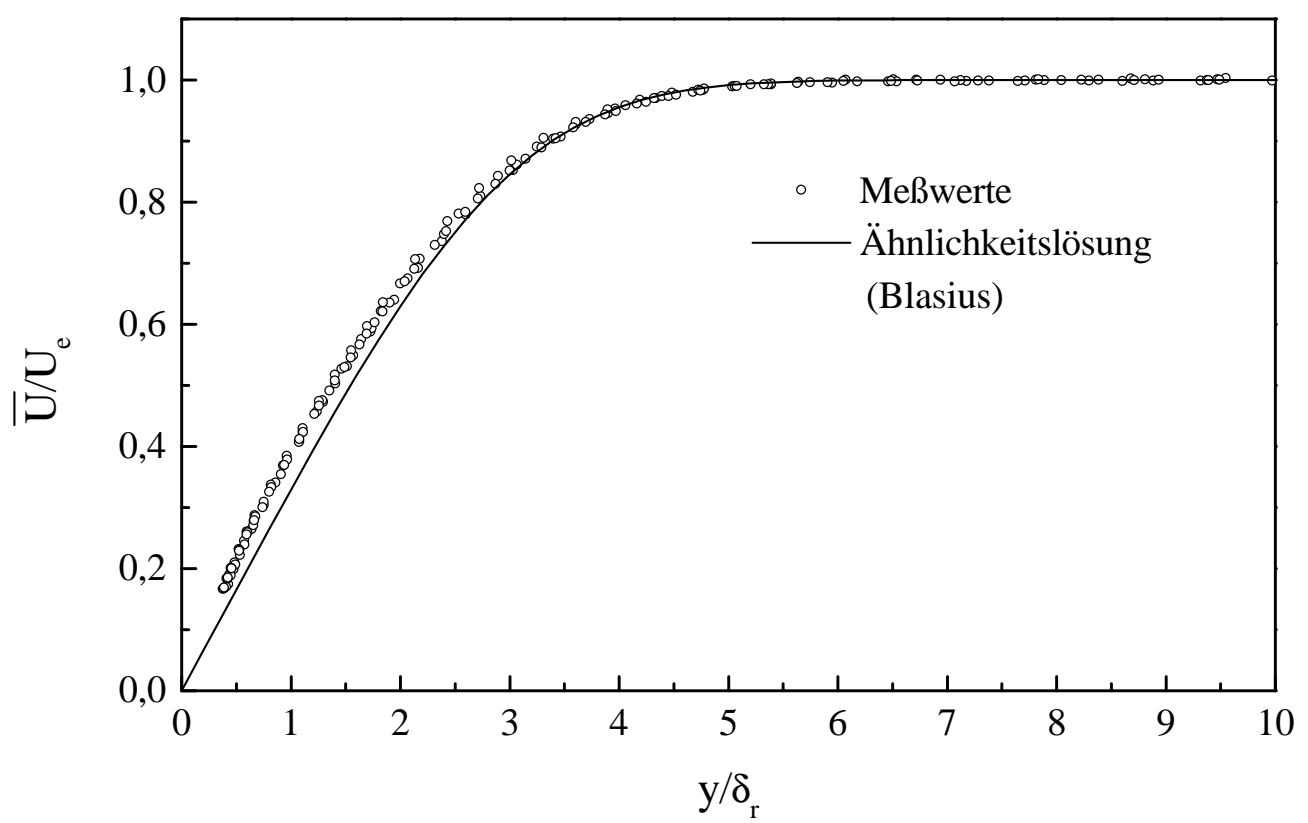

Abbildung 2.6: Geschwindigkeitsprofile für 6 Messungen, $x_{V}=$ $153 \mathrm{~mm} \cdots 403 \mathrm{~mm}(\Delta x=50 \mathrm{~mm})$

Wendepunkt auf, wobei die Ursache für die Abweichung von der Blasius-Lösung nicht geklärt werden konnte. Da jedoch die Frequenzbereiche, für die eine Störanfachung beobachtet wurde, relativ gut mit der theoretischen Erwartung für eine Blasius-Grenzschicht übereinstimmen, ist zu vermuten, daß diese Abweichung zumindest teilweise auf ein Meßproblem zurückzuführen ist. 


\section{Kapitel 3}

\section{Adaptive Signalverarbeitung}

Bei der dynamischen Stabilisierung werden die in der Grenzschicht vorhandenen Störungen mit dem Referenzsensor (RS) aufgenommen, um hiermit ein geeignetes Eingangssignal für den Kompensationsaktuator (KAK) zu berechnen. Hierfür werden adaptive digitale Filter eingesetzt. Nach einer Einführung in die Theorie linearer, adaptiver Filter werden in diesem Kapitel die Prinzipien der Systemerkennung und der dynamischen Stabilisierung dargestellt. Danach wird der in der Praxis nicht zu vernachlässigende Einfluß unkorrelierter Rauschkomponenten und sogenannter korrelierter Nebenwegsstörungen auf die dynamische Stabilisierung erläutert. Abschließend werden die zur Signalverarbeitung verwendeten Geräte und Programme beschrieben.

\subsection{Adaptive Filter}

Zur Berechnung des Kompensationssignals wird durch ein adaptives Digitalfilter im wesentlichen die physikalische Übertragungsfunktion zwischen dem Ort des Referenzsensors und der Position des Kompensationsaktuators nachgebildet. Im folgenden wird die in den Experimenten verwendete lineare Filterstruktur und der Algorithmus zur Adaption der Filterkoeffizienten vorgestellt.

\subsubsection{Digitale Filter}

Ein digitales Filter erzeugt zu jedem diskreten Zeitpunkt $n$ aus dem Eingangssignalvektor

$$
\mathbf{x}[n]=(x[n], x[n-1], \ldots, x[n-N+1])^{T}
$$

ein Ausgangssignal $y[n]$. Hier beschreibt ein solcher Signalvektor ein an äquidistanten Zeitpunkten $n \cdot \Delta t$ abgetastetes, digitalisiertes Signal, wobei $x[n]$ der 
aktuelle Abtastwert ist und $x[n-i]$ dem Wert entspricht, der $i$ Zeitschritte zuvor abgetastet wurde. Eine wichtige Klasse von linearen, kausalen Filtern wird durch die Differenzengleichung

$$
y[n]=\sum_{i=0}^{N-1} a_{i} x[n-i]+\sum_{j=1}^{M} b_{j} y[n-j]
$$

beschrieben, wobei die Koeffizienten $a_{i}$ den transversalen und die Koeffizienten $b_{j}$ den rekursiven Anteil parametrisieren. Die Übertragungsfunktion ergibt sich mittels der z-Transformation als

$$
H(z)=\frac{Y(z)}{X(z)}=\frac{\sum_{i=0}^{N-1} a_{i} z^{-i}}{1-\sum_{j=1}^{M} b_{j} z^{-j}}
$$

mit den $z$-Transformierten von $x[n]$ bzw. $y[n]$

$$
X(z)=\sum_{n=0}^{\infty} x[n] z^{-n} \text { und } Y(z)=\sum_{n=0}^{\infty} y[n] z^{-n}, z \in \mathbb{C} .
$$

Durch die Faktorisierung der Übertragungsfunktion ergibt sich die bis auf den Faktor $A$ eindeutige Darstellung des Filters durch seine Pole $d_{j}$ und Nullstellen $c_{i}$ in der komplexen Ebene.

$$
H(z)=A \frac{\prod_{i=0}^{N-1}\left(1-c_{i} z^{-1}\right)}{\prod_{j=0}^{M}\left(1-d_{j} z^{-1}\right)}
$$

Damit $H(z)$ kausal und stabil in dem Sinne ist, daß das Ausgangssignal bei begrenzten Eingangssignalen endlich bleibt (Bounded Input-Bounded OutputStabilität) müssen alle Pole $d_{j}$ innerhalb des Einheitskreises liegen. Eine weitere Beschreibungsmöglichkeit ist die sogenannte Impulsantwort des Filters, also das Ausgangssignal bei Anregung mit dem Eingangssignal [1, 0, 0, ․ ] . Für Filter, die durch Gleichung 3.2 beschrieben werden, ist diese durch den rekursiven Anteil unendlich lang, weshalb diese Filter IIR-Filter (Infinite Impulse Response) genannt werden. Sind alle rekursiven Koeffizienten gleich Null, so entspricht die Impulsantwort gerade den transversalen Koeffizienten. Solche Filter werden als Transversalfilter oder FIR-Filter (Finite Impulse Respone) bezeichnet. Die Fouriertransformierte der Impulsantwort liefert die Übertragungsfunktion im Frequenzbereich (Frequenzgang). Dies entspricht einer Auswertung der z-Transformierten auf dem Einheitskreis. Prinzipiell kann jede Übertragungsfunktion, die durch ein IIR-Filter gegeben ist, auch durch ein FIR-Filter approximiert werden, jedoch kann die Anzahl der benötigten Koeffizienten sehr viel größer sein. Dem Vorteil einer möglichen Koeffizientenersparnis bei der Verwendung von IIR-Filtern stehen jedoch einige Nachteile, wie z.B. die nicht gesicherte Stabilität, gegenüber, weshalb in dieser Arbeit nur adaptive FIR-Filter verwendet wurden.

Weiterführende Darstellungen zur Theorie der digitalen Filter finden sich z.B. in [29] und [20]. 


\subsubsection{LMS-Algorithmus}

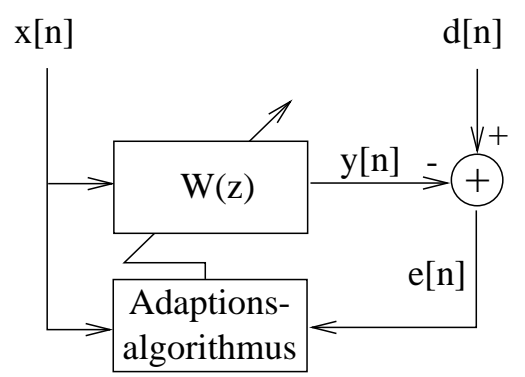

Abbildung 3.1: Adaptives Filter

In Abbildung 3.1 ist ein adaptives Filter, bestehend aus einem FIR-Filter und einem Algorithmus zur Anpassung der Koeffizienten dargestellt. Die Aufgabe des Adaptionsalgorithmus ist es, die Filterkoeffizienten über die Minimierung einer vorgegebenen Kostenfunktion optimal einzustellen.

Als Ausgangssignal des FIR-Filters mit den Koeffizienten

$$
\mathbf{w}=\left(w_{0}, w_{1}, \ldots, w_{N-1}\right)^{T}
$$

ergibt sich

$$
\begin{aligned}
y[n] & =\sum_{i=0}^{N-1} w_{i} x[n-i] \\
& =\mathbf{w}^{T} \mathbf{x}[n]=\mathbf{x}^{T}[n] \mathbf{w} .
\end{aligned}
$$

Für den Fehler $e[n]$, der sich als Differenz zwischen dem sogenannten desiredSignal $d[n]$ und dem Ausgangssignal des Filters ergibt, erhält man somit

$$
\begin{aligned}
e[n] & =d[n]-y[n] \\
& =d[n]-\mathbf{x}^{T}[n] \mathbf{w} .
\end{aligned}
$$

Kostenfunktion $J[n]$ ist in der Regel der Erwartungswert des quadrierten Fehlers, dessen Minimierung zum optimalen Koeffizientenvektor $\mathbf{w}^{o}$ führt.

$$
\begin{aligned}
J[n] & =\left\langle e^{2}[n]\right\rangle \\
& =\left\langle d[n]^{2}\right\rangle-2 \mathbf{w}^{T}\langle d[n] \mathbf{x}[n]\rangle+\mathbf{w}^{T}\left\langle\mathbf{x}[n] \mathbf{x}^{T}[n]\right\rangle \mathbf{w}
\end{aligned}
$$

Mit der Autokorrelationsmatrix

$$
\mathbf{R}=\left\langle\mathbf{x}[n] \mathbf{x}^{T}[n]\right\rangle
$$


und dem Kreuzkorrelationsvektor

$$
\mathbf{p}=\langle d[n] \mathbf{x}[\mathbf{n}]\rangle
$$

lautet die Kostenfunktion

$$
J[n]=\left\langle d[n]^{2}\right\rangle-2 \mathbf{p}^{T} \mathbf{w}+\mathbf{w}^{T} \mathbf{R} \mathbf{w} .
$$

Man sieht, daß die Kostenfunktion eine quadratische Form in den Filterkoeffizienten ist, wodurch das Minimum bezüglich der Koeffizienten eindeutig bestimmt ist. Der optimale Koeffizientenvektor $\mathbf{w}^{o}$ ergibt sich durch das Verschwinden des Gradienten bzgl. w im Minimum mit

$$
\nabla J[n]=2 \mathbf{R w}-2 \mathbf{p}
$$

und

$$
\nabla J[n]=2 \mathbf{R w}^{o}-2 \mathbf{p} \stackrel{!}{=} 0
$$

als Lösung der Gleichung

$$
\mathbf{R w}^{o}=\mathbf{p}
$$

Dies ist die diskrete Form der Wiener-Hopf-Gleichung. Die Autokorrelationsmatrix $\mathbf{R}$ ist eine positiv-semidefinite Toeplitz-Matrix. Für ein spektral hinreichend reichhaltiges Eingangssignal $\mathbf{x}[n]$ ist sie positiv definit und damit invertierbar, wodurch sich das optimale Filter (Wienersches Optimalfilter) über

$$
\mathbf{w}^{o}=\mathbf{R}^{-1} \mathbf{p}
$$

ergibt. Mit der Fouriertransformation erhält man als Übertragungsfunktion im Frequenzbereich

$$
W^{o}(f)=\frac{S_{d, x}(f)}{S_{x, x}(f)}
$$

Hier wird die Forderung nach einem Eingangssignalspektrum ohne Nullstellen sofort verständlich. Obwohl für die Invertierung von Toeplitz-Matrizen effiziente Algorithmen zur Verfügung stehen, ist die Berechnung für viele Echtzeitanwendungen zu komplex. Dies gilt insbesondere unter instationären Bedingungen, da hier eine kontinuierliche Schätzung der Parameter erforderlich ist.

Aufgrund der quadratischen Form der Kostenfunktion kann der optimale Koeffizientenvektor jedoch auch durch eine schrittweise Annäherung in Richtung des negativen Gradienten der Kostenfunktion (Gl. 3.16) gefunden werden. 
Dies führt zu folgendem Algorithmus:

$$
\begin{aligned}
\mathbf{w}[n+1] & =\mathbf{w}[n]-\frac{1}{2} \mu \nabla J[n] \\
& =\mathbf{w}[n]-\mu \mathbf{R} \mathbf{w}[n]+\mu \mathbf{p}
\end{aligned}
$$

Bei der Methode des steilsten Abstieges ist zwar keine Matrixinvertierung mehr notwendig, jedoch müssen $\mathbf{R}$ und $\mathbf{p}$ immer noch geschätzt werden. Eine weitere Vereinfachung ergibt sich, wenn man als Kostenfunktion nicht den Erwartungswert, sondern den Momentanwert des mittleren quadratischen Fehlers $e^{2}[n]$ verwendet. Für den Gradientenschätzer ergibt sich somit

$$
\nabla J[n]=-2 \mathbf{x}[n] e[n]
$$

und die Iterationsvorschrift (Gl. 3.22) wird zu

$$
\mathbf{w}[n+1]=\mathbf{w}[n]+\mu \mathbf{x}[n] e[n]
$$

Dies ist der von Widrow [39] eingeführte LMS-Algorithmus (Least Mean Squares). Betrachtet man diese Vorschrift, so wird deutlich, daß in den Filterkoeffizienten eigentlich die Korrelation zwischen dem Fehlersignal $e[n]$ und dem Eingangssignal $x[n-i]$ für die Zeitverschiebungen $i=0,1, \cdots N-1$ gebildet wird. Im Ausgangssignal des FIR-Filters, das mit diesen Koeffizienten berechnet wird, finden sich somit die Anteile des Fehlersignals, die mit dem Eingangssignal korreliert sind. Diese werden bei der Fehlerbildung vom desired-Signal abgezogen, was dazu führt, daß Fehlersignal und Eingangssignal durch den Algorithmus dekorreliert werden. Wichtig ist eine geeignete Wahl des Schrittweitenparameters $\mu$, da dieser neben der Konvergenzgeschwindigkeit und dem Restfehler auch die Stabilität des iterativen Verfahrens bestimmt. Um die Konvergenz zu gewährleisten, muß gelten

$$
0<\mu<\frac{2}{N P_{x}}
$$

wobei $P_{x}=\left\langle x^{2}[n]\right\rangle$ die Leistung des Eingangssignals ist. Da der LMS-Algorithmus eine numerische Komplexität von $O(N)$ besitzt und sich numerisch sehr gutmütig verhält, hat er eine große Verbreitung erlangt. Ein Nachteil ist jedoch die Abhängigkeit des Konvergenzverhaltens von der Eingangssignalstatistik. Es läßt sich zeigen, daß der LMS um so langsamer konvergiert, je größer die Eigenwertspreizung, also das Verhältnis vom größten Eigenwert zum kleinsten Eigenwert, der Autokorrelationsmatrix ist. Für die praktische Anwendung ist diese Aussage wenig hilfreich, jedoch haben Narayan et al. [35] gezeigt, daß die Eigenwertspreizung durch

$$
1 \leq \frac{\lambda_{\max }}{\lambda_{\min }} \leq \frac{\max |\mathrm{X}(\mathrm{f})|^{2}}{\min |\mathrm{X}(\mathrm{f})|^{2}}
$$


anhand der spektralen Dynamik abgeschätzt werden kann. $X(f)$ bezeichnet hierbei die Fouriertransformierte des Eingangssignals. Obwohl die Signale bei den hier durchgeführten Untersuchungen eine starke spektrale Färbung aufweisen und der LMS somit eine verminderte Konvergenz aufweist, wurde er bei der dynamischen Stabilisierung und zur Adaption der untersuchten nichtlinearen Modelle aufgrund seiner geringen numerischen Komplexität und großen Robustheit verwendet.

Für Reihenmessungen in Streckenerkennungskonfiguration wurde aus Gründen der Zeitersparnis der SFTF (Stabilized Fast Transversal Filter) [34] eingesetzt. Dieser Algorithmus zeichnet sich durch eine schnelle, von der Eingangssignalstatistik weitgehend unabhängigen, Initialkonvergenz aus. Im Gegensatz zum bekannten RLS-Algorithmus (Recursive Least Squares), der eine numerische Komplexität von $\mathrm{O}\left(N^{2}\right)$ besitzt, steigt der Rechenaufwand beim SFTF linear mit der Koeffizientenanzahl $(\mathrm{O}(N))$.

Eine ausführliche Darstellung der verschiedenen adaptiven Algorithmen findet sich z.B. in [40] und [15]. Eine etwas andere, auf der geometrischen Betrachtung der Algorithmen basierende Übersicht gibt Alexander in [1].

\subsection{Streckenerkennung}

Im folgenden ist als einfachste Anwendung eines adaptiven Filters die sogenannte Streckenerkennung erläutert. Das Prinzip einer solchen Streckenerkennung zwischen einem Aktuator (AK) und einem stromab positionierten Sensor (S) ist in Abbildung 3.2 dargestellt. Als Eingangssignal für das digitale Filter und den Adaptionsalgorithmus dient ein im Computer generiertes Störsignal $x[n]$ (z.B. weißes Rauschen). Dieses Störsignal wird gleichzeitig als Anregungssignal für den Störaktuator verwendet. Nach Durchlaufen der Strecke $A(z)$ generiert das Anregungssignal $x[n]$ in der Grenzschicht eine Störwelle, die sich über die als linear angenommene physikalische Strecke $P(z)$ stromab zum Fehlersensor ausbreitet. Das dort aufgenommene Signal wird mit $B(z)$ gefiltert und liegt danach als desired-Signal $d[n]$ im Computer vor.

In den Übertragungsfunktionen $A(z)$ und $B(z)$ sind dabei folgende Elemente des Aufbaus zusammengefaßt:

- $A(z)$ : DA-Wandler, Rekonstruktionstiefpaß, Verstärker, DruckkammerLautsprecher, Aktuatorkammer und -schlitz sowie die Umwandlung der wandnormalen Fluidbewegung in eine Instabilitätswelle (Rezeptivität)

- $B(z)$ : Anemometer, Verstärker, Anti-Aliasing Filter, AD-Wandler

Sind alle Übertragungsfunktionen linear, so ergibt sich nach abgeschlossener Ad- 
aption als Übertragungsfunktion des adaptiven Filters

$$
W(z)=A(z) P(z) B(z)
$$

wodurch das Fehlersignal $e[n]$ minimal wird. Hierbei wurden alle Teilübertragungsstrecken und damit auch die Lage des Minimums der Kostenfunktion bezüglich der Filterkoeffizienten als zeitinvariant angenommen. Anhand der so erhaltenen Übertragungsfunktion können nun die Übertragungseigenschaften der physikalischen Strecke $P(z)$ untersucht werden, wobei allerdings die Teilstrecken $A(z)$ und $B(z)$ eventuell separat bestimmt und herausgerechnet werden müssen.

Eine zweite Konfiguration der Streckenerkennung, die in dieser Arbeit verwendet wurde, ist in Abbildung 3.3 dargestellt. Hier wird versucht, den Übertragungsprozeß zwischen zwei stromab gestaffelten Sensoren in der Grenzschicht nachzubilden. Als Filter-Übertragungsfunktion erhält man hier

$$
W(z)=P(z) \frac{B_{2}(z)}{B_{1}(z)}
$$

und somit im Fall gleicher Übertragungsfunktionen in den Signalaufnahmewegen $B_{1}(z)$ und $B_{2}(z)$ direkt die physikalische Strecke $P(z)$.

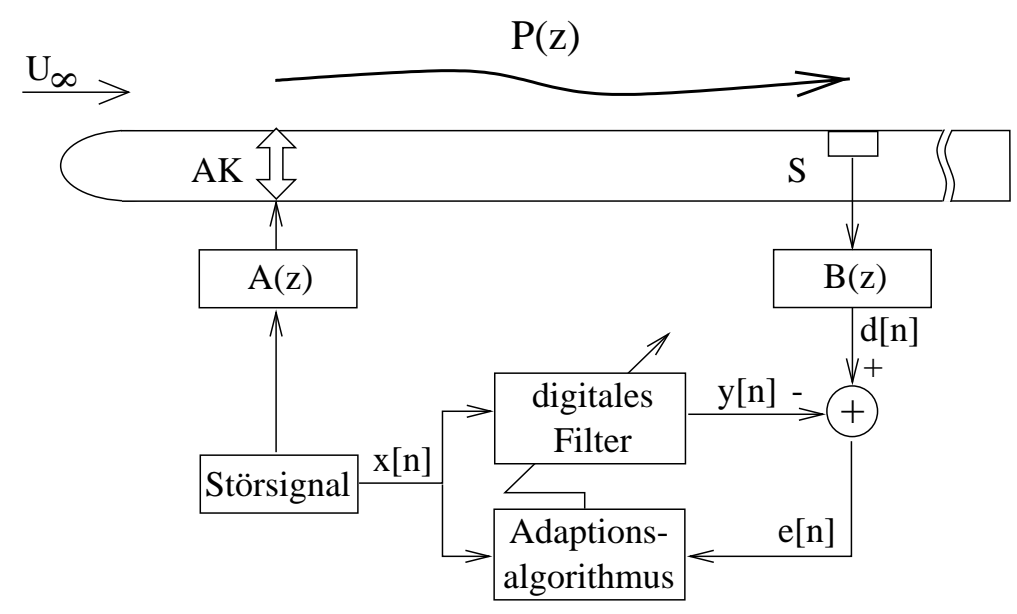

Abbildung 3.2: Prinzip der Streckenerkennung zwischen dem Störaktuator und einem Sensor

\subsection{Dynamische Stabilisierung}

Im folgenden wird das Prinzip der dynamischen Stabilisierung erläutert, wie sie in Abbildung 3.4 skizziert ist. Bei den hier vorgestellten Experimenten wurde die 


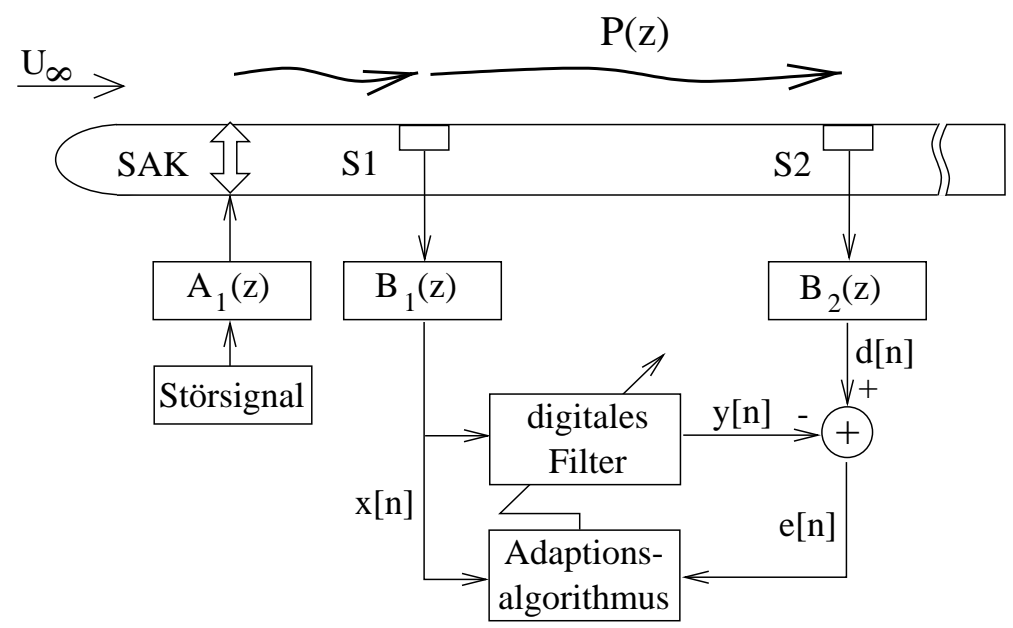

Abbildung 3.3: Prinzip der Streckenerkennung zwischen zwei Sensoren

Kompensation künstlich angeregter Störungen untersucht. Hierfür wird als Anregungssignal für den Störaktuator weißes Rauschen generiert, das nach Durchlaufen der Anregungs-Übertragungsfunktion $A_{1}(z)$ in der Grenzschicht Störwellen anregt. Diese werden,verzögert und spektral gefärbt durch die physikalische Strecke $P_{1}(z)$, stromab vom Referenzsensor (RS) aufgenommen. Während sich die Störung über die Strecke $P_{2}(z)$ weiter stromab ausbreitet, erzeugt das Referenzsignal nach der Aufnahme $\left(B_{1}(z)\right)$ und Filterung durch das digitale Filter und die Anregungs-Übertragungsfunktion $A_{2}(z)$ am Kompensationsaktuator eine weitere künstliche Störung.

Damit die mit dem Störaktuator und Kompensationsaktuator erzeugten Störwellen eine Phasenverschiebung von $\pi$ aufweisen (gegenphasig), wodurch sie sich durch destruktive Interferenz auslöschen, ist das digitale Filter $W(z)$ wie folgt einzustellen:

$$
W(z)=\frac{P_{2}(z)}{B_{1}(z) A_{2}(z)} .
$$

Hierbei ist zu beachten, daß das Filterausgangssignal mit -1 multipliziert wird. Die verbleibenden Störungen werden weiter stromab, außerhalb des Nahfeldes des Kompensationsaktuators, mit dem Fehlersensor (FS) aufgenommen. Da das Filterausgangssignal hier noch die Strecken $A_{2}(z), S^{\prime}(z)$ und $B_{2}(z)$ durchläuft, bevor es dem Adaptionsalgorithmus über das Fehlersignal $e[n]$ zur Verfügung steht muß das Referenzsignal $x[n]$ der Update-Vorschrift 3.25 noch mit einer Schätzung dieser Strecken (Sekundärstrecke) vorgefiltert werden. Man erhält so den sogenannten filtered-x LMS-Algorithmus (FXLMS)[19]. Eine anschauliche Ableitung dieses Algorithmus wird in Anhang A gegeben. Die Sekundärstreckenschätzung wurde hier jeweils vor der eigentlichen dynamischen Stabilisierung mittels einer 
Streckenerkennung zwischen dem Störaktuator und dem Fehlersensor bestimmt.

Die eingezeichnete Strecke $R^{\prime}(z)$ bezeichnet eine mögliche Rückkopplung vom Kompensationsaktuator zum Referenzsignal, die in Abhängigkeit von der Schleifenverstärkung und Phasenlage zu einer Instabilität des Systems führen kann und daher zum Beispiel rechnerisch anhand einer Rückkopplungsstreckenschätzung $\left(A_{2}(z) R^{\prime}(z) B_{1}(z)\right)$ aus dem Referenzsignal zu entfernen ist. Die Experimente haben jedoch gezeigt, daß hier die Ausbreitung entgegen der Anströmungsrichtung für die betrachteten Störwellen vernachlässigbar ist.

Damit nach diesem Schema eine Kompensation der Störungen möglich ist, müssen drei Voraussetzungen erfüllt sein. Zunächst müssen die zu kompensierenden Störungen beobachtbar, d.h. sowohl im Referenz- als auch im Fehlersignal enthalten sein. Desweiteren muß die Steuerbarkeit gewährleistet sein, das heißt der Kompensationsaktuator muß in der Lage sein, die jeweilige Störmode anzuregen und die Übertragungsfunktion $A_{2}(z)$ darf im Störfrequenzbereich keine Nullstellen aufweisen. Als letztes muß das System kausal sein, d.h. die Gruppenlaufzeit der physikalischen Strecke $P_{2}(z)$ darf nicht größer sein als die Summe der Gruppenlaufzeiten von $B_{1}(z)$ und $A_{2}(z)$ (Beeinflussungszweig), da sonst die Kompensationsstörung nicht „rechtzeitig“ generiert werden kann. Bei gegebenen Komponenten im Beeinflussungszweig ist durch die Kausalitätsforderung also ein einzuhaltender Minimalabstand zwischen dem Referenzsensor und dem Kompensationsaktuator gegeben.

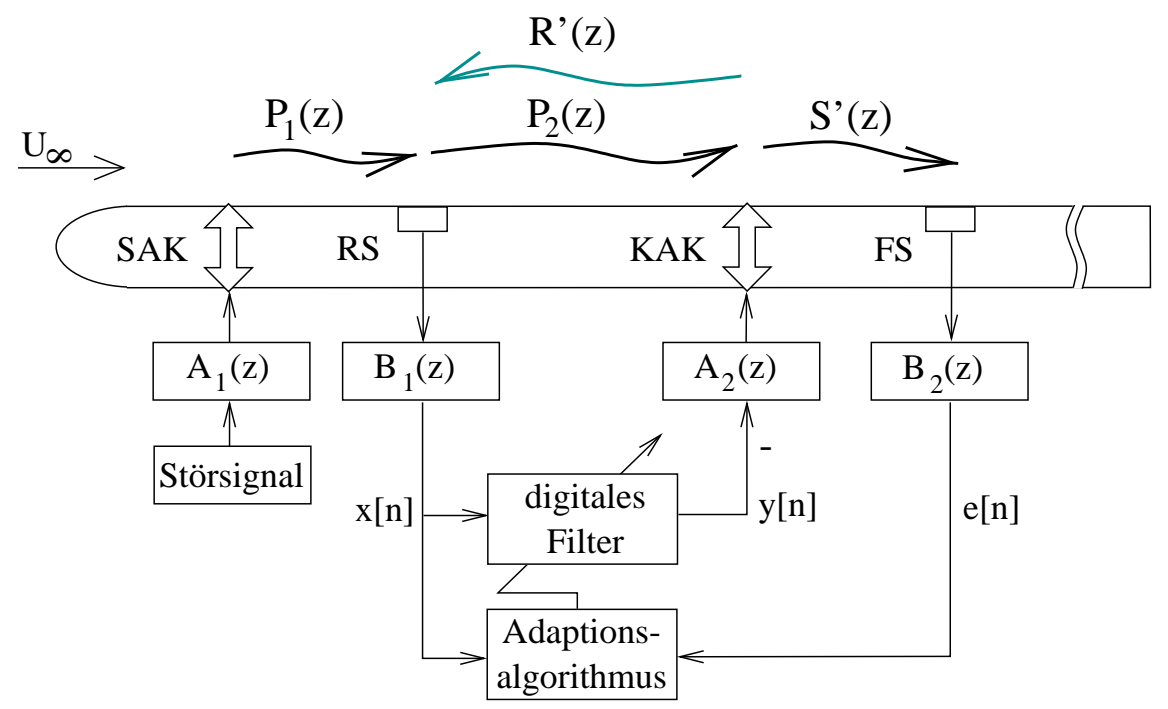

Abbildung 3.4: Prinzip der dynamischen Stabilisierung 


\subsection{Einfluß unkorrelierter Störkomponenten}

Bei den bisherigen Überlegungen wurde davon ausgegangen, daß die mit dem Referenz- und dem Fehlersensor aufgenommenen Signale miteinander vollständig linear korreliert sind, und somit über die ideale Nachbildung aller Teilstrecken durch das digitale Filter eine vollständige Störkompensation möglich ist. Bei realen Experimenten können jedoch Meßrauschen oder z.B. nichtlineare Prozesse in der Grenzschicht dazu führen, daß ein Teil der Signalleistung zwischen den beiden Sensoren nicht linear korreliert ist. In Abbildung 3.5 ist das Prinzip der dynamischen Stabilisierung als Streckenerkennungsproblem zwischen dem mit der Sekundärstrecke gefilterten Referenzsignal $x^{\prime}[n]$ und dem mit dem Fehlersensor aufgenommenen Signal - hier als desired-Signal $d[n]$ bezeichnet - dargestellt.Für Details dieser Umformung siehe Anhang A. Zusätzlich sind die Rauschterme $u[n]$ und $v[n]$ eingetragen, wobei vorausgesetzt wird, daß diese sowohl untereinander als auch mit dem Referenzsignal $x[n]$ unkorreliert sind. Die Strecke

$$
W_{\mathrm{OS}}^{\mathrm{o}}=\frac{P_{2}(z)}{B_{1}(z) A_{2}(z)}
$$

ist gleich der Wiener-Lösung ohne diese Störkomponenten.

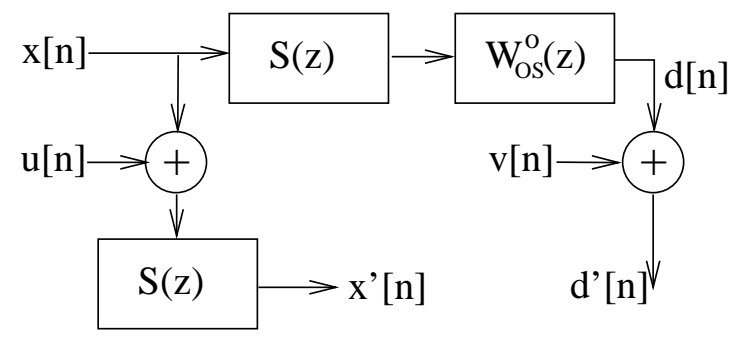

Abbildung 3.5: Einfluß unkorrelierter Störungen auf die dynamische Stabilisierung

Das optimale Filter mit diesen Störungen ergibt sich im Frequenzbereich über

$$
W^{o}(f)=\frac{S_{d^{\prime}, x^{\prime}}(f)}{S_{x^{\prime}, x^{\prime}}(f)} .
$$

Für die spektrale (Auto-)Leistungsdichte des Signals $x^{\prime}[n]$ erhält man

$$
\begin{aligned}
S_{x^{\prime}, x^{\prime}}(f) & =\lim _{M \rightarrow \infty} \frac{1}{M}\left\langle\mathcal{F}\left\{x_{M}^{\prime}[n]\right\} \mathcal{F}\left\{x_{M}^{\prime}[n]\right\}^{*}\right\rangle \\
& =\lim _{M \rightarrow \infty} \frac{1}{M}\left\langle[S(f) U(f)+S(f) X(f)][S(f) U(f)+S(f) X(f)]^{*}\right\rangle \\
& =|S(f)|^{2}\left(S_{u, u}+S_{x, x}\right),
\end{aligned}
$$


wobei $\mathcal{F}\left\{x_{M}[n]\right\}$ für die diskrete Fouriertransformation eines gefensterten Zeitsignals der Länge $M$ steht. Für die spektrale Kreuzleistungsdichte zwischen $x^{\prime}[n]$ und $d^{\prime}[n]$ ergibt sich

$$
\begin{aligned}
S_{d^{\prime}, x^{\prime}}(f) & =\lim _{M \rightarrow \infty} \frac{1}{M}\left\langle\mathcal{F}\left\{d_{M}^{\prime}[n]\right\} \mathcal{F}\left\{x_{M}^{\prime}[n]\right\}^{*}\right\rangle \\
& =\lim _{M \rightarrow \infty} \frac{1}{M}\left\langle\left[W_{\mathrm{OS}}^{\mathrm{o}}(f) S(f) X(f)+V(f)\right][S(f) U(f)+S(f) X(f)]^{*}\right\rangle \\
& =|S(f)|^{2} W_{\mathrm{OS}}^{\mathrm{o}}(f) S_{x, x},
\end{aligned}
$$

und die Übertragungsfunktion des optimalen Filters ist somit

$$
\begin{aligned}
W^{o}(f) & =\frac{W_{\mathrm{OS}}^{\mathrm{o}}(f) S_{x, x}(f)}{S_{x, x}(f)+S_{u, u}(f)} \\
& =\frac{W_{\mathrm{OS}}^{\mathrm{o}}(f)}{1+1 / \rho(f)},
\end{aligned}
$$

wobei $\rho=S_{x, x}(f) / S_{u, u}(f)$ das Signal-Rausch-Verhältnis am Referenzsensor bezeichnet. Der unkorrelierte Störterm $u[n]$ führt also, im Vergleich mit der ungestörten Lösung, zu einer Veränderung des Betrages, nicht jedoch der Phase der erkannten Übertragungsfunktion. Dies ist leicht einzusehen, da die Übertragungsfunktion hier ein Kompromiß zwischen der unerwünschten Übertragung des unkorrelierten Signalanteils $u[n]$ und der möglichst guten Nachbildung der linearen Übertragungsprozesse sein muß. Für die Anwendung ist nun interessant, welches Fehlersignal sich mit dem optimalen Filter aus Gl. 3.35 ergibt. Die diskrete Fouriertransformation des Fehlers $e[n]=d^{\prime}[n]-\mathbf{x}^{\prime T}[n] \mathbf{w}^{\mathrm{o}}$ liefert

$$
\begin{aligned}
\mathcal{F}\{e[n]\} & =S(f)\left[W_{\mathrm{OS}}^{\mathrm{o}}(f) X(f)-W^{o}(f)(X(f)+U(f))\right]+V(f) \\
& =S(f) W_{O S}^{o}(f)\left[X(f)-\frac{S_{x, x}}{S_{x, x}+S_{u, u}}(X(f)+U(f))\right]+V(f) \\
& =S(f) W_{\mathrm{OS}}^{\mathrm{o}}(f)\left[\frac{S_{u, u}(f) X(f)-S_{x, x}(f) U(f)}{S_{x, x}(f)+S_{u, u}(f)}\right]+V(f) \\
& =S(f) W_{\mathrm{OS}}^{\mathrm{o}}(f)\left[\frac{X(f)}{1+\rho(f)}-\frac{U(f)}{1+1 / \rho(f)}\right]+V(f) .
\end{aligned}
$$

Als erstes zeigt sich, daß die Störkomponente $v[n]$ vollständig im Fehlerspektrum enthalten ist, dies war auch nicht anders zu erwarten, da diese Störung als unkorreliert mit dem Referenzsignal und damit auch mit dem desired-Signal angenommen wurde und somit nicht kompensiert werden kann. Desweiteren finden sich mit dem Signal-Rausch-Abstand gewichtete Anteile sowohl des unverrauschten Referenzsignals $x[n]$ als auch des Rauschterms $u[n]$. Für die spektrale Leistungs- 
dichte des Fehlersignals erhält man

$$
\begin{aligned}
S_{e, e}(f) & =\lim _{M \rightarrow \infty} \frac{1}{M}\left\langle\mathcal{F}\left\{e_{M}[n]\right\} \mathcal{F}\left\{e_{M}[n]\right\}^{*}\right\rangle \\
& =|S(f)|^{2}\left|W_{\mathrm{OS}}^{\mathrm{o}}(f)\right|^{2}\left[\frac{S_{u, u}^{2}(f) S_{x, x}(f)+S_{x, x}^{2}(f) S_{u, u}(f)}{\left(S_{x, x}(f)+S_{u, u}(f)\right)^{2}}\right]+S_{v, v}(f) \\
& =|S(f)|^{2}\left|W_{\mathrm{OS}}^{\mathrm{o}}(f)\right|^{2} \frac{S_{x, x}(f)}{1+\rho(f)}+S_{v, v}(f) \\
& =\frac{S_{d, d}(f)}{1+\rho(f)}+S_{v, v}(f)
\end{aligned}
$$

Neben der unkorrelierten Leistung $S_{v, v}(f)$ kann somit in Abhängigkeit vom Signal-Rausch-Abstand am Referenzsensor $\rho(f)$ auch ein Teil der linear korrelierten Signalleistung $S_{d, d}(f)$ nicht kompensiert werden. Dieses Ergebnis zeigt deutlich, wie wichtig die meßtechnische Optimierung des Signal-Rausch-Abstandes bei der Aufnahme des Referenzsignales ist.

Für die Praxis gibt es einen einfachen Weg, die Fehlerleistung nach abgeschlossener Adaption eines linearen adaptiven Filters und damit den möglichen Kompensationserfolg anhand der Kohärenz zwischen dem Referenzsignal (hier $x^{\prime}[n]$ ) und dem Signal des Fehlersensors (hier $d^{\prime}[n]$ ) vor dem eigentlichen Experiment abzuschätzen. Mit dem Betragsquadrat der Kohärenz $\gamma_{d^{\prime}, x^{\prime}}(f)$

$$
C_{d^{\prime}, x^{\prime}}(f)=\left|\gamma_{d^{\prime}, x^{\prime}}(f)\right|^{2}=\frac{\left|S_{d^{\prime}, x^{\prime}}(f)\right|^{2}}{S_{d^{\prime}, d^{\prime}}(f) S_{x^{\prime}, x^{\prime}}(f)}
$$

ergibt sich für den Restfehler einer linearen Nachbildung

$$
S_{e, e}(f)=\left[1-C_{d^{\prime}, x^{\prime}}(f)\right] S_{d^{\prime}, d^{\prime}}(f),
$$

wobei der Anteil $C_{d^{\prime}, x^{\prime}}(f) \cdot S_{d^{\prime}, d^{\prime}}(f)$ gerade die zu $x^{\prime}[n]$ linear korrelierte (kohärente) spektrale Leistungsdichte beschreibt.

Setzt man diesen Fehler ins Verhältnis zur vorhandenen Störleistung $S_{d^{\prime}, d^{\prime}}(f)$, so ergibt sich als mögliche Fehlerreduktion infolge einer linearen dynamischen Stabilisierung

$$
-10 \log _{10}\left[1-C_{d^{\prime}, x^{\prime}}(f)\right]
$$

\subsection{Kompensation von elektrischen Einstreuun- gen}

Einstreuungen bei der Netzfrequenz und ihren Harmonischen in die Anemometersignale stellten bei dem verwendeten Aufbau ein großes Problem dar. Da verschiedene Veränderungen auf der analogen Seite der Meßtechnik keine wesentlichen 
Verbesserungen erbrachten, wurden diese Brummkomponenten mittels adaptiver Filter aus den Meßsignalen entfernt.

Dabei wird aus der mit einem Transformator aufgenommenen Netzspannung ein unsymmetrisches Rechtecksignal mit der Grundfrequenz von $50 \mathrm{~Hz}$ und allen relevanten Höherharmonischen gewonnen. Dieses Signal dient als Eingangssignal $x[n]$ für das adaptive Filter. Als desired-Signal $d[n]$ wird das jeweilige Meßsignal verwendet. Das sich ergebende Fehlersignal $e[n]$ wird nun vom Algorithmus mit dem Rechtecksignal dekorreliert, so daß sich als Fehlersignal das Meßsignal ohne störendes Netzbrummen ergibt. Der Erfolg einer solchen Brummkompensation ist in Abb. 3.6 anhand des Leistungsspektrums eines mit dem Referenzsensor aufgenommenen Signals ohne und mit Brummkompensation dargestellt.

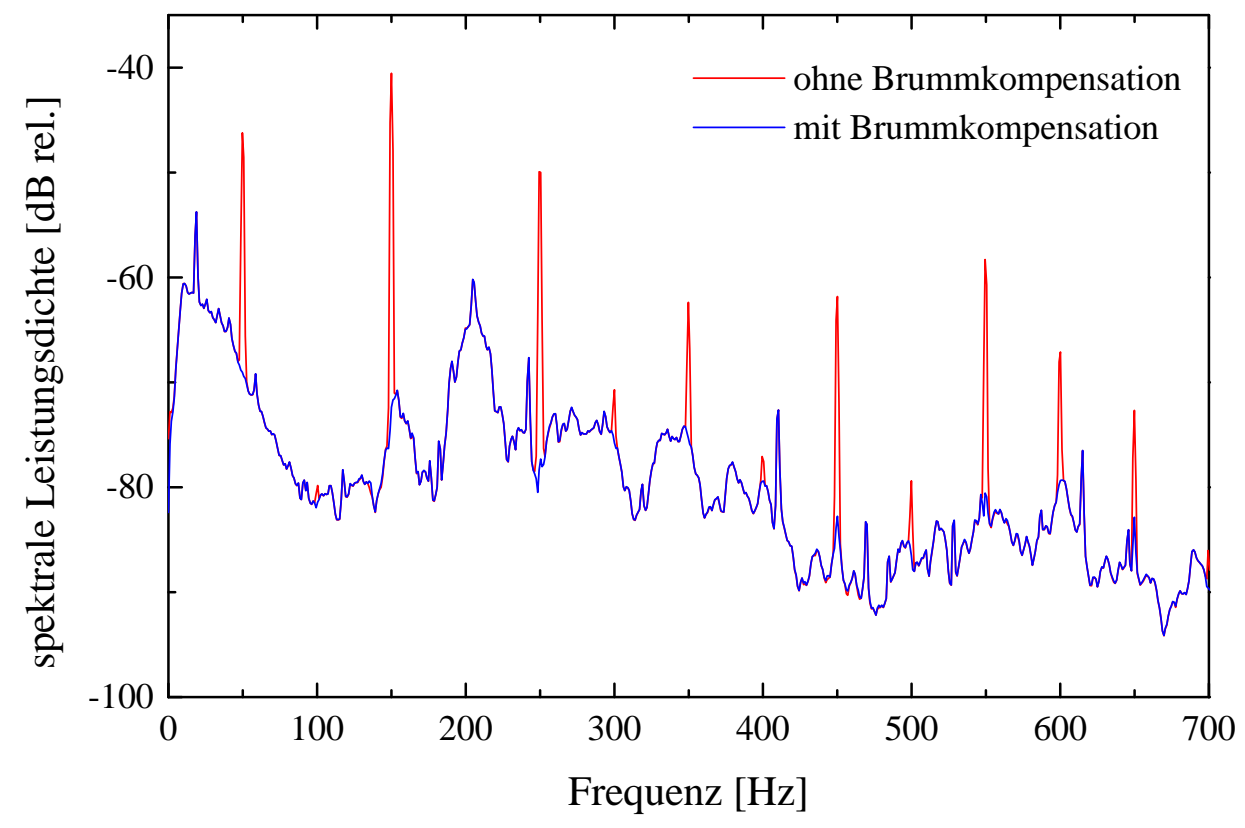

Abbildung 3.6: Spektrale Leistungsdichte des Sensorsignals ohne und mit Brummkompensation (Referenzsensor ohne Störanregung)

Es zeigt sich eine nahezu vollständige Kompensation der Brummkomponenten.

Es ist noch anzumerken, daß die gezeigte Brummkompensation keine rein kosmetische Korrektur der Meßsignale darstellt. Neben dem ungünstigen Einfluß solcher Störungen auf die Konvergenz der Adaptionsalgorithmen können sie bei der dynamischen Stabilisierung noch zu zusätzlichen unerwünschten Effekten führen. Sind solche Störungen sowohl im Referenzsignal als auch im Fehlersignal enthalten, bilden sich bei den entsprechenden Frequenzen Korrelationen, die jedoch nicht durch eine Störausbreitung in der Grenzschicht bedingt sind (Nebenwegsstörungen). Dies kann dazu führen, daß der Kompensationsaktuator TS-Wellen bei den jeweiligen Frequenzen generiert, die zwar zu einer Reduktion der Nebenwegsstörungen 
am Fehlersensor führen, sich aber danach quasi unbemerkt in der Grenzschicht ausbreiten. Im Hinblick auf die spätere Anwendung wäre daher die mögliche Entstehung solcher Nebenwegsstörungen z.B. aufgrund von Sensorschwingungen zu berücksichtigen.

\subsection{Geräte und Programme zur Signalverarbei- tung}

Für die ersten Untersuchungen wurden die adaptiven Algorithmen auf einem sogenannten Evaluation Board (ADSP-2106x) der Firma Analog Devices implementiert. Im Gegensatz zu diesen speziellen Signalprozessoren bieten herkömmliche Personal Computer (PC) bei vergleichsweise geringen Kosten mittlerweile eine enorme Rechenleistung. Aus diesem Grund wurde von Herrn Dipl.-Phys. Gero Timann (Drittes Physikalisches Institut) ein Programm entwickelt, das es ermöglicht, die digitale Signalverarbeitung auf einem PC in Echtzeit durchzuführen (PPDSP, Pentium Pro Digital Signal Processing). Durch die integrierte graphische Oberfläche ist es möglich, sich während der Messung zum Beispiel die Impulsantworten der Filter oder ihre Übertragungsfunktionen anzusehen. Desweiteren können Parameter während der Messung angepaßt und z.B. Filtervektoren jederzeit abgespeichert werden.

Für die AD/DA-Wandlung (Analog-Digital/Digital-Analog) wurde ein Entwicklungssystem (EVM56002) der Firma Motorola eingesetzt. Die Entwicklungsplatine beinhaltet neben dem DSP56002 Signalprozessor auch einen 16-Bit CodecWandler (CS4215; Chrystal) mit zwei AD/DA-Kanälen. Durch die Ansteuerung eines zweiten Wandlerbausteins vom selben Typ konnte das System auf vier AD/DA-Kanäle erweitert werden. Jeweils zwei dieser Systeme kommunizieren über eine ISA-Schnittstellenkarte [8] mit dem PC. Auf diese Weise wurden Wandlerbausteine mit bis zu 16-Kanälen aufgebaut, welche über das entwickelte Programm (PPDSP) angesprochen werden können.

Die adaptiven Filteralgorithmen wurden aufgeteilt in optimierte Assemblerroutinen für Vektoroperationen, die bei den unterschiedlichen Filteralgorithmen immer wieder benötigt werden und einen großen Anteil der Rechenzeit ausmachen (Skalarprodukt, Vektorgradient), und den Rest, der in C programmiert wurde. Dieses Verfahren führt zu sehr effizienten Algorithmen, die trotzdem leicht zu programmieren sind. Die angesprochenen Vektoroperationen (SDOT, SAXPY etc.) gehören zum Standard der sog. BLAS1-library. Informationen zu optimierten Versionen dieser Bibliotheken finden sich z.B. in [18] oder auf der Intel-homepage (http://www.intel.com, math kernel library).

Es existiert somit ein sehr leistungsfähiges und komfortabel zu bedienendes Experimentiersystem, das zudem deutlich preisgünstiger ist, als kommerzielle auf 
DSP-Prozessoren basierende Lösungen.

Hinsichtlich der Kausalitätsanforderung ist ein Problem bei der Verwendung der Codec-Wandler die hohe Gruppenlaufzeit der internen digitalen Filter von 38 Zeitschritten für eine $\mathrm{AD} / \mathrm{DA}$-Wandlung. Benutzt man eine sehr hohe Abtastrate $f_{s}$, so ist zwar die Gruppenlaufzeit gemessen in [ms] geringer, jedoch wird so auch die verfügbare Rechenzeit zwischen zwei Abtastpunkten verringert. Daher wurde mit einem Abtastratenwechsel (Dezimator, Interpolator [29]) gearbeitet, wobei die Abtastrate der Codec-Wandler 10-mal so hoch war, wie die Datenrate für die eigentliche Signalverarbeitung. Durch geringere Anforderungen an die bei der Dezimation $\left(f_{s} \downarrow f_{s} / 10\right)$ und Interpolation $\left(f_{s} / 10 \uparrow f_{s}\right)$ notwendigen Tiefpaßfilter konnte insgesamt eine Reduktion der Gruppenlaufzeit auf etwa 8 Zeitschritte erreicht werden. Die zusätzlichen Tiefpaßfilter wurden auf dem DSP56002-Signalprozessor implementiert. Für einen Vergleich der Übertragungseigenschaften der AD/DA-Wandlung wurde eine Systemerkennung mit Abtastratenwechsel von $20.35 \mathrm{KHz}$ (Codec) auf $2.035 \mathrm{KHz}$ (intern) und eine Systemerkennung mit einer Abtastrate von $2.035 \mathrm{KHz}$ (Codec) durchgeführt. Aus- und Eingang des Wandlers wurden hierbei direkt verbunden. Aus den Impulsantworten wurden mittels der Fouriertransformation die Übertragungsfunktionen $H(f)$ berechnet. Die Gruppenlaufzeit ergibt sich aus dem Phasengang $\arg [H(f)]$ über

$$
\tau_{\mathrm{gr}}(f)=-\frac{1}{2 \pi} \frac{d}{d f}(\arg [H(f)]) .
$$

Abbildung 3.7 zeigt die durch den Abtastratenwechsel erzielte deutliche Reduktion der Gruppenlaufzeit und anhand der Übertragungsfunktionen (Abb. 3.8) läßt sich gut der Einfluß der zusätzlichen Tiefpaßfilter mit einer Grenzfrequenz von etwa $700 \mathrm{~Hz}$ erkennen. 


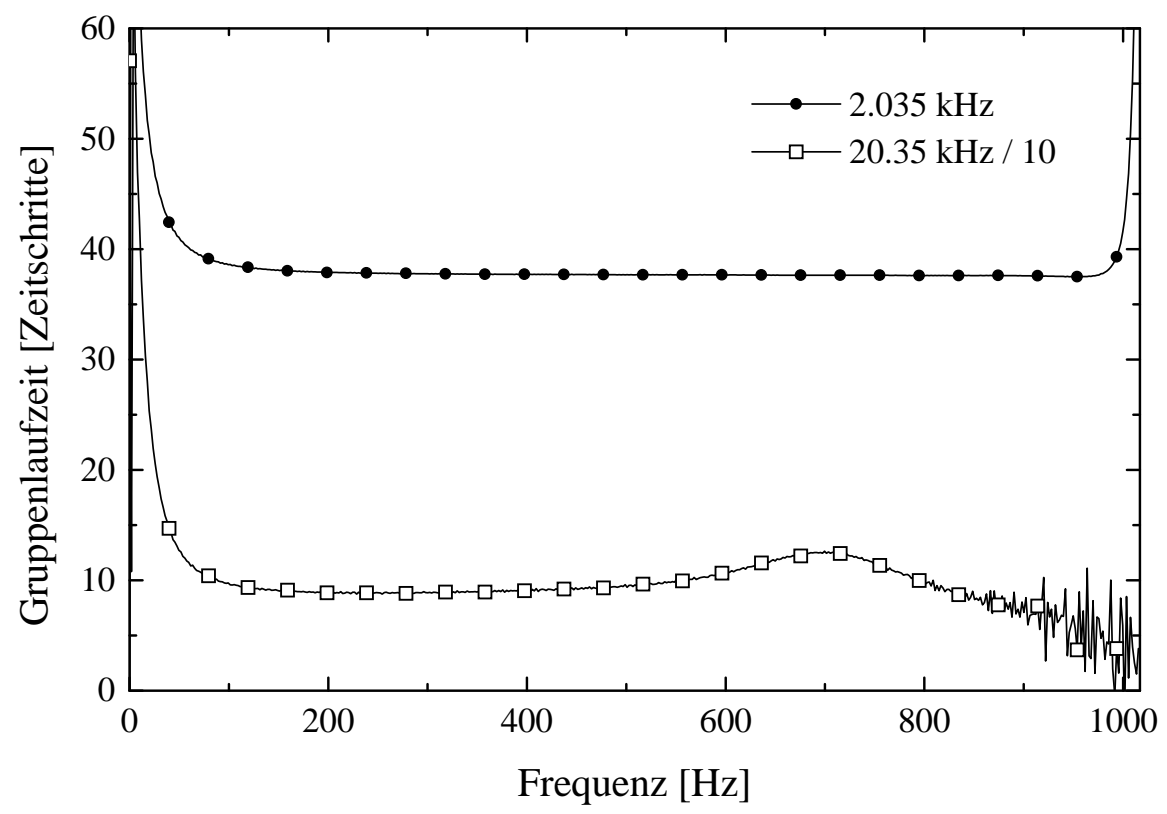

Abbildung 3.7: Gruppenlaufzeit der AD/DA-Wandlung

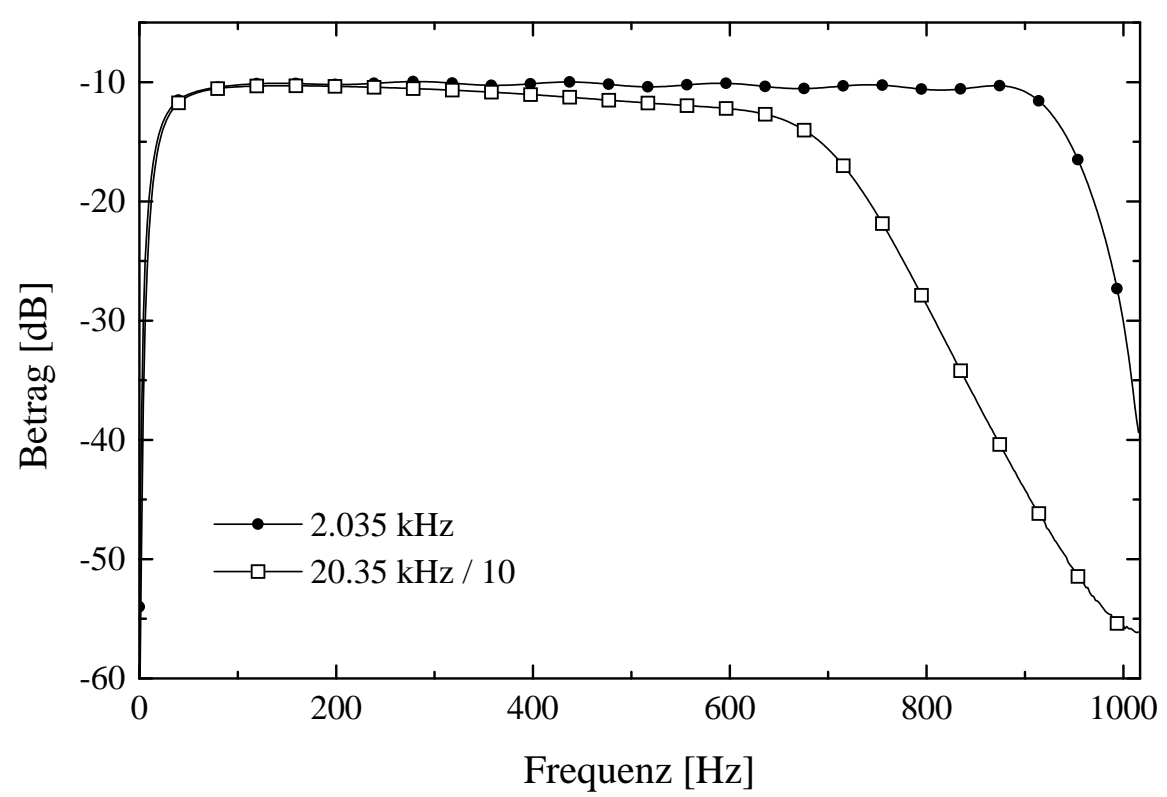

Abbildung 3.8: Frequenzgang der AD/DA-Wandlung 


\section{Kapitel 4}

\section{Stromabausbreitung linear korrelierter Grenzschichtstörungen}

Um einen Eindruck von den Ausbreitungsprozessen zwischen dem Störaktuator (SAK) und stromab liegenden Positionen zu erhalten, wurden als erstes Streckenerkennungsexperimente mit der in Abb. 3.2 dargestellten Konfiguration durchgeführt. Für den Leistungsverstärker zum Betrieb des Druckkammerlautsprechers (SAK) wurde zu Beginn der in dieser Arbeit dargestellten Experimente eine feste Einstellung gewählt und die Amplitudenänderung durch Variation der Amplitude des im Computer generierten Rauschens im Bereich $0-1$ (lineare Skala) vorgenommen. Somit sind alle Experimente hinsichtlich der Störamplitude direkt vergleichbar. Hier betrug die Amplitude 0,2. Bringt man für eine erste Messung den Hitzdraht direkt über den Aktuatorschlitz, so werden im wesentlichen die Übertragungsfunktionen $A(z)$ und $B(z)$ nachgebildet (s. Abb. 3.2). Schaltet man diese bei der Streckenerkennung für verschiedene Stromab-Positionen des Hitzdrahtes vor das eigentliche adaptive Filter, so wird mit diesem nur noch der eigentliche Ausbreitungsprozess $P(z)$ in der Grenzschicht erkannt. Von der Position direkt über dem Aktuator (Nahfeld) wurde der Hitzdraht nun schrittweise $(\Delta x=1 \mathrm{~cm})$ stromab verfahren, wobei jedesmal die physikalische Strecke $P(z)$ mit dem Filter nachgebildet wurde. Die sich einstellenden Filterkoeffizienten sind in Abb. 4.1 dargestellt. Da die Folge der Koeffizienten eines FIR-Filters gerade seiner Impulsantwort entspricht, sieht man an diesem Bild, wie sich ein Impuls, welcher an der Position des Störaktuators in die Grenzschicht eingebracht wird, stromab ausbreitet. Vergleicht man die Ausbreitung der Maxima und Minima mit der Ausbreitung der Einhüllenden des Wellenpaketes, so wird deutlich, daß die Phasengeschwindigkeit kleiner ist als die Gruppengeschwindigkeit.

Für einen Abstand von $18 \mathrm{~cm}$ zwischen dem Störaktuator und der Hitzdrahtson- 


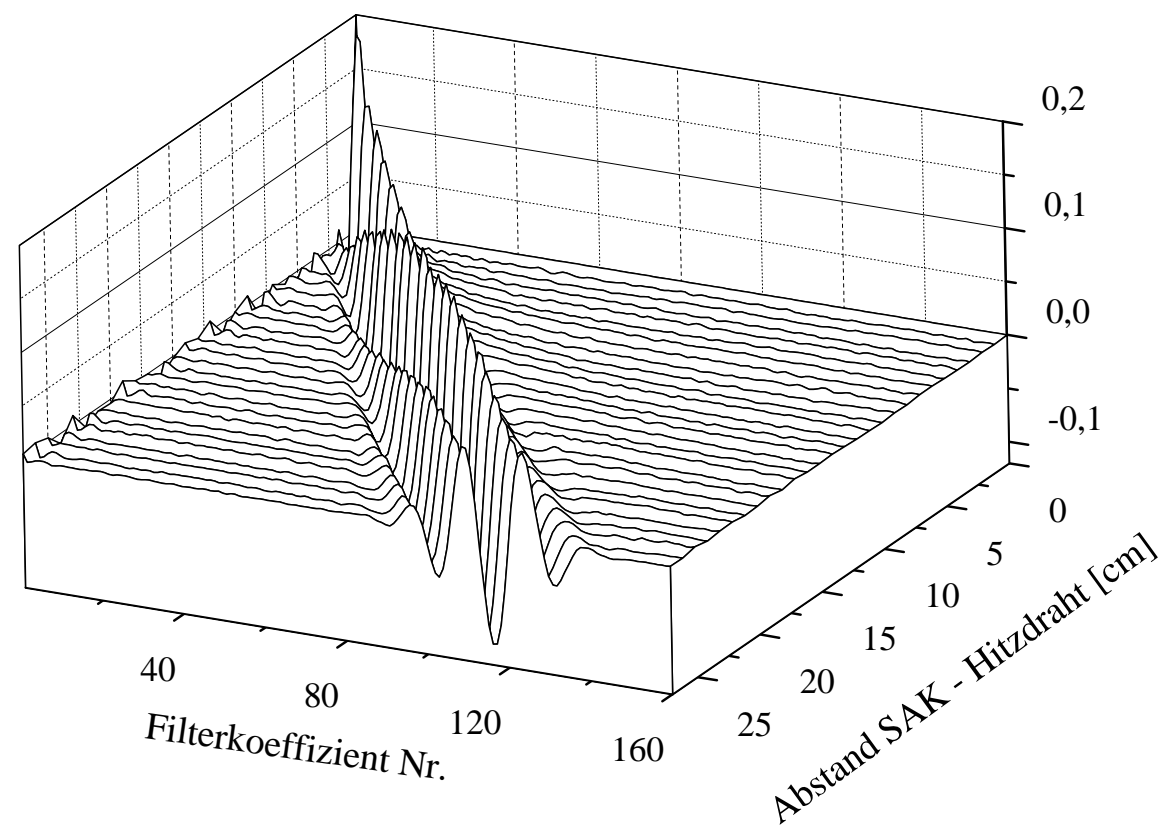

Abbildung 4.1: Streckenerkennung zwischen Störaktuator und Hitzdraht (Filterkoeffizienten)

de wurde aus der Impulsantwort die Übertragungsfunktion $H(f)$ berechnet. Ungefähr an dieser x-Position befindet sich der Aktuator (Nr. 13 des Arrays), der bei den Experimenten zur dynamischen Stabilisierung als Kompensationsaktuator (KAK) verwendet wurde. Aus dem Phasengang $\arg [H(f)]$ ) ergibt sich mit Gl. 3.41 die Gruppenlaufzeit und über,

$$
\tau_{\mathrm{ph}}(f)=-\frac{1}{2 \pi} \frac{1}{f}(\arg [H(f)])
$$

die Phasenlaufzeit bzw. Phasenverzögerung. Aus diesen Laufzeiten wurden mit dem Abstand $\Delta x=18 \mathrm{~cm}$ und der Samplingfrequenz $f_{s}=2035 \mathrm{~Hz}$ die Gruppenund die Phasengeschwindigkeit berechnet und in Abbildung 4.2, bezogen auf die Außengeschwindigkeit $U_{\infty}=11 \mathrm{~m} / \mathrm{s}$, aufgetragen. Die Gruppengeschwindigkeit liegt im Bereich von $c_{g r}=0,35 \cdots 0,42$ und steigt mit zunehmender Frequenz an. Die Phasengeschwindigkeit ist mit etwa $c_{p h}=0,3 \cdots 0,38$ wie schon anhand von Abb. 4.1 erläutert wurde, deutlich geringer. Für die schnellsten korrelierten Anteile beträgt die Gruppenlaufzeit vom Referenzsensor bis zum Störaktuator somit etwa $20 \mathrm{~ms}$ entsprechend 40 Zeitschritten bei einer Abtastrate von $f_{s}=$ $2 \mathrm{KHz}$.

Betrachtet man den Betrag der Fouriertransformierten der Impulsantworten aus Abbildung 4.1, so erhält man das Übertragungsverhalten im Frequenzbereich (siehe Abb. 4.3). Für die Position direkt über dem Störaktuator ist der Betrag 


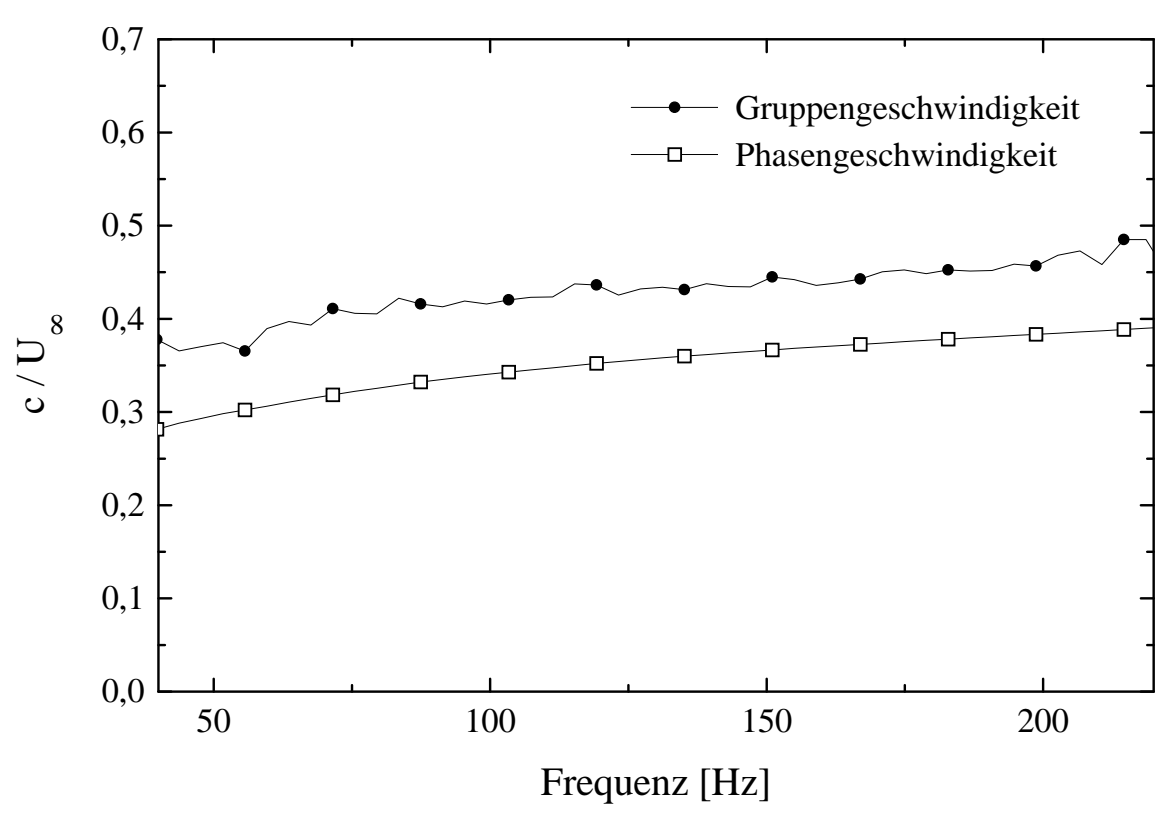

Abbildung 4.2: Ausbreitungsgeschwindigkeit linear korrelierter Störungen

durch die Vorfilterung per definitionem 1 entsprechend $0 \mathrm{~dB}$. Mit zunehmendem Abstand werden jedoch gerade hohe und tiefe Frequenzen gedämpft, und es ergibt sich die typische schmalbandige Übertragungsfunktion mit einer Anfachung der TS-Wellen stromab, wobei sich das Maximum zu tieferen Frequenzen hin verschiebt.

Abbildung 4.4 zeigt die Indifferenzkurve einer ebenen Grenzschicht für zweidimensionale Störungen, wie sie sich als Lösung der Orr-Sommerfeld-Gleichung ergibt (paralleles, lokales Eigenwertproblem [16]). Während Störungen mit Frequenzen, die sich bei einer bestimmten Reynoldszahl innerhalb der Indifferenzkurve befinden, angefacht werden, erfahren Störungen, deren Frequenz außerhalb der Kurve liegt, nach linearer Theorie eine Dämpfung. Für einen einfacheren Vergleich mit den experimentellen Ergebnissen wurden die Positionen des Störaktuators (SAK), des verwendeten Kompensationsaktuators (KAK), sowie des Referenzsensors (RS) und des Fehlersensors (FS) zusammen mit einigen nicht normierten Grenzfrequenzen eingetragen. In Tabelle 4.1 sind die wichtigsten Kennzahlen für alle Sensor- und Aktuatorpositionen zusammengefaßt. Der Frequenzbereich instabiler Störungen erstreckt sich dabei jeweils von $F, f_{\min }$ bis $F, f_{\max }$. 


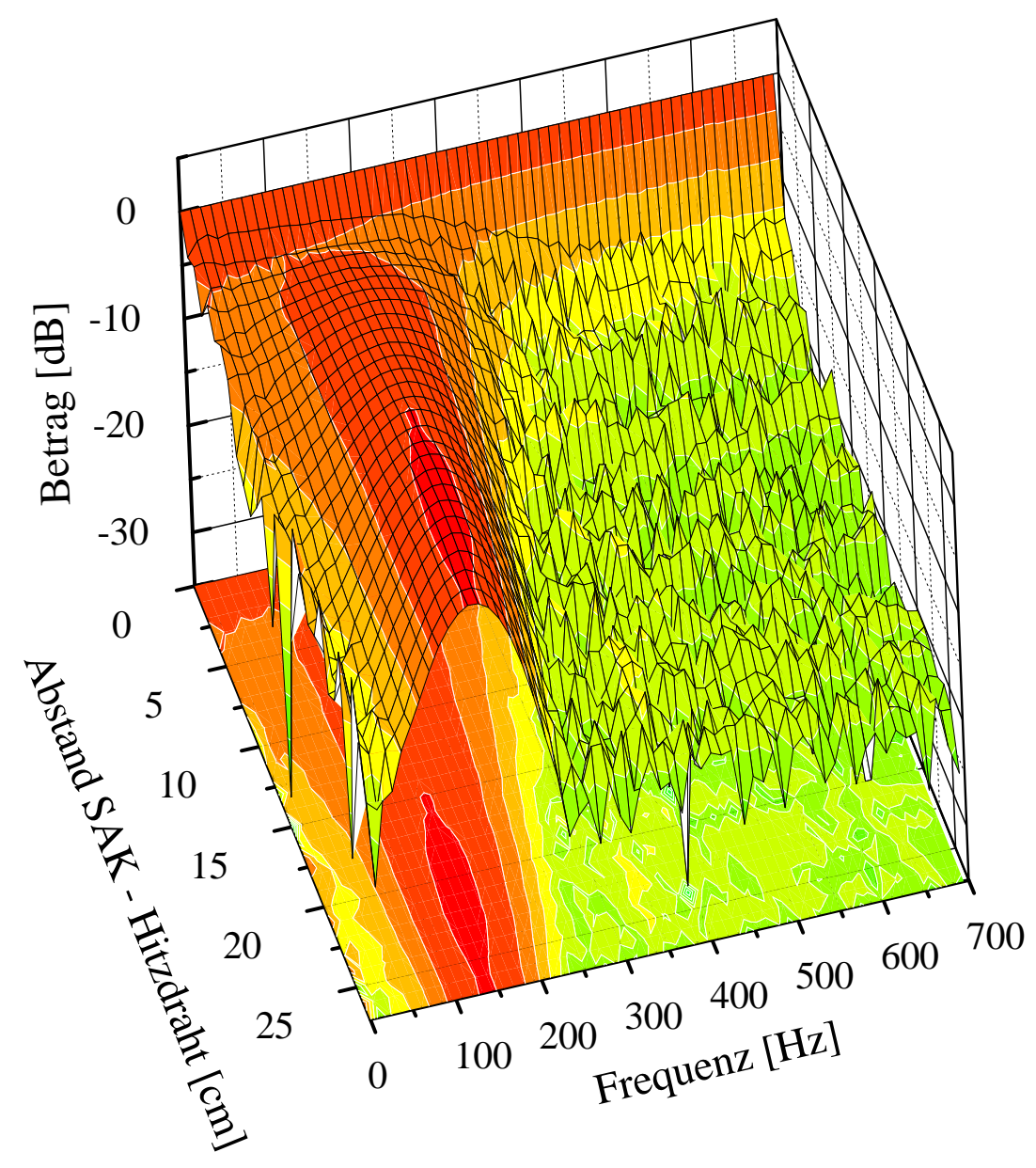

Abbildung 4.3: Streckenerkennung zwischen Störaktuator und Hitzdraht (Betrag der Übertragungsfunktion), Amplitude: 0,2

\begin{tabular}{|c||c|c|c|c|c|c|c|}
\hline & $x[\mathrm{~cm}]$ & $\mathrm{Re}_{\mathrm{x}}\left[10^{5}\right]$ & $\sqrt{\mathrm{Re}_{\mathrm{x}}}$ & $\mathrm{F}_{\text {min. }}$ & $\mathrm{F}_{\max .}$ & $\mathrm{f}_{\min .}[\mathrm{Hz}]$ & $\mathrm{f}_{\max .}[\mathrm{Hz}]$ \\
\hline \hline SAK & 18 & 1,09 & 332 & 168 & 238 & 211 & 299 \\
\hline RS & 26 & 1,67 & 409 & 107 & 196 & 135 & 247 \\
\hline KAK & 35,5 & 2,35 & 485 & 77 & 162 & 97 & 204 \\
\hline FS & 42 & 2,82 & 531 & 65 & 145 & 81 & 182 \\
\hline
\end{tabular}

Tabelle 4.1: Virtuelle Lauflängen, Reynoldszahlen und Grenzfrequenzen des angefachten Bereiches an den Sensor- und Aktuatorpositionen, $U_{\infty}=11 \frac{\mathrm{m}}{\mathrm{s}}$ 


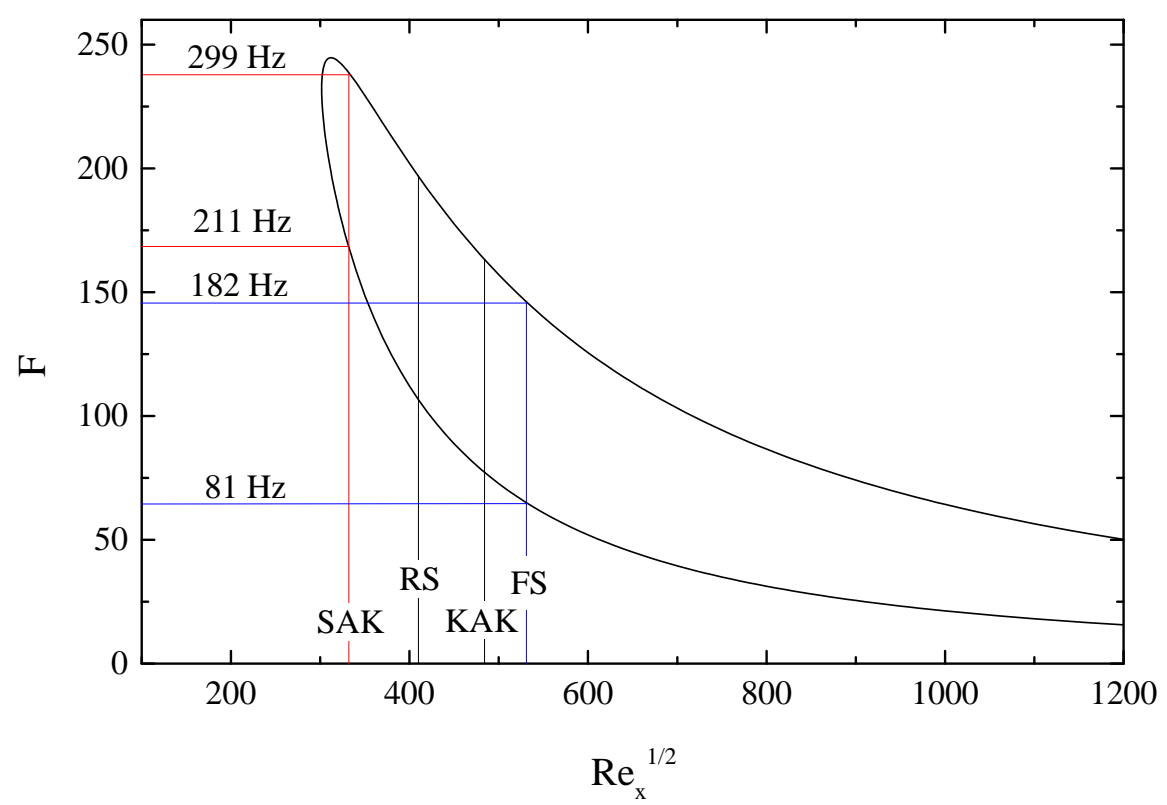

Abbildung 4.4: Indifferenzkurve einer ebenen Grenzschicht (Blasiusgrenzschicht) für zweidimensionale Störungen. Reynoldszahl bezogen auf die virtuelle Lauflänge $\operatorname{Re}_{\mathrm{x}}=\mathrm{U}_{\infty} \mathrm{x}_{\mathrm{V}} / \nu$, entdimensionalisierte Frequenz $F=\left(2 \pi f \nu / U_{\infty}^{2}\right) \cdot 10^{6}$ 


\section{Kapitel 5}

\section{Dynamische Stabilisierung (lineares Modell)}

In diesem Kapitel werden die Ergebnisse von Experimenten zur dynamischen Stabilisierung vorgestellt, bei denen als Modell zur Nachbildung der physikalischen Übertragungsfunktion in der Grenzschicht ein lineares FIR-Filter verwendet wurde. Insbesondere wurde hierbei die Kompensierbarkeit von künstlich eingebrachten zweidimensionalen Störungen in Abhängigkeit von ihrer Amplitude am Störaktuator untersucht, um mögliche Grenzen des linearen Modells beschreiben zu können.

\subsection{Versuchsergebnisse bei unterschiedlichen Störamplituden}

Im folgenden sind die Ergebnisse von vier Kompensationsexperimenten mit unterschiedlichen Störamplituden dargestellt. Alle anderen Versuchsparameter wurden konstant gehalten. Als Kompensationsaktuator (KAK) wurde Aktuator Nr. 13 des Arrays gewählt. Hinsichtlich der geometrischen Parameter und Reynoldszahlen siehe Tabelle 4.1 in Kapitel 4. Um zu überprüfen, inwieweit der Abstand zwischen dem Referenzsensor und dem Störaktuator der Kausalitätsanforderung genügt, wurde vor den Experimenten zur dynamischen Stabilisierung die Gruppenlaufzeit des Beeinflussungszweiges, also der Strecken $B_{1}(z)$ und $A_{2}(z)$ (Abb. 3.4) anhand der Ergebnisse einer Streckenerkennung zwischen dem Störaktuator und einem direkt darüber positionierten Hitzdrahtsensor bestimmt. Diese Abschätzung ist so nur möglich, da sich die Übertragungseigenschaften des Hitzdrahtsensors und des bei der dynamischen Stabilisierung verwendeten Oberflächenhitzdrahtes (Referenzsensor) kaum unterschieden. 


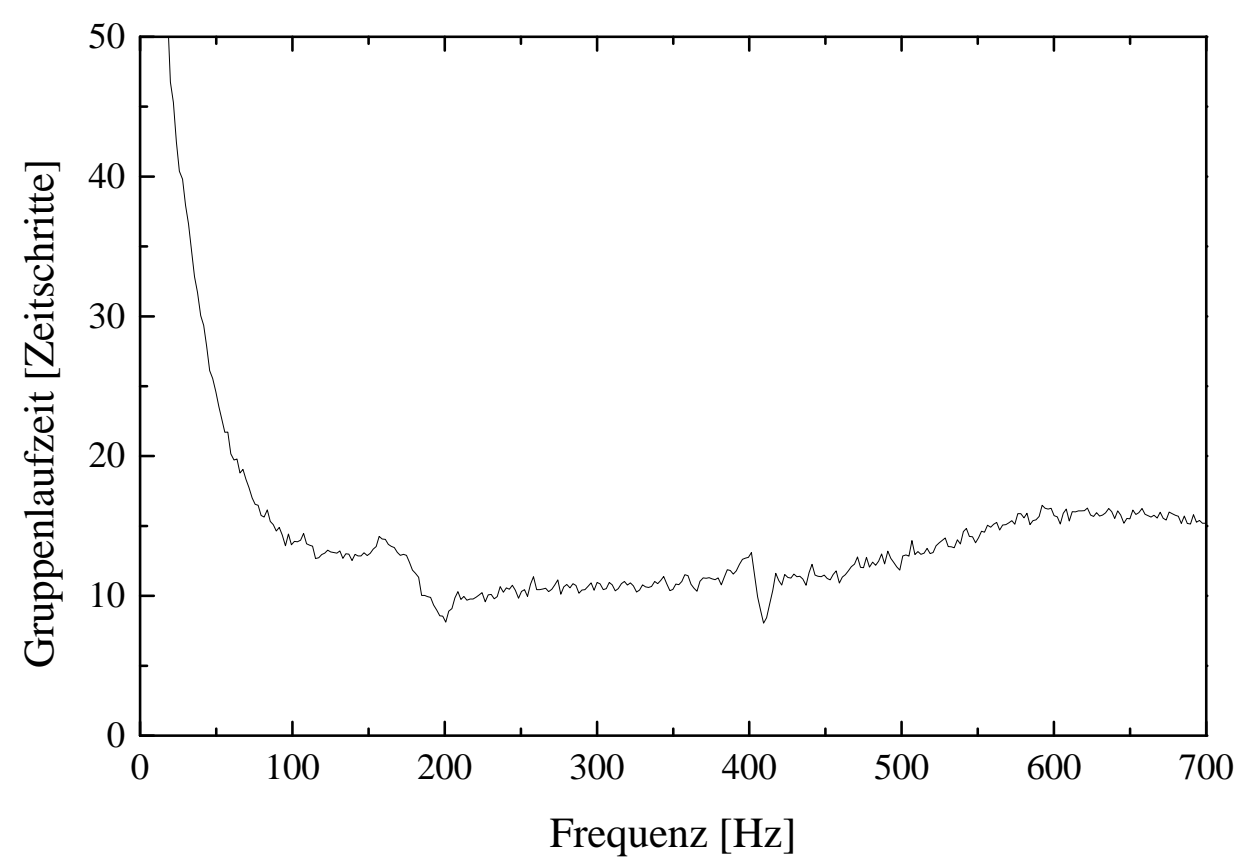

Abbildung 5.1: Gruppenlaufzeit des Beeinflussungszweiges

Im Frequenzbereich zwischen $50 \mathrm{~Hz}$ und $200 \mathrm{~Hz}$ ergibt sich eine Gruppenlaufzeit von etwa 10-20 Zeitschritten, wobei diese für noch geringere Frequenzen stark ansteigt. Legt man das Ergebnis aus Kapitel 4 zugrunde, wonach die Laufzeit der schnellsten korrelierten Störungen zwischen dem Referenzsensor und dem Kompensationsaktuator etwa 40 Zeitschritte beträgt, sind hinsichtlich der Kausalität keine Probleme zu erwarten.

Bei jedem Versuch wurden die Zeitsignale am Referenz- und Fehlersensor aufgenommen (4 $10^{5}$ Abtastwerte, entsprechend etwa 3,2 min.). Desweiteren wurde nach Aufnahme der Zeitsignale die Schwankungsgeschwindigkeit $1 \mathrm{~cm}$ stromab des Fehlersensors in Abhängigkeit vom Wandabstand (Profile) gemessen. Diese Messungen wurden bei jeder Amplitudenstufe (0,1-0,2-0,4-0,8) für vier verschiedene Fälle durchgeführt. Zuerst wurden die Zeitsignale und Störprofile für den natürlichen Störhintergrund, also ohne (künstliche) Störungen, durchgeführt. Danach wurde die künstliche Störung eingeschaltet und die Messungen wiederholt (mit Störung). Die Messungen für den Fall mit Störkompensation wurden nach abgeschlossener Adaption des adaptiven Filters durchgeführt. Für die Aufnahme des Kompensationssignals wurde das während der Messung mit künstlicher Störung aufgenommene Referenzsignal mit dem, aus der Kompensationsmessung erhaltenen Filter gefiltert und bei ausgeschalteter Störanregung über den Kompensationsaktuator ausgegeben. Neben dem Vergleich der spektralen Leistungsdichten des Störsignals und des Kompensationssignals am Fehlersensor, konnte 


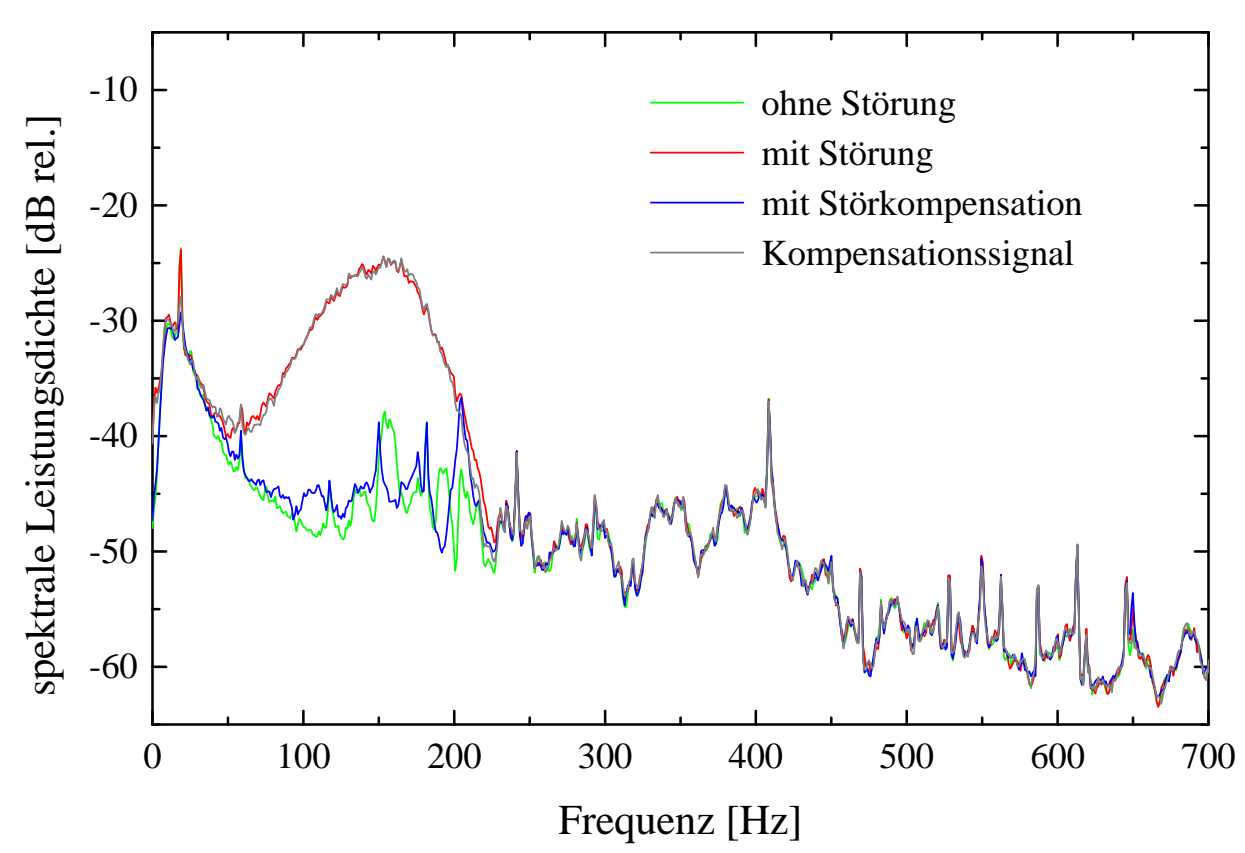

Abbildung 5.2: Spektrale Leistungsdichte des Fehlersensorsignals, Amplitude: 0,1

somit auch ihre Phasenbeziehung bestimmt werden.

In Abbildung 5.2 sind die Leistungsspektren des Fehlersignals für die kleinste Störamplitude $(0,1)$ dargestellt. Der Vergleich zwischen den Spektren ohne (grün) und mit künstlicher Störanregung (rot) zeigt eine deutliche spektrale Anhebung zwischen etwa $50 \mathrm{~Hz}$ und $220 \mathrm{~Hz}$ um bis zu $20 \mathrm{~dB}$. Ein Vergleich mit dem Instabilitätsdiagramm 4.4 zeigt, daß dies zumindest ungefähr der Bereich ist, in dem nach linearer Stabilitätstheorie eine Anfachung von TS-Wellen vorausgesagt wird. Schaltet man nun die dynamische Stabilisierung ein und läßt das Filter auskonvergieren, so ergibt sich eine sehr gute Kompensation der Störungen. Als Restfehler (blau) verbleibt im wesentlichen das Grundrauschen, das ohne künstliche Störanregung vorhanden ist. Wie nicht anders zu erwarten, zeigt das Kompensationssignal (grau) die gleiche spektrale Verteilung, wie die zu kompensierende Störung (rot). Da die Signale für die Berechnung der Leistungsspektren nicht absolut gemessen wurden ([dB rel.]), lassen sich relevante Aussagen nur durch den Vergleich der Spektren für unterschiedliche Störsituationen ableiten.

In Abbildung 5.3 sind die Ergebnisse der zugehörigen Profilmessung dargestellt. Es ist der RMS-Wert der Schwankungsgeschwindigkeit in x-Richtung $u_{\text {rms }}$ bezogen auf die Strömungsgeschwindigkeit am Grenzschichtrand $U_{e}$ über der wandnormalen Koordinate $y$ aufgetragen, wobei diese mit der Blasius-Länge $\delta_{r}$ dimensionslos gemacht wurde. Es zeigt sich die Ausbildung eines wandnahen Maximums bei etwa $\eta=y / \delta_{r}=1$ infolge der Störanregung (rot), das vollständig kompensiert 


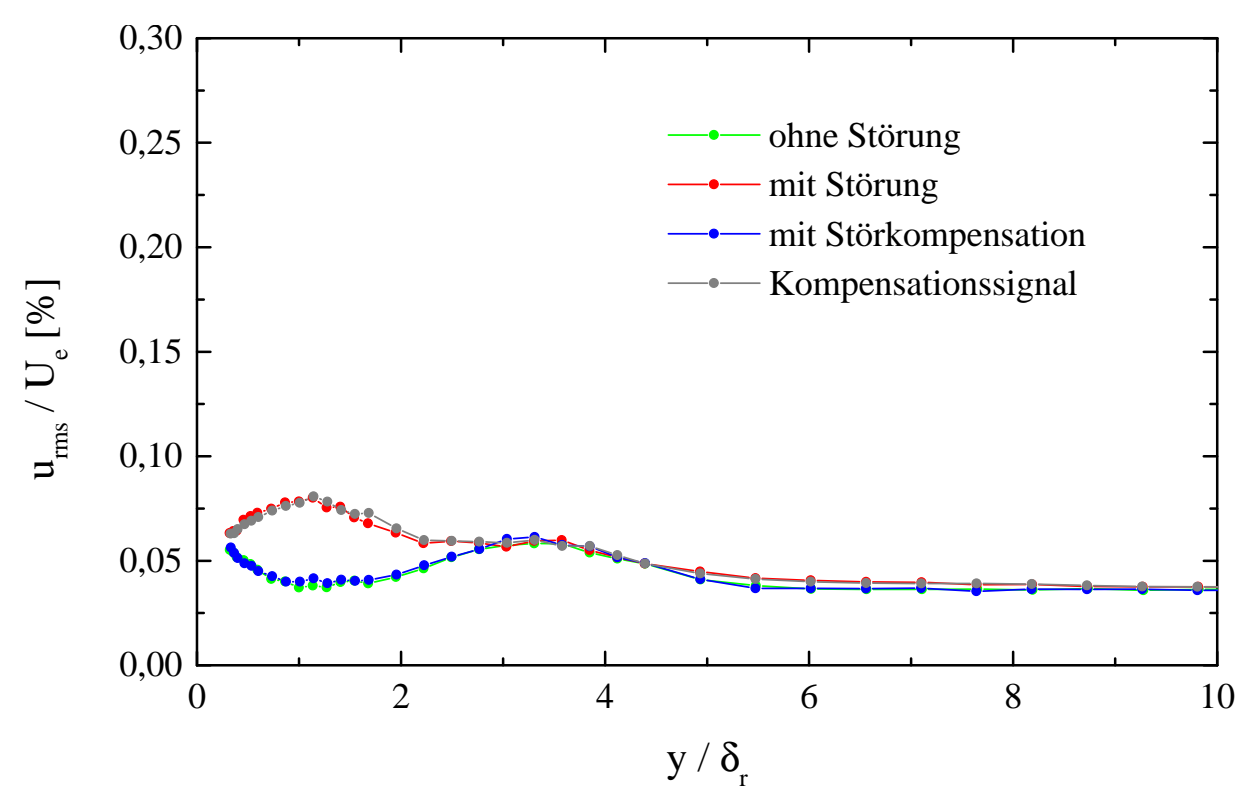

Abbildung 5.3: Störprofile, Amplitude: 0,1

werden kann (blau).

Man kann nun die erreichte Dämpfung, die sich als Differenz zwischen den Leistungsspektren mit und ohne Störung ergibt, mit der nach Gleichung 3.40 anhand der Kohärenz zwischen dem Referenz- und dem Fehlersignal vohergesagten Dämpfung vergleichen. Bei der praktischen Messung (Analysator) oder Berechnung der Kohärenz zeigt sich jedoch, daß ihr Wert insbesondere im Fall eines geringen Signal-Rausch-Abstandes am Referenzsensor stark von der Fensterlänge und der Gesamtlänge des aufgenommenen Signals abhängt. Daher wurde zur Bestimmung der theoretischen Dämpfung unter Zugrundelegung vollständig linearer Übertragungsfunktionen ein anderes Verfahren verwendet, das, obwohl es theoretisch äquivalent sein sollte, dennoch zuverlässigere Werte lieferte.

Hierfür wurde anhand der gemessenen Zeitsignale die Strecke zwischen Referenzund Fehlersensor nachgebildet (FIR-Filter, SFTF) und aus dem sich ergebenden Fehler die mögliche Dämpfung $D_{\mathrm{L}(\text { Linear),1 }}$ berechnet. Der Vergleich der experimentell erreichten (blau) und theoretisch vorhergesagten (schwarz) Dämpfung in Abbildung 5.4 zeigt bis etwa $200 \mathrm{~Hz}$ eine vollständige Übereinstimmung. Bei höheren Frequenzen jedoch wird für Störanteile, die auch ohne Störanregung vorhanden sind (vgl. Abb.5.2), eine Dämpfung vorhergesagt, die im Experiment nicht erreicht werden konnte.

Eine Ursache für diese Diskrepanz könnte sein, daß sich diese höherfrequenten Störanteile deutlich schneller ausbreiten oder sogar gleichzeitig an beiden Sensoren detektiert werden (z.B. Druckschwankungen), wodurch sie bei der Streckener- 


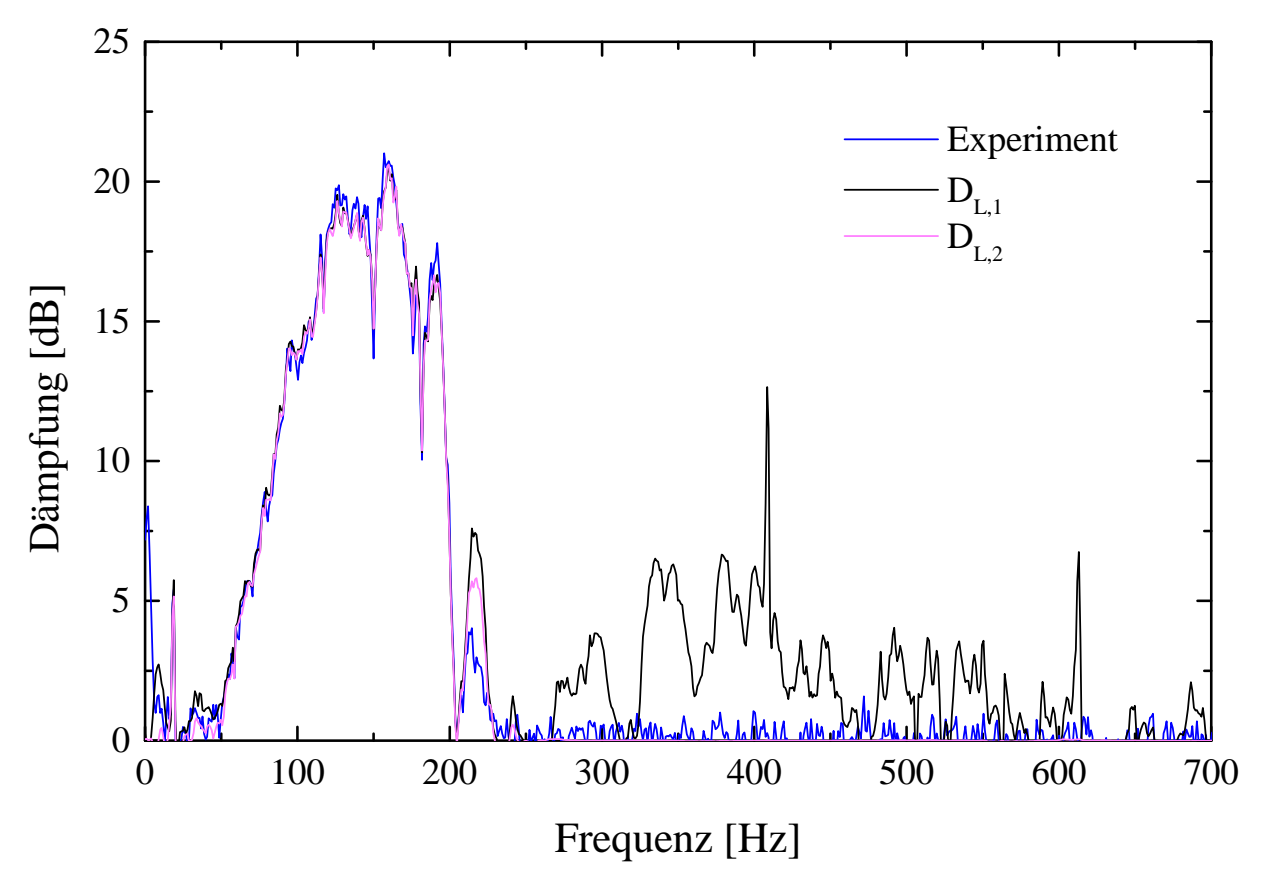

Abbildung 5.4: Vergleich der im Experiment erzielten Dämpfung mit der aus der Kohärenz berechneten möglichen Dämpfung, Amplitude: 0,1

kennung zwar korreliert werden können, eine Beeinflussung bei der dynamischen Stabilisierung aufgrund der Signallaufzeit im Beeinflussungszweig jedoch nicht möglich ist. Bei der theoretische Berechnung der Dämpfung kann diese Laufzeit durch die Filterung des Referenzsignales mit der im Versuch erkannten Sekundärstreckenschätzung (s. Abb. 5.5) berücksichtigt werden. Man erhält so ein zur dynamischen Stabilisierung vollständig äquivalentes Modell (vgl. Abb.3.5).

Die so erhaltene Vorhersage der möglichen Dämpfung $D_{\mathrm{L}, 2}$ ist in Abb. 5.4 (violett) eingetragen und zeigt im gesamten Frequenzbereich eine sehr gute Übereinstimmung mit dem experimentellen Ergebnis. Es ist noch anzumerken, daß die Filterung des Referenzsignals mit der Sekundärstreckenschätzung, deren Übertragungsfunktion in Abbildung 5.6 dargestellt ist, neben einer Verzögerung des Signals auch eine Dämpfung höherer Frequenzen bewirkt. Prinzipiell könnte auch diese Dämpfung der höherfrequenten Signalanteile im Referenzsignal bewirken, daß eine Nachbildung dieser Anteile am Fehlersensor nicht mehr möglich ist. Eine vergleichende Rechnung, bei der das Referenzsignal mit einer, der minimalen Gruppenlaufzeit der Sekundärstrecke entsprechenden, reinen Verzögerung gefiltert wurde, wodurch der Dämpfungseinfluß ausgeschlossen wird, lieferte jedoch das gleiche Ergebnis.

Verdoppelt man nun die Störamplitude $(0,2)$, so erhält man die in Abbildung 5.7 dargestellten Leistungsspektren am Fehlersensor. Mit Störung zeigt sich im 


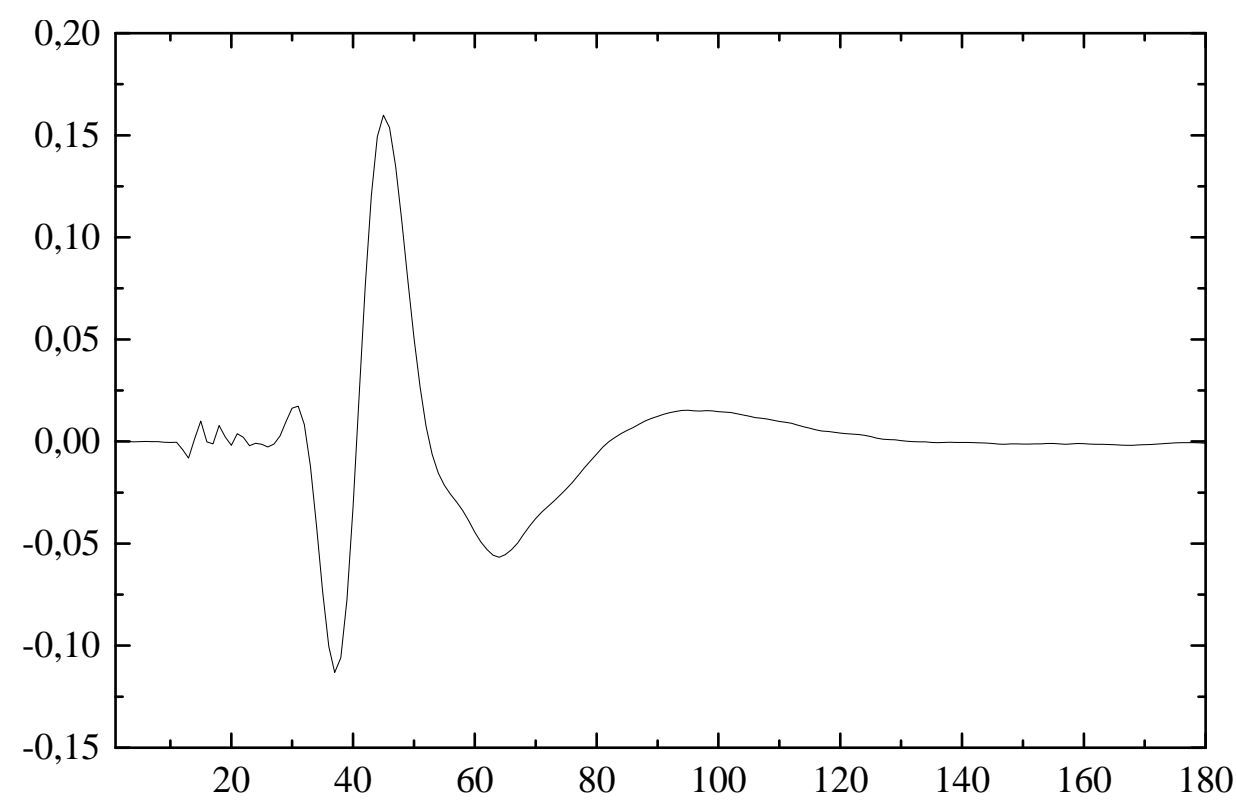

Filterkoeffizient Nr.

Abbildung 5.5: Impulsantwort der Sekundärstrecke

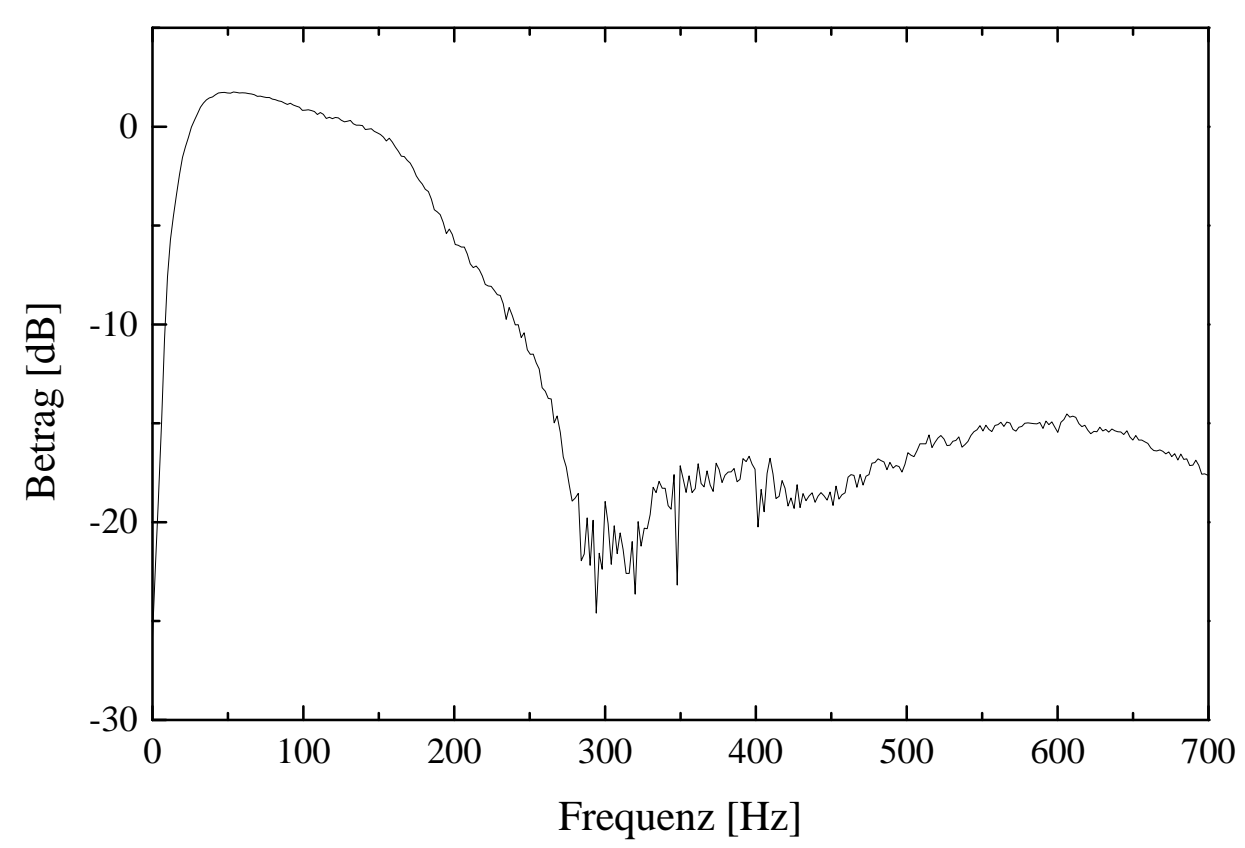

Abbildung 5.6: Frequenzgang der Sekundärstrecke 


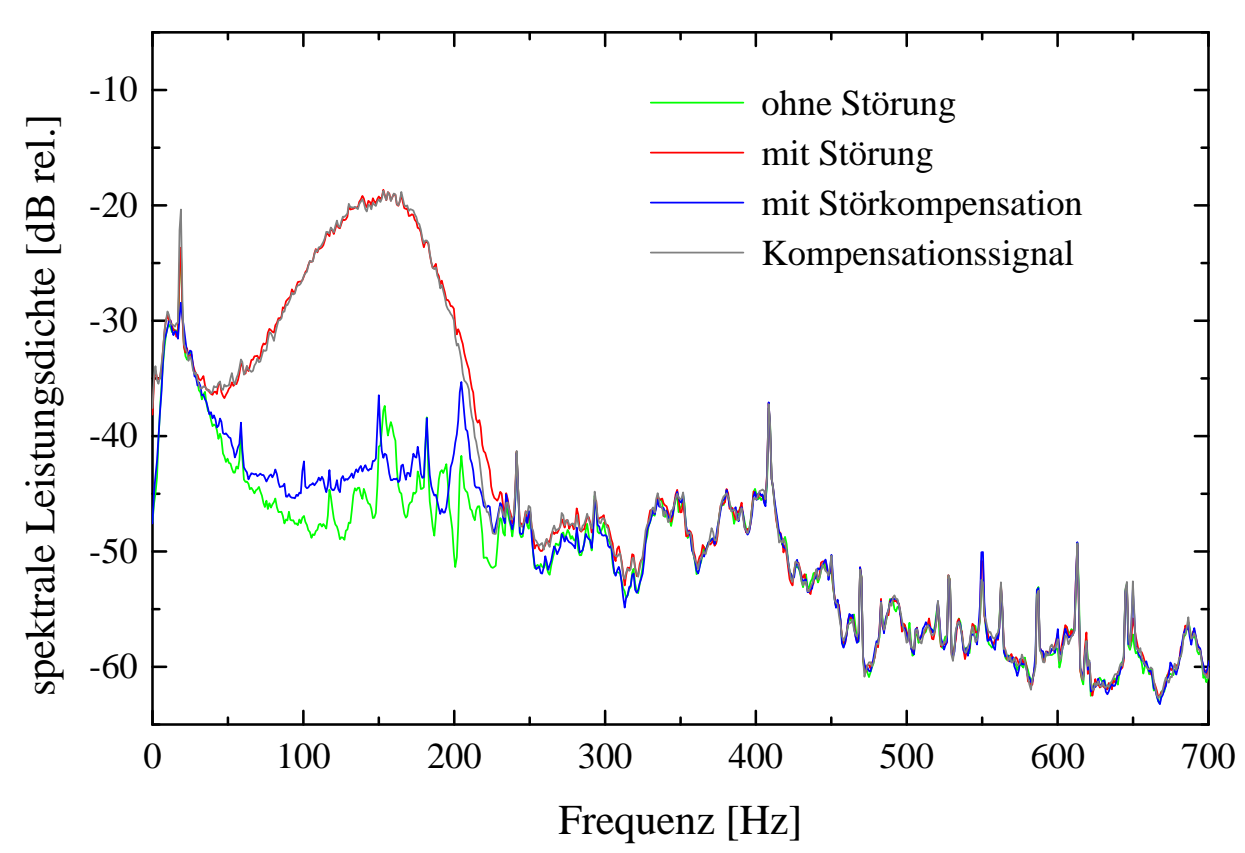

Abbildung 5.7: Spektrale Leistungsdichte des Fehlersensorsignals, Amplitude: 0,2

Maximum wie erwartet eine um etwa $6 \mathrm{~dB}$ höhere Anhebung als für die Amplitude 0,1. Zusätzlich läßt sich eine geringfügige Pegelerhöhung zwischen etwa $250 \mathrm{~Hz}$ und $300 \mathrm{~Hz}$ erkennen. Nach der Störkompensation verbleiben im Bereich um $100 \mathrm{~Hz}$ etwas größere Reststörungen als für die Amplitude 0,1.

Ähnliche Ergebnisse liefert die Profilmessung (Abb. 5.8). Neben einer Vergrößerung des maximalen RMS-Wertes um den Faktor zwei gegenüber der vorigen Messung auf 0,15\%, zeigt sich für diese Amplitude deutlich das charakteristische Nebenmaximum der TS-Mode. Die Ursache für das bei allen Messungen auftretende Maximum bei etwa $\eta=3,5$ ist nicht bekannt. Im Spektrum des Hitzdrahtsignals macht es sich durch, bei diesen Wandabständen deutlich hervortretende Störungen, für Frequenzen unterhalb von $100 \mathrm{~Hz}$ bemerkbar. Da es im Bereich des Phasensprungs der TS-Mode liegt, überdeckt es diesen insbesondere bei kleinen Amplituden.

Während sich für das Nebenmaximum durch die dynamische Stabilisierung eine vollständige Kompensation ergibt, verbleibt im Bereich des wandnahen Maximums eine kleine Reststörung, wobei das Kompensationssignal (grau) auch nicht die Stärke der eigentlichen Störung (rot) erreicht.

Interessant ist der Vergleich der erzielten Dämpfung (Spektralbereich) mit der Vorhersage nach linearer Theorie $D_{\mathrm{L}, 2}$, da sowohl im Frequenzbereich um $100 \mathrm{~Hz}$, als auch im Bereich der kleinen Pegelerhöhung bei $300 \mathrm{~Hz}$ eine größere Dämpfung erreicht wurde, als theoretisch vorhergesagt (s. Abb 5.9). 


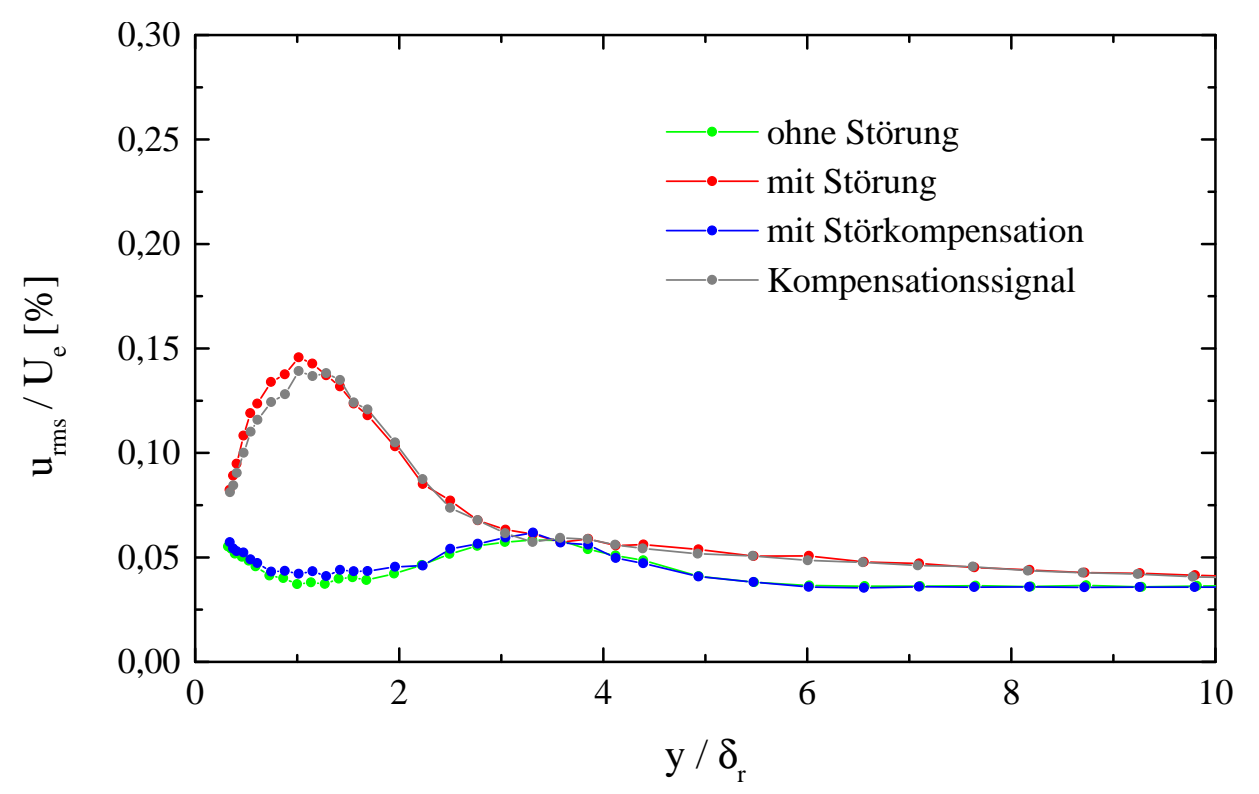

Abbildung 5.8: Störprofile, Amplitude: 0,2

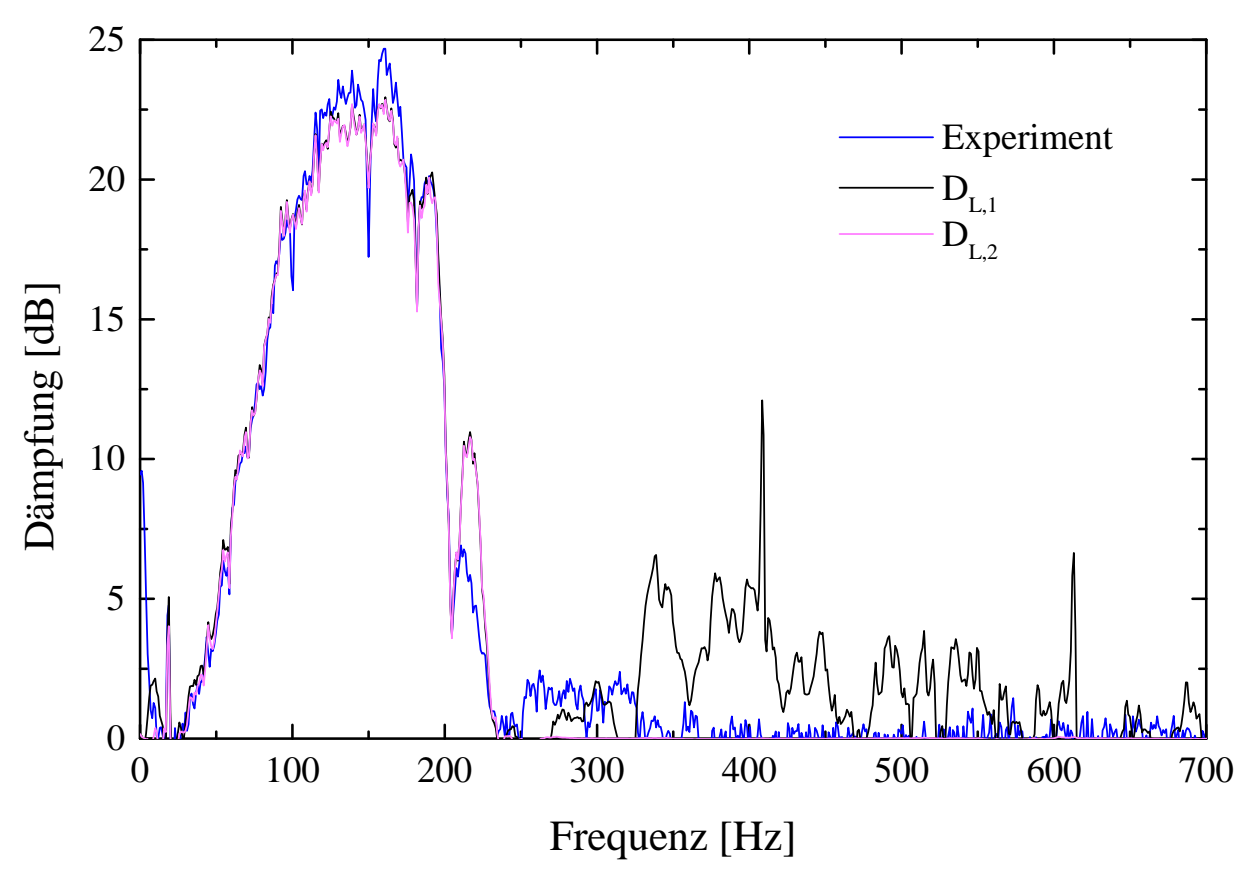

Abbildung 5.9: Vergleich der im Experiment erzielten Dämpfung mit der aus der Kohärenz berechneten möglichen Dämpfung, Amplitude: 0,2 


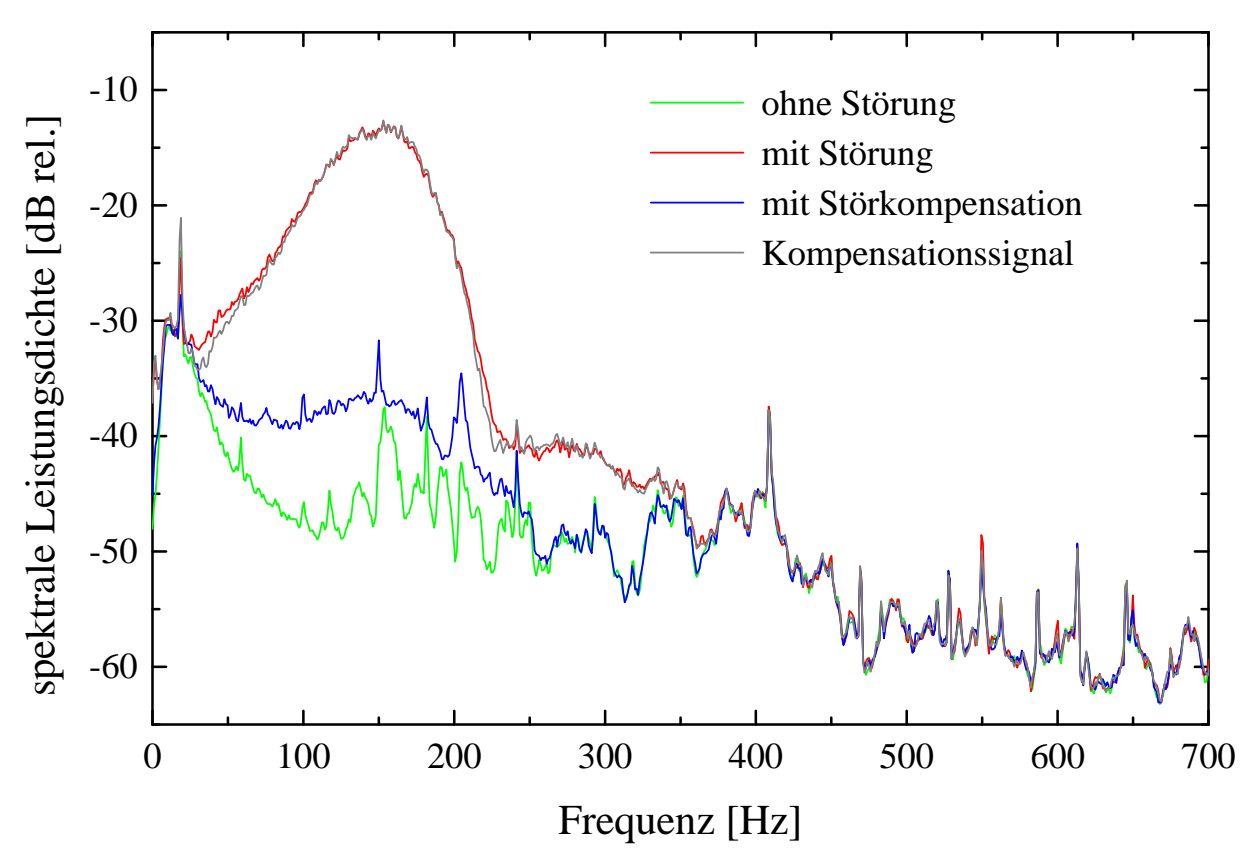

Abbildung 5.10: Spektrale Leistungsdichte des Fehlersensorsignals, Amplitude: 0,4

Weitaus deutlicher können die beschriebenen Effekte nach einer weiteren Verdopplung der Störamplitude auf 0,4 beobachtet werden. Im Spektrum (Abb. 5.10) zeigt sich im Fall der Störanregung sehr deutlich ein Störanteil um $300 \mathrm{~Hz}$, also mit der doppelten Frequenz des Maximums im TS-Grundfrequenzbereich (150 $\mathrm{Hz}$ ), der bis zu $10 \mathrm{~dB}$ über der Leistung im ungestörten Fall liegt.

Betrachtet man in Abb. 5.11 die geringe lineare Dämpfungsvorhersage für diesen Frequenzbereich, so ist die einfachste Erklärung, daß diese Störkomponenten aufgrund von nichtlinearen Prozessen bei der Ausbreitung und Anfachung der TS-Wellen aus diesen hervorgegangen sind (Summenfrequenzen). Schaltet man nun die dynamische Stabilisierung ein, werden diese Störungen vollständig „,kompensiert", während im Grundfrequenzbereich nichtkompensierbare Reststörungen verbleiben, wobei sich die Leistung dieser Störungen im Vergleich mit der Störanregung 0,2 deutlich erhöht hat. Die vollständige Dämpfung der höherharmonischen Komponenten läßt sich dadurch erklären, daß durch die Kompensation der linear korrelierten TS-Wellen, ihre Amplitude hinter dem Kompensationsaktuator so klein wird, daß hier Nichtlinearitäten keine Rolle mehr spielen. Eventuell schon vor dem Kompensationsaktuator entstandene höherharmonische Störungen befinden sich in einem Frequenzbereich, in dem sie zumindest über den TS-Instabilitätsmechanismus nicht angefacht werden, und können somit bis zum Fehlersensor abklingen. 


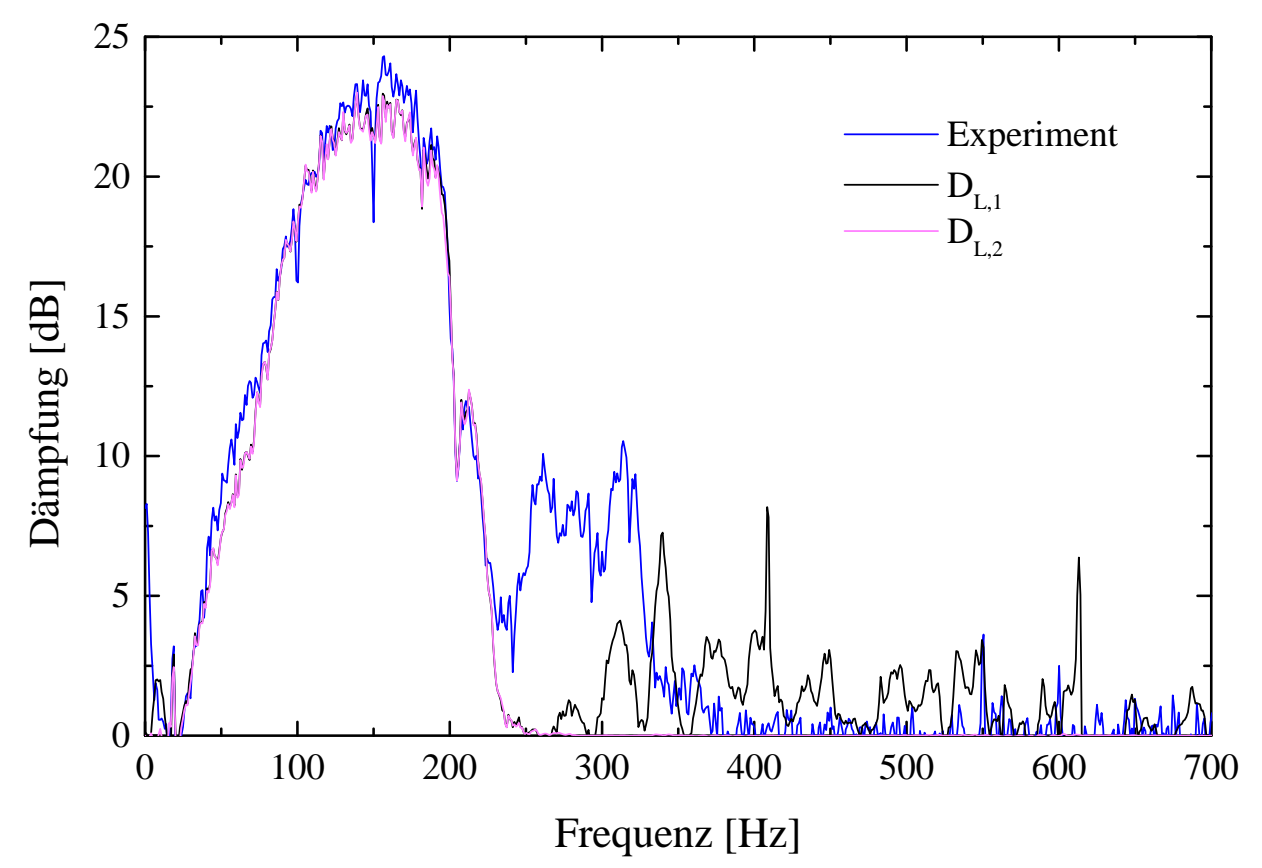

Abbildung 5.11: Vergleich der im Experiment erzielten Dämpfung mit der aus der Kohärenz berechneten möglichen Dämpfung, Amplitude: 0,4

Bei den Profilmessungen (Abb. 5.12) zeigt sich für das Nebenmaximum eine vollständige Kompensation, während im wandnahen Bereich nichtkompensierbare Störungen verbleiben.

Vergrößert man die Störamplitude auf 0,8 , so treten neben einer deutlichen Erhöhung sowohl der höherharmonischen Störung im künstlich gestörten Fall und insbesondere auch der im Grundfrequenzbereich verbleibenden, nichtkompensierbaren Reststörungen, bei tiefen Frequenzen weitere Störanteile hinzu (s. Abb. 5.13).

Ihre Lage im Frequenzbereich um etwa $70 \mathrm{~Hz}$ deutet darauf hin, daß es sich hierbei um Differenzfrequenzen oder Subharmonische handelt. Interessant ist, daß die Leistung des separat generierten Kompensationssignals (grau) in diesem Frequenzbereich deutlich geringer ist, als die Leistung im Fall der künstlichen Störanregung (rot). Solange diese tieffrequenten Störanteile nicht schon im Bereich des Referenzsensors entstehen, haben sie keinen Einfluß auf die Übertragungsfunktion des adaptiven Filters, da sie nur eine unkorrelierte Störung am Fehlersensor darstellen (s. Abschnitt 3.4). Würden diese Störanteile erst hinter dem Kompensationsaktuator generiert werden, müßte daher die Leistung des Kompensationssignals und des Störsignals sowohl am Kompensationsaktuator und, nach Durchlaufen der gleichen Ausbreitungsprozesse auch am Fehlersensor, weitgehend identisch sein. Diese Überlegung zeigt, daß diese Anteile zumindest teilweise vor 


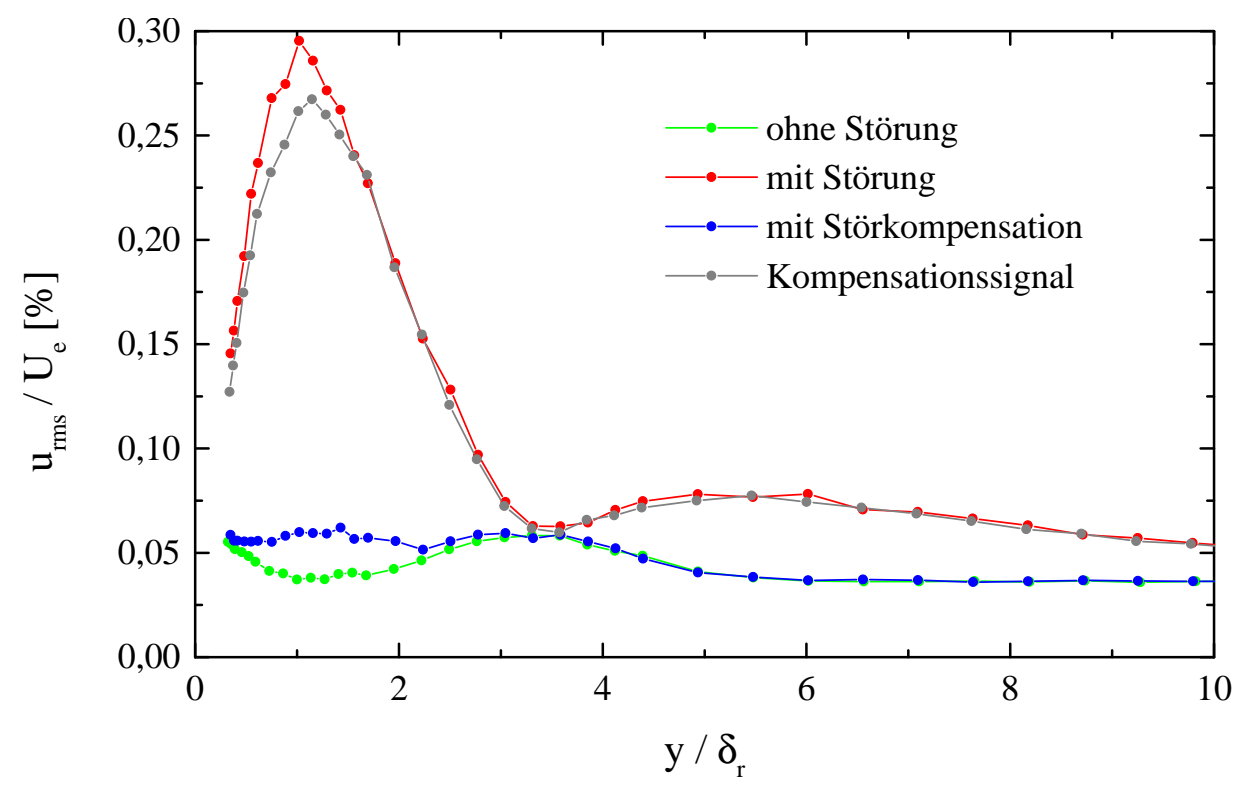

Abbildung 5.12: Störprofile, Amplitude: 0,4

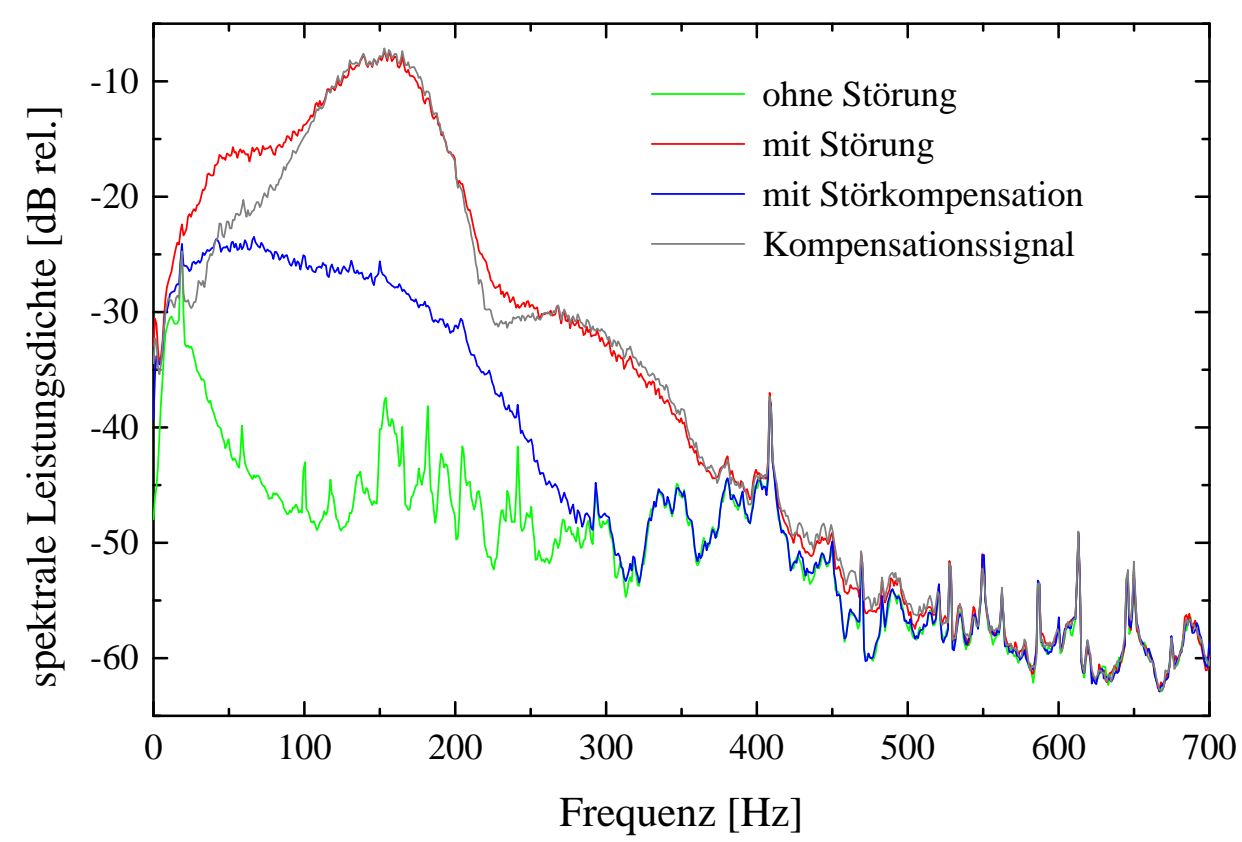

Abbildung 5.13: Spektrale Leistungsdichte des Fehlersensorsignals, Amplitude: 0,8 


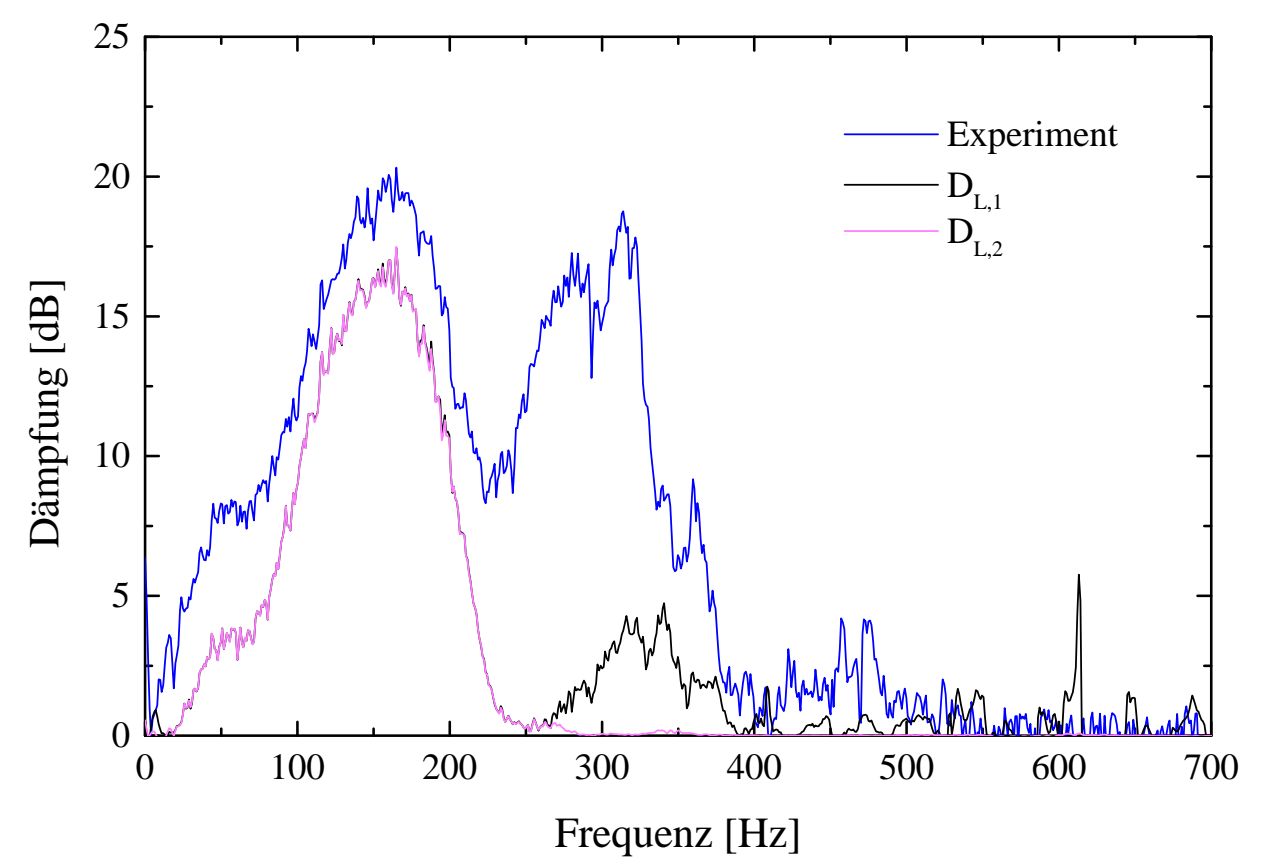

Abbildung 5.14: Vergleich der im Experiment erzielten Dämpfung mit der aus der Kohärenz berechneten möglichen Dämpfung, Amplitude: 0,8

dem Kompensationsaktuator entstanden sein müssen. Auch die große Differenz zwischen der unter Zugrundelegung linearer Prozesse erwarteten und tatsächlich erzielten Dämpfung in Abbildung 5.14 zeigt die deutliche Zunahme nichtlinear generierter Störanteile. Anhand der im Experiment erreichten Dämpfung läßt sich gut erkennen, daß im Bereich um $450 \mathrm{~Hz}$ auch drittharmonische Störungen existierten.

Die Profilmessungen (Abb. 5.15) zeigen, daß im wandnahen Maximum durch die Störkompensation eine Reduktion des RMS-Wertes von 0,85\% auf etwa 0,25\% erreicht wird, während sich für das Nebenmaximum eine vollständige Kompensation ergibt. Vergleicht man das Profil der Reststörung mit dem Profil (mit Störung) aus Abb. 5.12, das im wandnahen Maximum eine ähnliche Amplitude hat, so sieht man deutlich, daß sich die Profilform hinsichtlich des fehlenden Nebenmaximums von der Profilform einer TS-Welle unterscheidet.

In Abbildung 5.16 sind die Filterkoeffizienten bzw. Impulsantworten des adaptiven Filters dargestellt, die sich für die verschiedenen Amplituden ergeben haben. Bei einer gemessenen Gruppenlaufzeit von 40 Zeitschritten für die schnellsten linear korrelierten Störanteile und einer Gruppenlaufzeit von 10-20 Zeitschritten im Beeinflussungszweig ist zu erwarten, daß diese Impulsantwort eine Gruppenlaufzeit von etwa 20-30 Zeitschritten nachbildet. Tatsächlich liegen die betragsmäßig größten Koeffizienten wie erwartet zwischen Nr. 20 und Nr. 30. Dennoch zeigen 


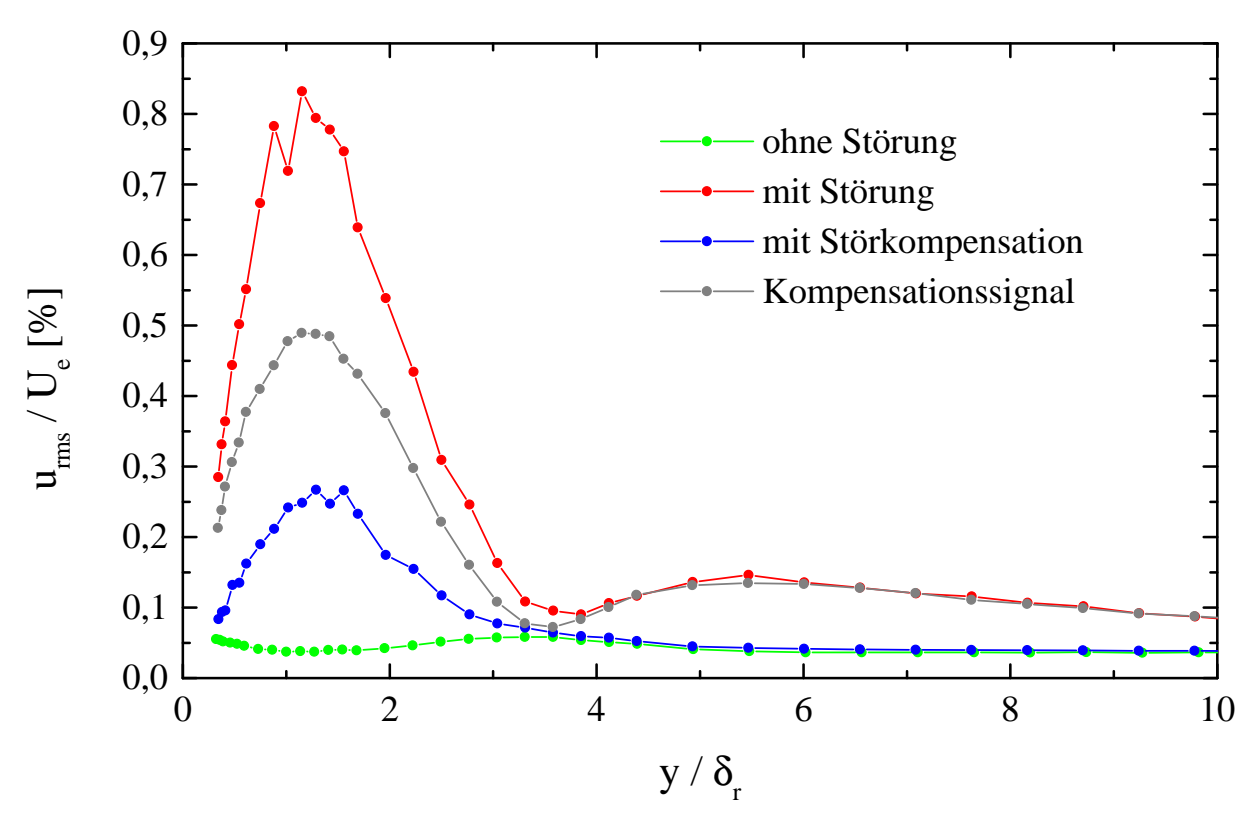

Abbildung 5.15: Störprofile, Amplitude: 0,8

sich auch für kürzere Laufzeiten $<10 \cdot \Delta t$ korrelierte Anteile, die sich anscheinend bis in den negativen Zeitbereich erstrecken, und von dem Filter nicht nachgebildet werden können. Es läßt sich jedoch zeigen, daß diese „akausalen“ Anteile aus der Invertierung des Beeinflussungszweiges bei Frequenzen mit einer großen Gruppenlaufzeit entstehen, die für die dynamische Stabilisierung bei den hier betrachteten Reynoldszahlen unwichtig waren.

Vergleicht man die Impulsantworten, so zeigt sich eine, wenn auch geringe, Amplitudenabhängigkeit des linearen Modells. Ein Vergleich des Betrages der Übertragungsfunktionen für die unterschiedlichen Amplituden (Abb. 5.17) zeigt im Maximum jedoch eine so geringe Variation (1 dB), daß eine Diskussion der einzelnen Prozesse, die zu dieser Änderung führen hier sicher nicht sinnvoll ist. Interessanter scheint die Änderung von bis zu $5 \mathrm{~dB}$ im tieffrequenten Bereich um etwa $50 \mathrm{~Hz}$ zwischen den Störamplituden 0,4 und 0,8 zu sein. Die hier sichtbare Vergrößerung des Betrages der Übertragungsfunktion deutet darauf hin, daß für diese Frequenzen auch schon vor dem Referenzsensor nichtlineare Prozesse eine Rolle spielen, wodurch ein Teil der auf diese Weise generierten Störungen zwischen den beiden Sensoren wieder linear korreliert ist. Die starken Veränderungen für Frequenzen oberhalb von $200 \mathrm{~Hz}$ sind Artefakte der Fouriertransformation, da vor der Transformation der Impulsantwort keine Fensterung vorgenommen wurde (spectral leakage).

Baumann [2] gibt als Phasendifferenz zwischen dem Störsignal und dem Kompensationssignal am Fehlersensor einen Wert von $124^{\circ}$ entsprechend 0, $68 \pi$ an, wobei 


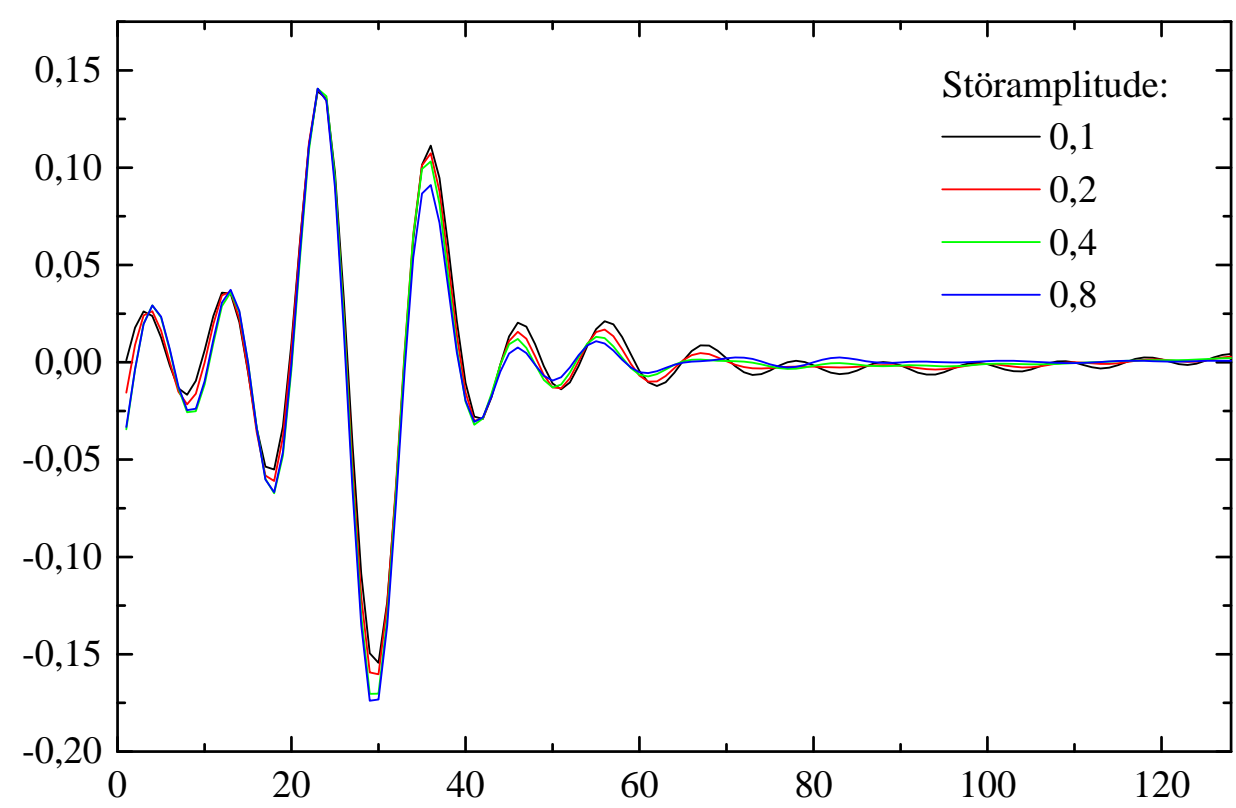

Filterkoeffizient Nr.

Abbildung 5.16: Impulsantwort des Hauptfilters

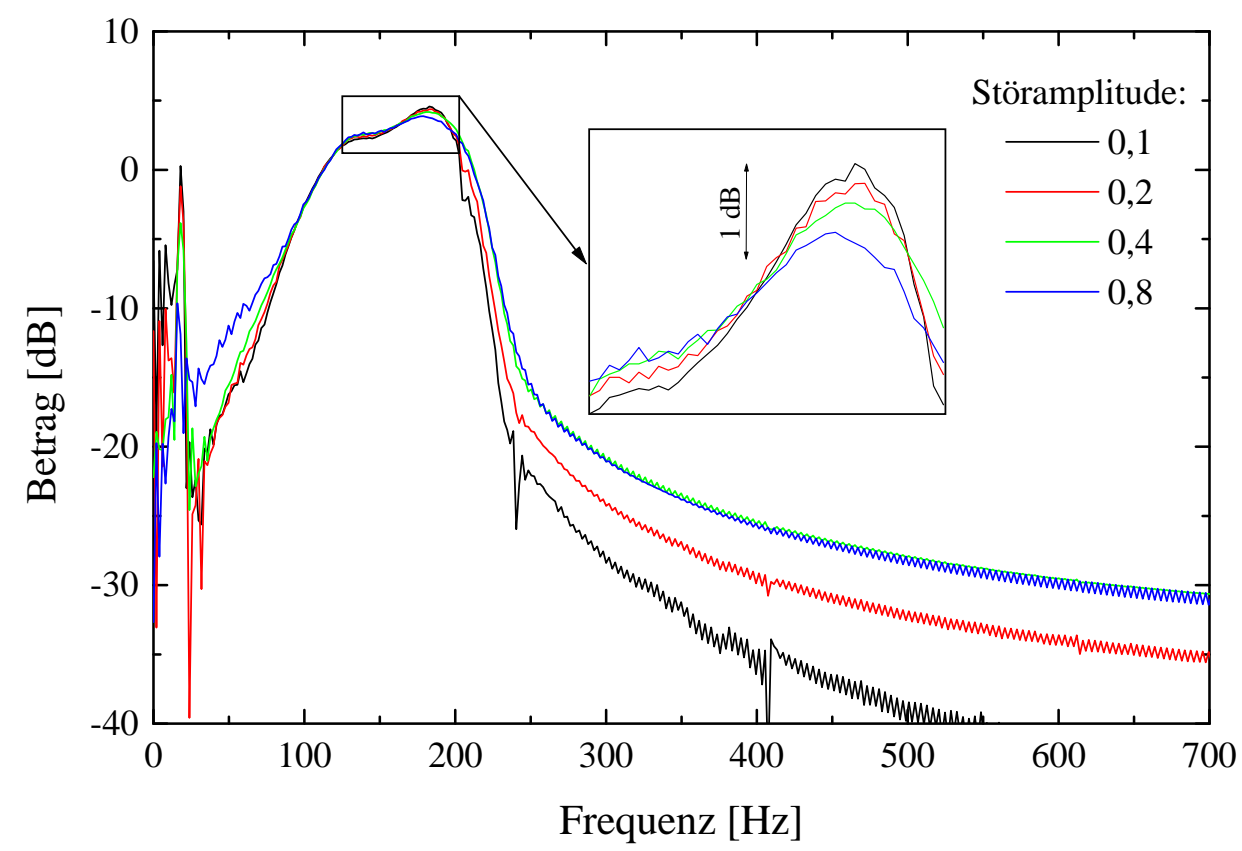

Abbildung 5.17: Frequenzgang des Hauptfilters 


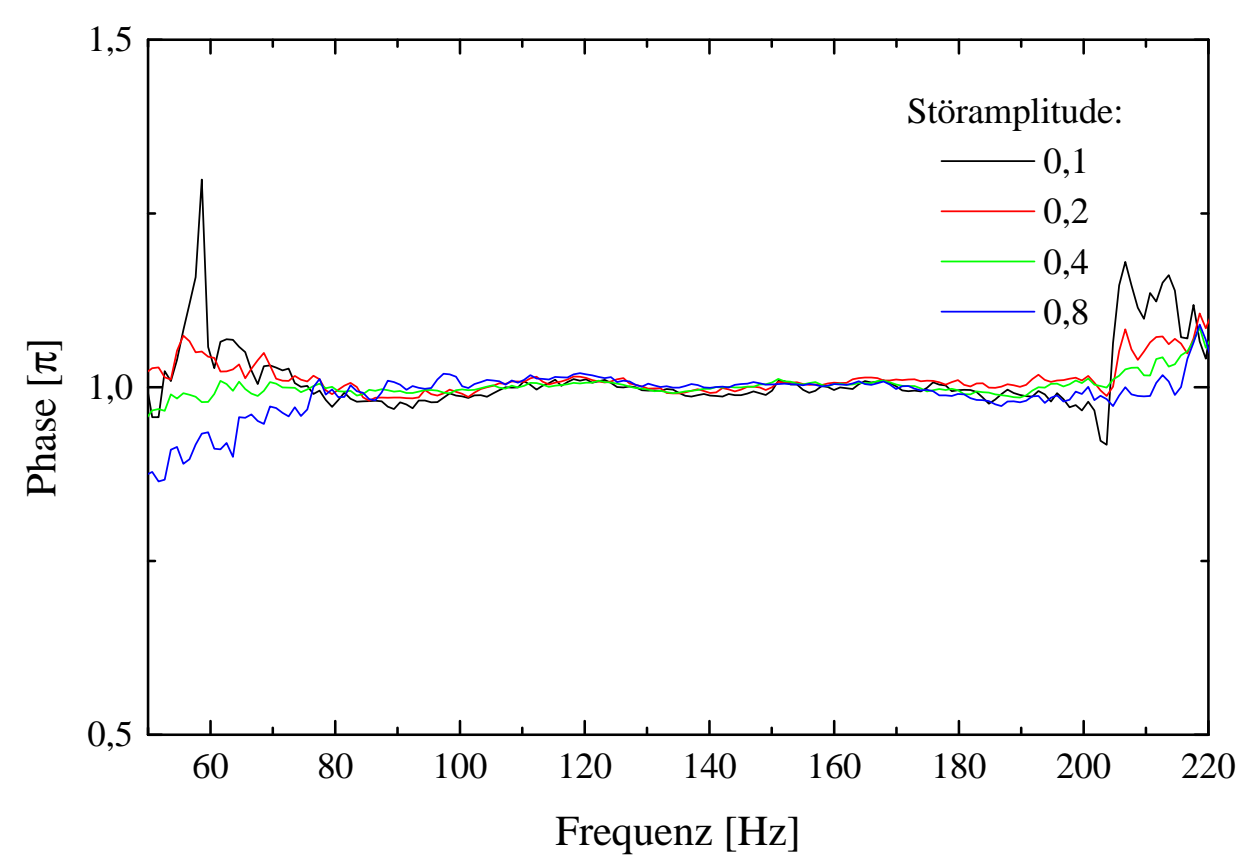

Abbildung 5.18: Phasendifferenz zwischen dem Stör- und dem Kompensationssignal

die Abweichung vom theoretisch erwarteten Wert $\pi$ durch nichtlineare Prozesse in unmittelbarer Aktuatornähe erklärt wird. Da dieses Ergebnis alle bisherigen Annahmen zur Wirkungsweise der dynamischen Stabilisierung in Frage stellt, wurde anhand der spektralen Kreuzleistungsdichte zwischen den gemessenen Stör- und Kompensationssignalen ihre Phasendifferenz bestimmt. Abbildung 5.18 zeigt, daß die Ergebnisse der hier durchgeführten Messungen im Grundfrequenzbereich für alle Störamplituden weitgehend der theoretisch erwarteten Phasendifferenz von $\pi$ entsprechen.

Es ist noch anzumerken, daß in der Literatur das Aufteten von Nichtlinearitäten häufig erst für Effektivwerte der Schwankungsgeschwindigkeit von etwa einem Prozent, bezogen auf die Außengeschwindigkeit, beschrieben wird. Hierbei werden allerdings sinusförmige Störsignale zugrundegelegt (Amplitude $=1.41 \cdot u_{\mathrm{rms}}$ ). Bei den verwendeten breitbandigen Störsignalen hängt die Beziehung zwischen der maximalen Störamplitude und dem Effektivwert stark von der vorliegenden Amplitudenverteilung ab, die hier nach der Ausbreitung der Störungen stromab deutlich von der Gleichverteilung des Anregungssignals abweicht. Für die höchste Störamplitude $(0,8)$ treten im wandnahen Maximum Amplituden von bis $\mathrm{zu} \pm 3 \%$ auf. 


\subsubsection{Nichtlinearität des Meßverfahrens}

Wie in Abschnitt 2.5 dargestellt wurde, besteht zwischen der Schwankungsgeschwindigkeit und der Anemometerausgangsspannung bei der Hitzdrahtmessung eine nichtlineare Beziehung. Dies gilt auch für die Messung von Wandschubspannungen mit einem Wandsensor [7]. Da dieser Zusammenhang für die mit dem Referenz- und Fehlersensor aufgenommenen Signale nicht linearisiert wurde, stellt sich die Frage inwieweit diese Nichtlinearität zu den beobachteten nichtkompensierbaren Reststörungen bei hohen Störamplituden führen kann. Die Nichtlinearität am Fehlersensor führt insbesondere für den Fall ohne Kompensation im Fehlersignal zur Generierung von Signalanteilen, die nicht linear mit dem Referenzsignal korreliert sind. Wie in Abschnitt 3.4 gezeigt wurde, hat eine solche unkorrelierte Störkomponente $v[n]$ allerdings keinen Einfluß auf das optimale Filter $W^{O}(z)$, das sich bei der Adaption einstellt. Außerdem wird die Störamplitude am Fehlersensor und damit der Einfluß dieser Nichtlinearität während der Adaption des Filters zunehmend geringer. Die Nichtlinearität des Fehlersensors kann daher als Ursache für das Auftreten von nichtkompensierten Störanteilen ausgeschlossen werden. Unkorrelierte Störanteile im Referenzsignal jedoch, die durch die Nichtlinearität des Referenzsensors entstehen können, führen im Vergleich mit der ungestörten Lösung zu einer Skalierung des Betrages der adaptierten Übertragungsfunktion. Somit kann diese Nichtlinearität prinzipiell eine verschlechterte Nachbildung der Übertragungsfunktion bewirken, wodurch die vollständige Kompensation auch linear korrelierter Grenzschichtstörungen nicht mehr gewährleistet wäre. In Experimenten zur dynamischen Stabilisierung, bei denen als Referenzsignal direkt das im Computer generierte Anregungssignal für den Störaktuator verwendet wurde, zeigten sich allerdings die gleichen nichtkompensierbaren Reststörungen. Daher sind die bei der dynamischen Stabilisierung für höhere Störamplituden beobachteten Phänomene nicht auf die Nichtlinearität der Signalaufnahme zurückzuführen.

\subsection{Einfluß nichtlinearer Störausbreitungs- prozesse}

Die Ergebnisse der Experimente zur dynamischen Stabilisierung zeigen, daß für größere Amplituden auch nichtlineare Ausbreitungsprozesse in der Grenzschicht eine Rolle spielen. Um genauere Aussagen zum Verhältnis von linear korrelierten zu nichtlinear generierten Störanteilen bei der Stromabausbreitung der TSInstabilitätswellen machen zu können, wurde ein Hitzdrahtsensor ausgehend von der Position direkt über dem Störaktuator schrittweise stromab verfahren $(\Delta x=1$ $\mathrm{cm}$ ) und dabei jeweils das mit dem Sensor gemessene Signal für die Störamplituden 0,2, 0,4 und 0,8 aufgenommen. Anhand der gemessenen Gleichspannung 
wurde der Wandabstand jeweils so eingestellt $\left(\bar{U} / U_{e}=\right.$ konst. $\left.\approx 0,3\right)$, daß sich der Sensor im Maximum der TS-Störwelle befand.

In Abbildung 5.19 sind die spektralen Leistungsdichten des Hitzdrahtsensorsignals für die Störamplituden 0,2 (oben) und 0,8 (unten) in Abhängigkeit vom Abstand zwischen dem Störaktuator und Hitzdrahtsensor aufgetragen. Der starke auch außerhalb des Aktuatornahfeldes auftretende Störanteil bei etwa $40 \mathrm{~Hz}$ resultiert aus einer Sondenschwingung, die durch eine Veränderung der Sondenhalterung am Stativ, wobei als Dämpfungselemente Moosgummistreifen verwendet wurden, zwar verringert, aber nicht vollständig bedämpft werden konnte.

Für beide Amplituden zeigt sich im gesamten Frequenzbereich bis zu einem Abstand von $2 \mathrm{~cm}$ ein starker Abfall der spektralen Leistung, bezogen auf die Meßposition direkt über dem Aktuator. Außerhalb dieses Nahfeldes führt der TSInstabilitätsmechanismus zur Anfachung eines relativ schmalen Frequenzbandes, dessen Maximum sich mit zunehmender Lauflänge zu tieferen Frequenzen verschiebt. Ein Vergleich der Spektren für die Amplitude 0,8 mit den Ergebnissen der Störamplitude 0,2, bei der die Ausbreitungsprozesse nach den Experimenten zur dynamischen Stabilisierung noch als weitgehend linear angenommen werden dürfen, zeigt insbesondere für tiefe Frequenzen $(50 \mathrm{~Hz}-100 \mathrm{~Hz})$ deutliche Unterschiede. Im Gegensatz zur Störamplitude 0,2, für die diese Spektralanteile bei der Ausbreitung stromab eine Dämpfung erfahren, läßt sich hier eine Verstärkung beobachten, die dazu führt, daß in diesem Frequenzbereich für die letzte Meßposition $(27 \mathrm{~cm})$ die stärksten Störanteile lokalisiert sind.

Um das Verhältnis von nichtlinear generierten Störanteilen zu den linear korrelierten Störungen für die Störamplituden 0,4 und 0,8 abzuschätzen, wurden die Meßergebnisse für die Frequenzen $75 \mathrm{~Hz}$ (Differenzfrequenzen, Subharmonische), $150 \mathrm{~Hz}$ (Grundfrequenzbereich) und $300 \mathrm{~Hz}$ (Summenfrequenzen, zweite Harmonische) genauer untersucht.

In Abbildung 5.20 ist die spektrale Leistungsdichte des Hitzdrahtsignals ohne (grün) und mit Störanregung (rot) für die Amplituden 0,4 (oben) und 0,8 (unten) bei der Frequenz $150 \mathrm{~Hz}(\Delta f \approx 2 \mathrm{~Hz})$ für die unterschiedlichen Positionen stromab aufgetragen. Für beide Amplituden zeigt sich ein von der Sensorposition weitgehend unabhängiges Grundstörniveau (grün). Betrachtet man den Fall mit Störung (rot), so sieht man nach dem deutlichen Abfall im Bereich des Nahfeldes eine Anfachung der Störung stromab, wobei nach dem Maximum zwischen dem Kompensationsaktuator und dem Fehlersensor ein leichter Abfall der spektralen Leistung bei dieser Frequenz zu beobachten ist. Der Fehler einer linearen Streckenerkennung zwischen dem Störanregungs- und dem Hitzdrahtsignal (blau) liegt für die ersten Sensorpositionen bei beiden Störamplituden etwa 15 dB unter dem Störsignal. Dies bedeutet, daß etwa $98 \%$ der Störleistung linear mit dem Anregungssignal korreliert sind. Für die Amplitude 0,4 bleibt dieses Verhältnis bei der Stromab-Traversierung in etwa erhalten, was bedeutet, daß die linearen und 

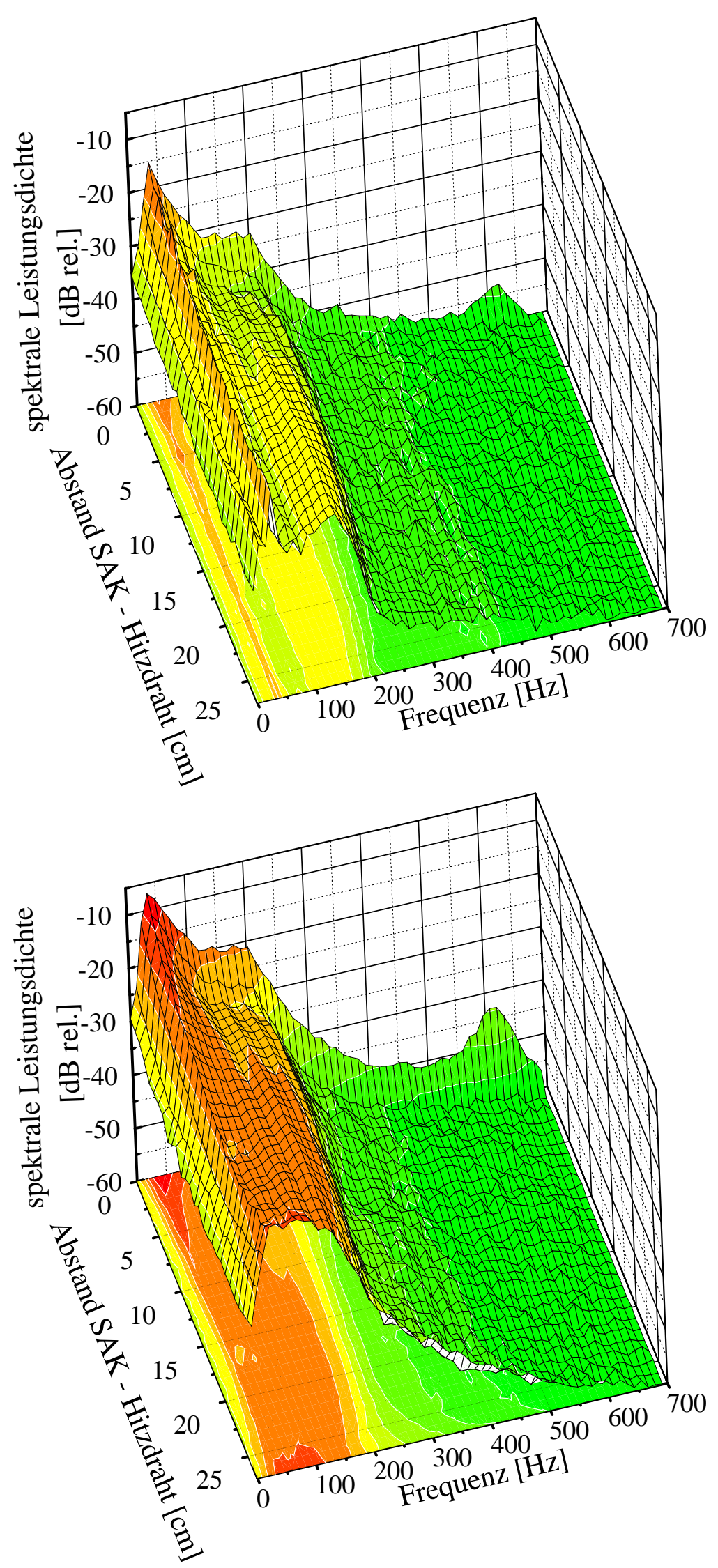

Abbildung 5.19: Spektrale Leistungsdichte des Hitzdrahtsignals für die Amplituden 0,2 (oben) und 0,8 (unten); Traversierung stromab 
nichtlinearen Störanteile in gleichem Maße ansteigen. Bei der doppelten Amplitude zeigt sich hingegen ein zunehmend stärkerer Einfluß der nichtlinear erzeugten Störungen, wobei für die letzte Meßposition nur noch etwa 70 \% der Störleistung mit einem linearen Modell nachgebildet werden können.

In Abbildung 5.21 sind die eben erläuterten Ergebnisse für die Frequenz 300 $\mathrm{Hz}$ aufgetragen. Für die Amplitude 0,4 sind in diesem Frequenzbereich im wesentlichen direkt vom Störaktuator erzeugte Störkomponenten zu erkennen, die durch das lineare Modell vollständig nachgebildet werden können und bis zum Referenzsensor (RS) abklingen. Auch für die Störamplitude 0,8 fällt die Leistung zunächst ab, wächst dann hinter dem Referenzsensor aber wieder stark an, wobei der Fehler der linearen Erkennung zeigt, daß diese Signalkomponenten für höhere Reynoldszahlen keinen linearen Zusammenhang mit der Störanregung mehr aufweisen. Die Experimente zur dynamischen Stabilisierung haben jedoch gezeigt, daß diese Störanteile über die Kompensation der erzeugenden Störungen im Grundfrequenzbereich mitgedämpft werden, da sie sich nicht im angefachten Frequenzbereich befinden.

Die Differenzfrequenzbildung hingegen erzeugt Störungen, die auch nach der linearen Theorie angefacht werden, da sich der Anfachungsbereich mit zunehmender Lauflänge zu tieferen Frequenzen verschiebt und sie sich daher auch unabhängig von den erzeugenden Störungen ausbreiten können. Für die Störamplitude 0,4 zeigt sich in Abbildung 5.22, daß die Leistung der bei $75 \mathrm{~Hz}$ erzeugten Signalanteile bis zum Kompensationsaktuator abfällt und vollständig linear mit dem Anregungssignal korreliert ist. Hinter dem Kompensationsaktuator steigt der Restfehler leicht an und zeigt damit nichtlineare Ausbreitungsprozesse an, die bei diesen Frequenzen Störungen generieren. Für die doppelte Störamplitude spielen Nichtlinearitäten schon im Bereich des Referenzsensors eine Rolle und bestimmen mit zunehmender Reynoldszahl maßgeblich die Störerzeugung in diesem Frequenzbereich. Hinter dem Kompensationsaktuator steigt die Störleistung stark an, wobei an der letzten Meßposition $3 \mathrm{~cm}$ hinter dem Fehlersensor nur noch etwa $30 \%$ der Gesamtleistung linear aus dem Anregungssignal abzuleiten sind. 

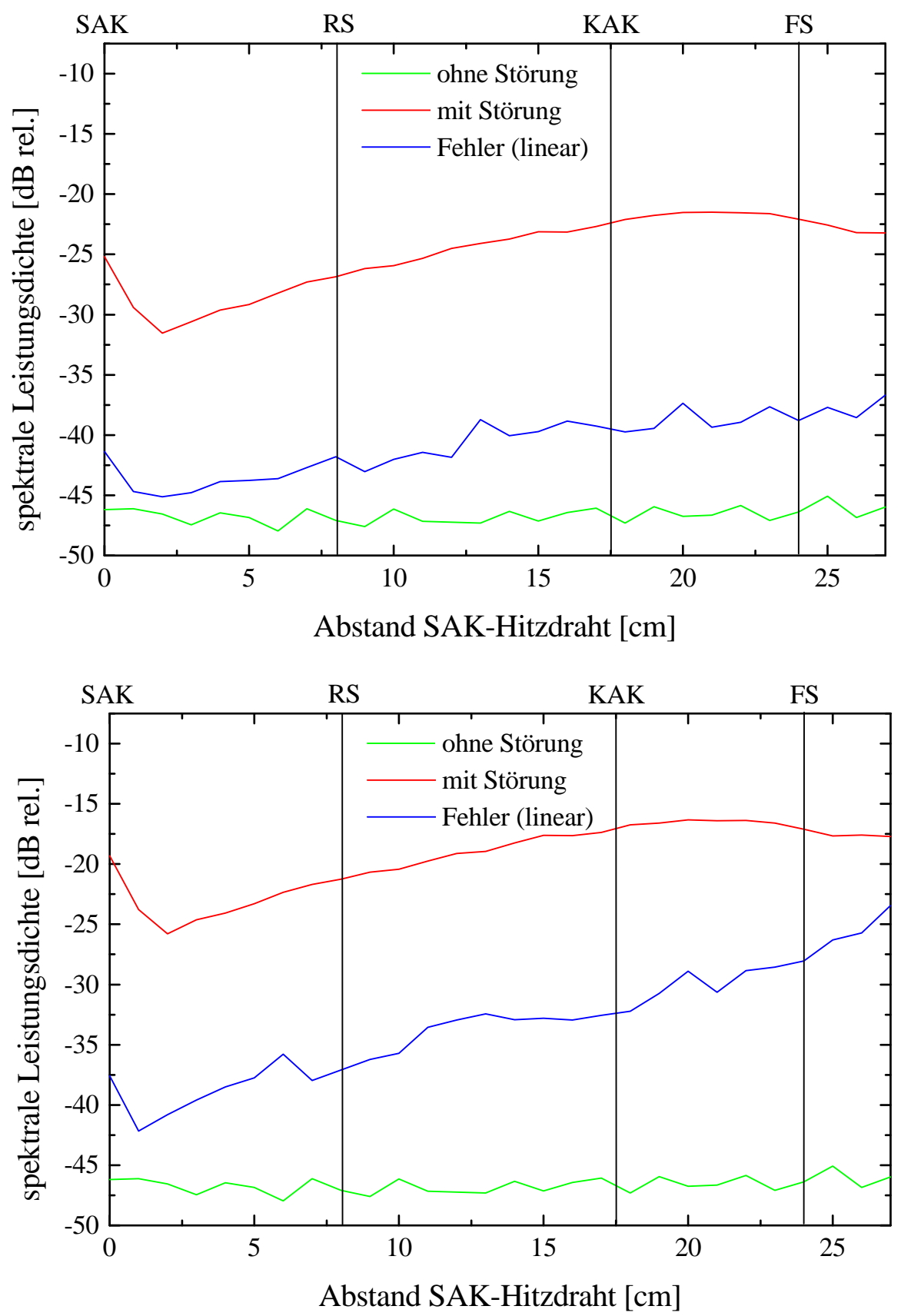

Abbildung 5.20: Spektrale Leistungsdichte des Hitzdrahtsignals (150 Hz) Amplitude: 0,4 (oben) und 0,8 (unten); Traversierung stromab 

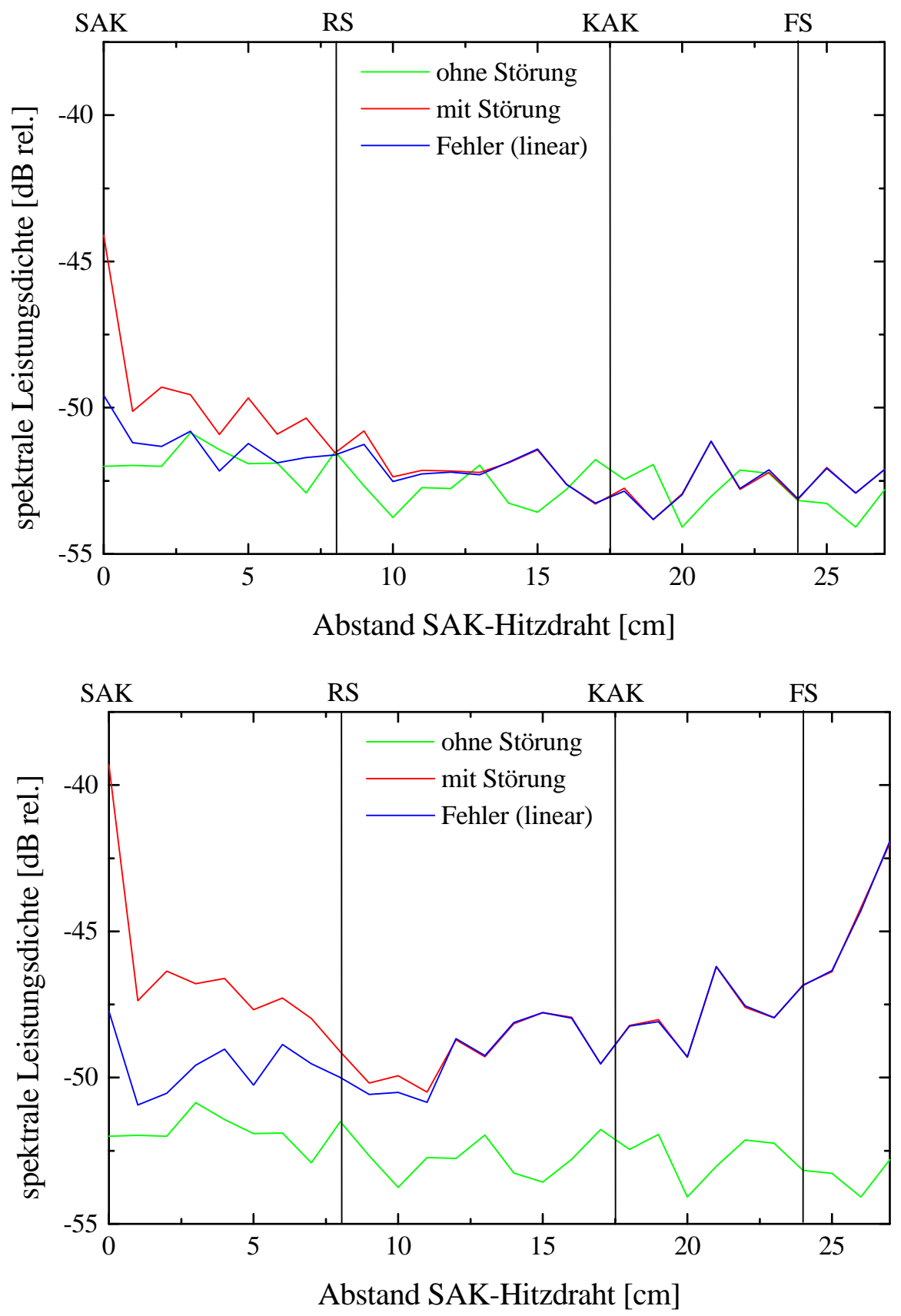

Abbildung 5.21: Spektrale Leistungsdichte des Hitzdrahtsignals (300 Hz) Amplitude: 0,4 (oben) und 0,8 (unten); Traversierung stromab 

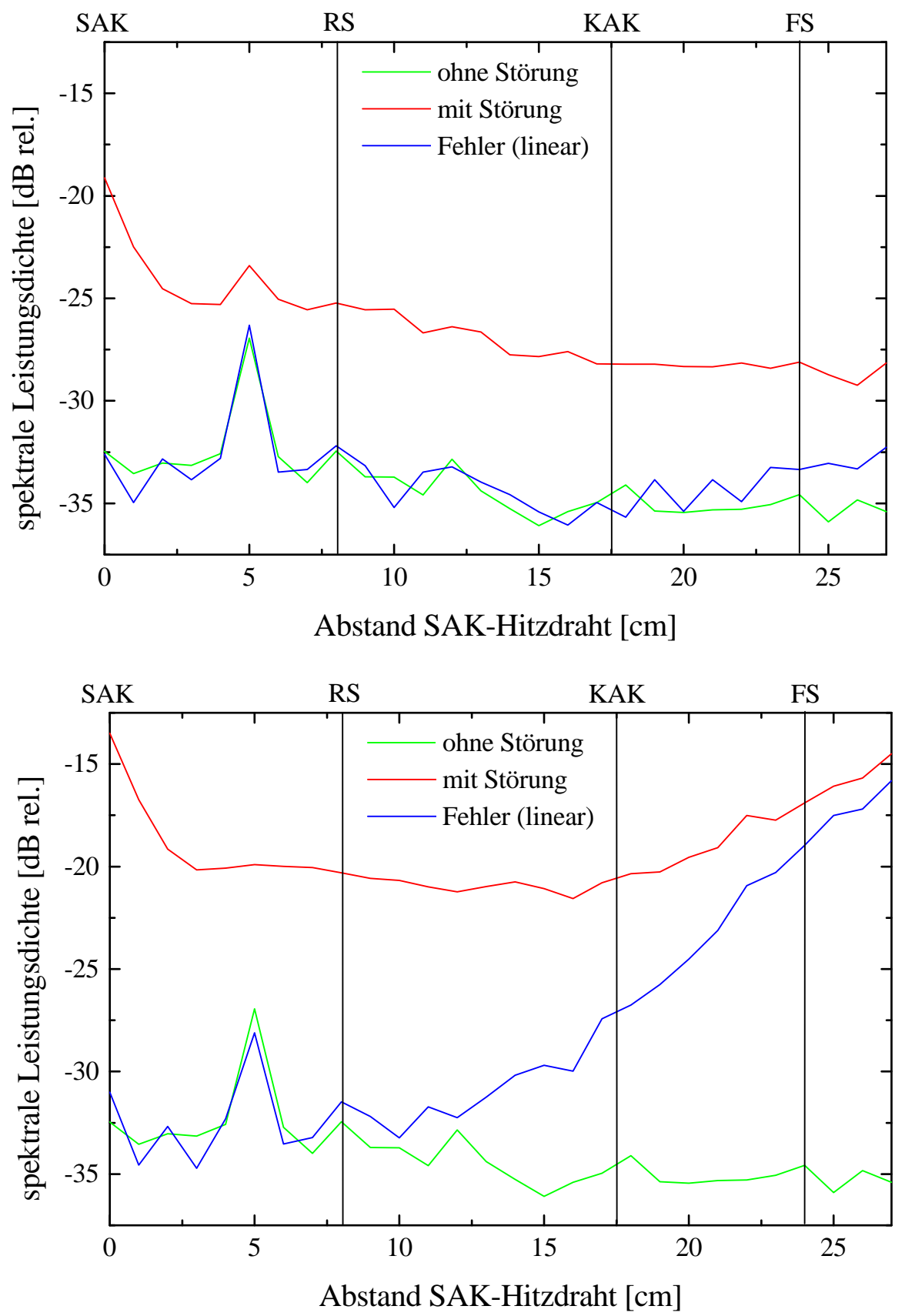

Abbildung 5.22: Spektrale Leistungsdichte des Hitzdrahtsignals (75 Hz) Amplitude: 0,4 (oben) und 0,8 (unten); Traversierung stromab 


\section{Kapitel 6}

\section{Nachbildung nichtlinearer Störausbreitungsprozesse}

Die Ergebnisse des letzten Kapitels haben gezeigt, daß bei der dynamischen Stabilisierung in Abhängigkeit von der Störamplitude auch nichtlineare Ausbreitungsprozesse eine Rolle spielen, wodurch bei größeren Amplituden zunehmend nichtkompensierbare Reststörungen auftreten. Es stellt sich daher die Frage, ob diese Prozesse durch ein nichtlineares Modell nachgebildet werden können, und inwieweit die Verwendung eines solchen Modells zu einer Verbesserung der Störkompensation führen kann. Es wurden daher mit einem Volterra-Filter zunächst Experimente zur Streckenerkennung durchgeführt [9]. Dies bedeutet eine gewisse Vereinfachung gegenüber der dynamischen Stabilisierung, da hier keine Sekundärstreckenintegration erforderlich ist und diese Experimente auch mit gemessenen Zeitreihen offline durchgeführt werden können, wodurch die Echtzeitanforderung wegfällt.

\subsection{Nichtlineare Modellierung mit einem Volterra-Filter}

Eine mögliche Methode zur Untersuchung nichtlinearer Prozesse ist die Messung höherer Korrelationen. Hierauf basiert auch das adaptive Volterra-Filter, das als Potenzreihenentwicklung mit Gedächtnis betrachtet werden kann. 
Das Ausgangssignal $y[n]$ eines allgemeinen kausalen Volterra-Filters ist durch

$$
\begin{aligned}
y[n] & =w_{0}+\sum_{m_{1}=0}^{N-1} w_{m_{1}}^{(1)} x\left[n-m_{1}\right] \\
& +\sum_{m_{1}=0}^{N-1} \sum_{m_{2}=0}^{N-1} w_{m_{1}, m_{2}}^{(2)} x\left[n-m_{1}\right] x\left[n-m_{2}\right] \\
& +\sum_{m_{1}=0}^{N-1} \sum_{m_{2}=0}^{N-1} \cdots \sum_{m_{p}=0}^{N-1} w_{m_{1}, m_{2}, \cdots, m_{p}}^{(p)} x\left[n-m_{1}\right] x\left[n-m_{2}\right] \cdots x\left[n-m_{p}\right]
\end{aligned}
$$

gegeben [26, 32]. Hierbei beschreibt (6.1) einen Gleichanteil und den linearen Anteil (FIR-Filter), (6.2) eine Nichtlinearität zweiter und (6.3) eine Nichtlinearität $p$-ter Ordnung. Der sogenannte Volterra Kern p-ter Ordnung $w_{p}\left[m_{1}, m_{2}, \cdots, m_{p}\right]$ wird im folgenden als symmetrisch, das heißt unabhängig von den $p$ ! möglichen Permutationen der Indizes $m_{1}$ bis $m_{p}$ angenommen.

Betrachtet man als Erweiterung zum linearen Modell zunächst zum Beispiel das adaptive Volterra-Filter zweiter Ordnung, so wird in jedem Zeitschritt das Ausgangssignal $y^{(2)}[n]$ als mit den Filterkoeffizienten $w_{i, j}$ gewichtete Summe der Mischprodukte der letzten $N$ Eingangssamples $x[n]$ berechnet.

$$
y^{(2)}[n]=\sum_{i=0}^{i=N-1} \sum_{j=i}^{j=N-1} w_{i, j}[n] x[n-i] x[n-j]
$$

Obwohl dieses Filter Ausgangssignale erzeugt, welche nichtlinear aus dem Eingangssignal hervorgehen, ist es im Gegensatz z.B. zu neuronalen Netzen linear in den Koeffizienten. Zur optimalen Anpassung der Koeffizienten kann daher wie beim FIR-Filter wieder der LMS-Algorithmus verwendet werden. Die UpdateVorschrift, nach welcher die Koeffizienten adaptiert werden, lautet in diesem Fall

$$
w_{i, j}[n+1]=w_{i, j}[n]+\mu x[n-i] x[n-j] e[n],
$$

für alle unter Ausnutzung der Symmetrieeigenschaft relevanten Mischprodukte bzw. Koeffizienten.

Man sieht, daß hier im Gegensatz zum LMS beim FIR-Filter die Korrelationen zwischen allen möglichen Mischprodukten (zweiter Ordnung) und dem Fehlersignal ausgewertet werden.

Es wurden nun mit dem Referenzsensor und einer Hitzdrahtsonde direkt über dem Kompensationsaktuator Zeitsignale aufgenommen (4 $10^{5}$ Abtaswerte) und nach der eigentlichen Messung im Computer eine Erkennung der Übertragungsprozesse zwischen den beiden Sensoren durchgeführt, wie in Abbildung 3.3 dargestellt. 
Als Anregungsamplitude wurde mit 0,8 die höchste, auch bei den Experimenten zur dynamischen Stabilisierung verwendete Amplitude gewählt. Nach der rein linearen Erkennung mit einem FIR-Filter (80 Koeffizienten) wurde zusätzlich ein Volterra-Filter zweiter Ordnung $(N=80)$ adaptiert. In Abbildung 6.1 ist das Leistungsspektrum des sich als Differenz zwischen Hitzdrahtsignal $d[n]$ und Filterausgangssignal $y[n]$ ergebenden Fehlersignals $e[n]$ für vier verschiedene Fälle dargestellt. Für die Fälle ohne Störung und mit Störung, jedoch ohne Erkennung, entspricht das Fehlersignal dem mit dem Hitzdraht aufgenommenen Signal $d[n]$, da hier noch keine Filterausgabe erfolgt. Wieder zeigt sich eine starke Signalleistung im TS-Grundfrequenzbereich, aber auch die Generierung höherer Frequenzkomponenten. Läßt man nun das Filter erster Ordnung (linear, FIR) adaptieren, so ergibt sich im Grundfrequenzbereich eine gute Erkennung mit einem Restfehler, der allerdings noch um bis zu $15 \mathrm{~dB}$ über dem Grundstörniveau ohne künstliche Anregung liegt. Aber auch für Frequenzen oberhalb von etwa $250 \mathrm{~Hz}$ zeigt sich, daß ein Teil des Hitzdrahtsignals linear mit dem Signal des Referenzsensors korreliert ist, was darauf hinweist, daß auch schon vor dem Referenzsensor Anteile dieses Signals über nichtlineare Ausbreitungsprozesse erzeugt wurden.

Lässt man nicht nur das lineare Filter, sondern die Filter erster und zweiter Ordnung gleichzeitig adaptieren, so zeigt sich neben einer weiteren Reduktion des Fehlers für Frequenzen oberhalb von $250 \mathrm{~Hz}$ auch ein deutlicher Effekt im Grundfrequenzbereich der TS-Instabilität. Hier sinkt die Fehlerleistung im Vergleich zu Erkennung mit einem rein linearen Modell um weitere 4-6 dB. Dies zeigt, daß auch in diesem Frequenzbereich Störkomponenten über nichtlineare Mechanismen generiert werden, deren Übertragungsverhalten mit einem Volterra-Filter nachgebildet werden kann.

Setzt man in einem adaptiven Volterra-Filter mehrere Ordnungen $p$ gleichzeitig ein, so kann man entweder allen Teilfiltern (Ordnungen) das gleiche nachzubildende Signal $d[n]$ (parallel) zur Verfügung stellen, oder man gibt dem Teilfilter der Ordnung $p$ nur noch den Fehler des Filters der Ordnung $p-1$ als zu erkennendes Signal und entkoppelt somit die Teilfilter [14]. Durch die Entkopplung ist es einfacher, die Konvergenzeigenschaften des Filters zu analysieren und Angaben zu den Maximalwerten der Schrittweitenparameter $\mu^{(p)}$ zu machen. Versuche mit entkoppelten Filtern haben jedoch zu schlechteren Nachbildungen mit höheren Restfehlern geführt, weshalb alle hier beschriebenen Experimente mit gekoppelten Filtern durchgeführt wurden.

In Abbildung 6.2 sind oben die Filterkoeffizienten des Filters erster Ordnung dargestellt, und unten die Koeffizientenmatrix des Filters zweiter Ordnung. Hierbei ist in jedem Kästchen farbig kodiert die Korrelation zwischen dem jeweiligen Mischprodukt $x[n-i] x[n-j]$ und dem Hitzdrahtsignal $d[n]$ dargestellt. Vergleicht man die Symmetrieachse $(i=j)$ der Koeffizientenmatrix zweiter Ordnung mit den Filterkoeffizienten des Filters erster Ordnung, so zeigt sich, daß die wesentli- 


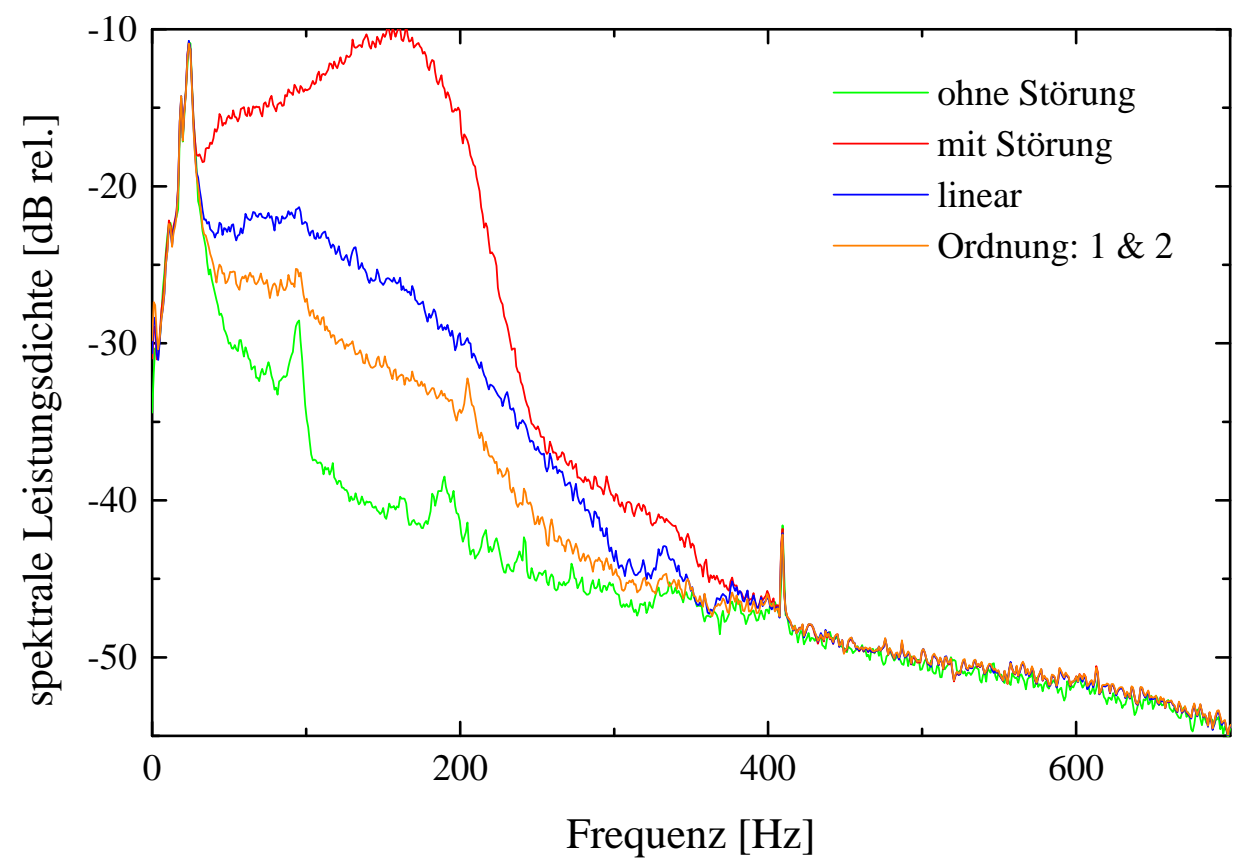

Abbildung 6.1: Spektrale Leistungsdichte des Hitzdrahtsensorsignals ohne und mit Anregung und Fehlersignale nach abgeschlossener Adaption, Amplitude: 0,8

chen Korrelationen im gleichen Zeitbereich konzentriert sind, und daß sogar die Struktur bezüglich der Maxima und Minima sehr ähnlich ist.

Nach diesem Ergebnis stellte sich nun die Frage, inwieweit sich durch eine Erhöhung der Ordnung $p$ des Volterra-Filters eine weitere Verbesserung der Nachbildung erreichen läßt.

Mit zunehmender Ordnung $p$ des Volterrafilters steigt jedoch die Zahl der benötigten Koeffizienten und damit der Rechenaufwand exponentiell an. Unter Berücksichtigung der Symmetrie ergibt sich für die Koeffizientenanzahl $M_{p, N}$ :

$$
M_{p, N}=\left(\begin{array}{c}
N+p-1 \\
p
\end{array}\right)=\frac{(N+p-1) !}{p !(N-1) !}
$$

Bei einer Gedächtnislänge von $N=80$ müßten somit in jedem Zeitschritt für ein Filter dritter Ordnung 88560 Koeffizienten und für die vierte Ordnung zusätzlich $1.8 \cdot 10^{6}$ Koeffizienten berechnet und adaptiert werden. Aus diesem Grund wurde als nächstes versucht, die Anzahl der benötigten Koeffizienten zu reduzieren. Für die dargestellte Streckenerkennung besteht eine erste Möglichkeit offensichtlich in der Vorverzögerung des Eingangssignals $x[n]$, da die anhand von Abb. 6.2 ersichtliche Gruppenlaufzeit von etwa 20-30 Zeitschritten sinnvollerweise nicht von dem Volterramodell nachgebildet werden sollte. 


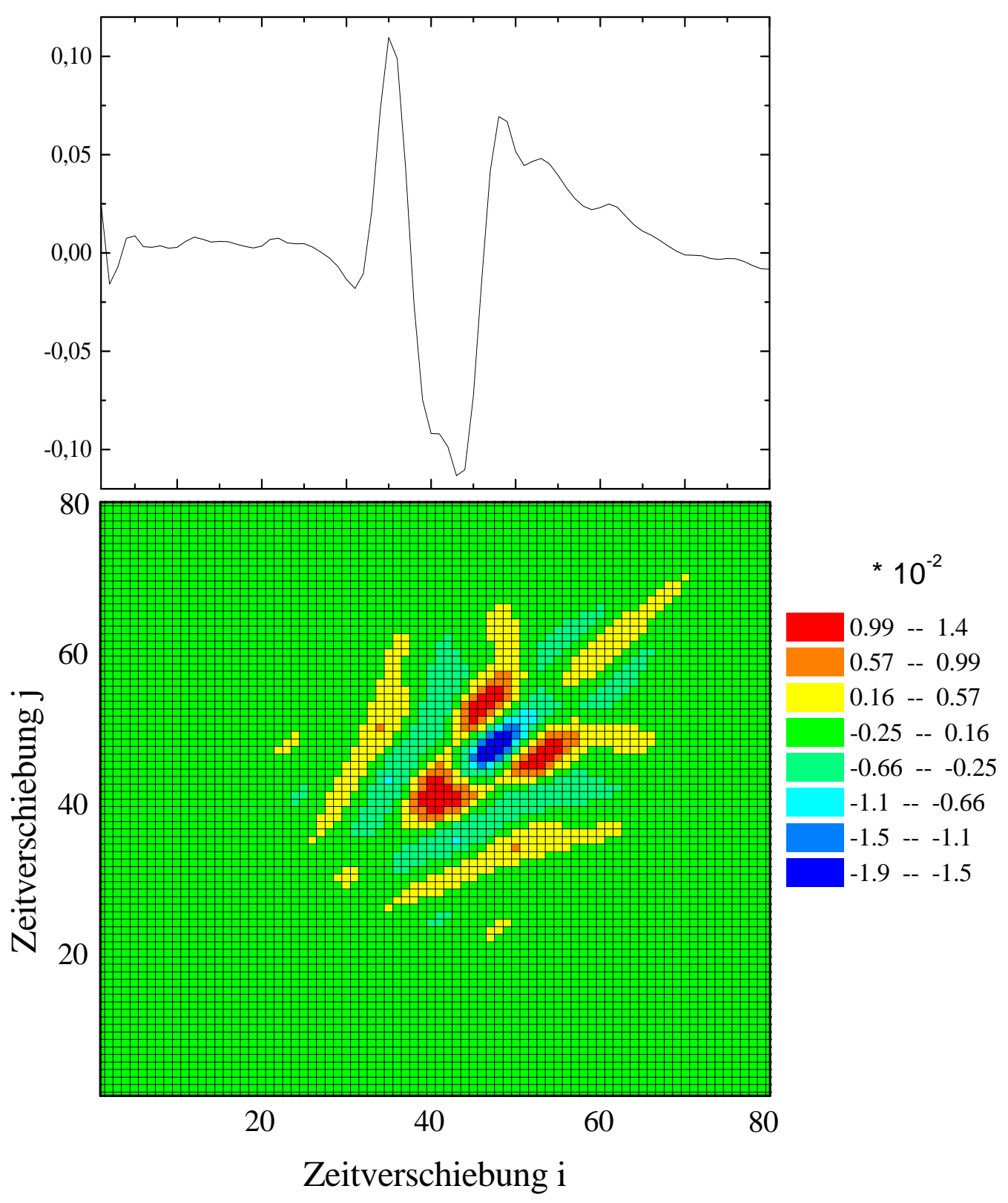

Abbildung 6.2: Koeffizientenvektor des linearen FIR-Filters (oben) und Koeffizientenmatrix des Volterra-Filters zweiter Ordnung (unten), $N=80$, Amplitude: 0,8 
Eine weitere grundsätzliche Möglichkeit besteht darin, wie beim linearen IIRFilter auch in das Volterra-Filter höherer Ordnung einen rekursiven Anteil zu integrieren um dadurch eine Verringerung der notwendigen Koeffizientenanzahl zu erreichen. Verschiedene Arbeiten zeigen jedoch, daß die schon für das lineare IIR-Filter angesprochenen Stabilitätsprobleme noch in viel stärkerem Maße bei Filtern auftreten, die auf einer nichtlinearen, rekursiven Differenzengleichung basieren [26].

In [12] beschreiben Fejzo und Lev-Ari ein sogenanntes Laguerre-Lattice Filter, das bei der Nachbildung von Systemen mit Tief- oder Hochpaßcharakteristik weniger Koeffizienten benötigt, als ein herkömmliches FIR-Filter und verweisen auf die Möglichkeit, ein solches System als eine Art Vorverarbeitung für ein VolterraFilter einzusetzen [11].

Streckenerkennungsexperimente haben jedoch gezeigt, daß ein Laguerre-Filter im Fall der vorliegenden bandpaßartigen Übertragungscharakteristik nicht zu einer Reduktion der Koeffizientenanzahl führt. Will man dieses Vorwissen über die nachzubildende Übertragungsfunktion dennoch von vornherein in die Filterstruktur integrieren, so führt dies zu den sogenannten Kautz-Filtern [25, 6, 5]. Ninnes und Gustafsson zeigen in [28], daß sowohl Laguerre- und Kautz-Filter als auch das FIR-Filter Spezialfälle einer allgemeineren Konstruktionsvorschrift für orthonormale Basen sind.

\subsection{Modellierung mit Vorwissen}

Um den Unterschied zwischen einem Kautz-Filter und einem FIR-Filter deutlich zu machen, sind die Filterstrukturen in Abb. 6.3 und Abb. 6.4 dargestellt. Beim FIR-Filter wird das Eingangssignal zunächst auf $N$ einzelne Kanäle aufgeteilt, wobei sich die Signale in diesen Kanälen dadurch unterscheiden, daß sie jeweils um einen Zeitschritt gegeneinander verschoben sind $\left(z^{-1}\right.$ : Verzögerung um einen Zeitschritt). Diese Signale werden dann mit den Filterkoeffizienten gewichtet und zum Ausgangssignal $y[n]$ aufsummiert.

Betrachtet man das Kautz-Filter, so zeigt sich, daß es sich vom FIR-Filter nur in der Vorverarbeitung des Eingangssignals bei der Bildung der Signale für die einzelnen Kanäle unterscheidet. Anstelle einfacher Verzögerungsoperatoren finden sich hier Bandpaß-, Allpaß- und Tiefpaßfilter. Der verwendete Bandpaß ist ein Resonator mit einem komplex konjugierten Polpaar. Seine Übertragungsfunktion lautet:

$$
B P(z)=\frac{\sqrt{1-c^{2}}(z-b)}{z^{2}+b(c-1) z-c}
$$




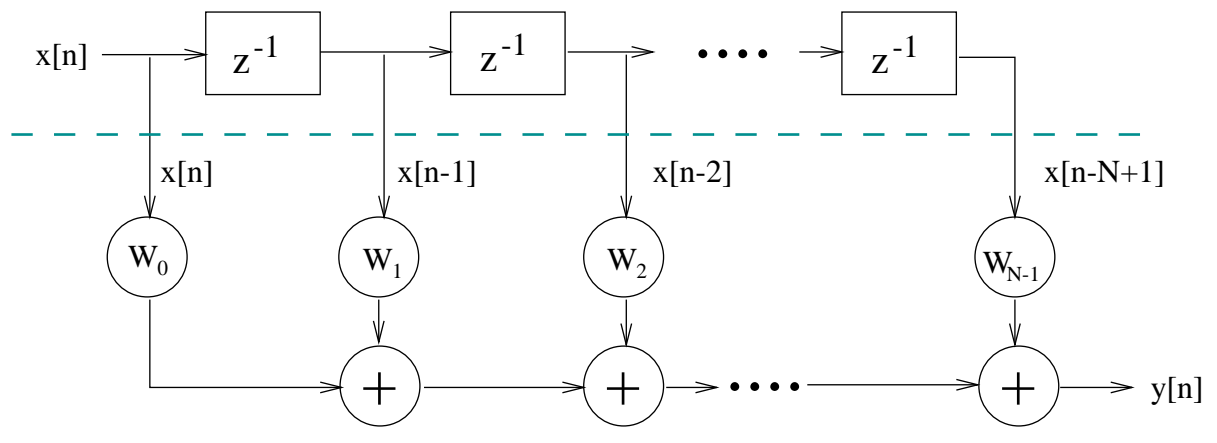

Abbildung 6.3: Struktur eines FIR-Filters

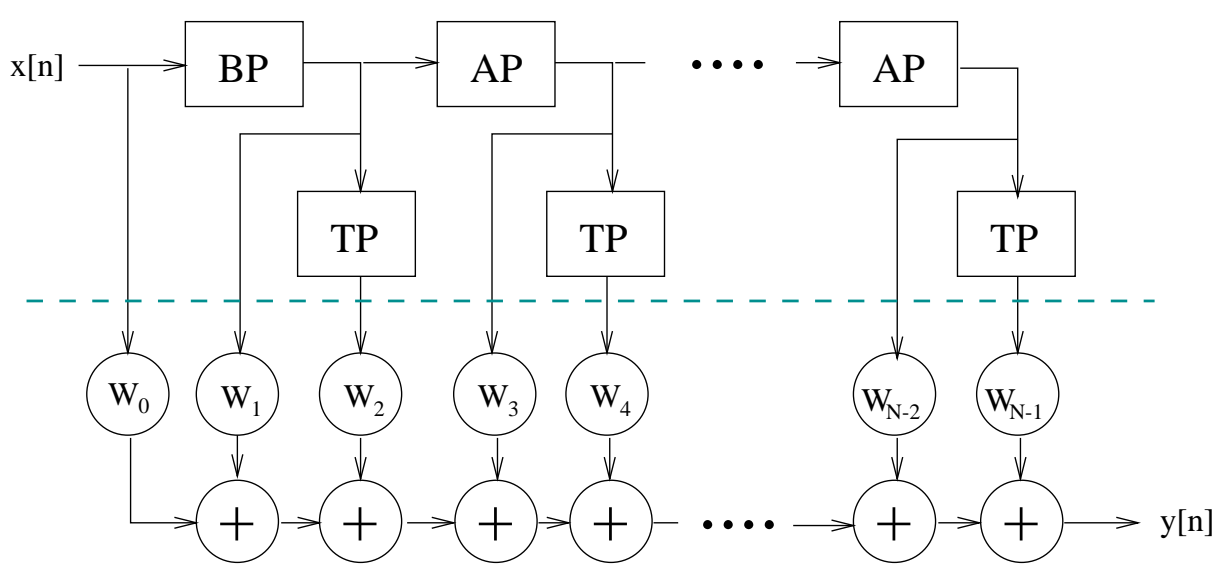

Abbildung 6.4: Struktur eines Kautz-Filters 
Die Übertragungsfunktionen der Allpässe und Tiefpässe sind durch

$$
A P(z)=\frac{-c z^{2}+b(c-1) z+1}{z^{2}+b(c-1) z-c}
$$

und

$$
T P(z)=\frac{\sqrt{1-b^{2}}}{z-b}
$$

bestimmt.

Die Lage des Polpaars und damit das Durchlaßfrequenzband muß durch eine geeignete Wahl der Parameter $b$ und $c$ anhand des vorhandenen Vorwissens über das nachzubildende System festgelegt werden. Gibt man die Resonanzfrequenz $f_{R}$ und den Betrag des Polpaars $\left|z_{*}\right|$ in der z-Ebene vor, so lassen sich die Parameter hieraus leicht berechnen. Werden $b$ und $c$ gleich Null gesetzt, so vereinfachen sich $B P(z)$ und $T P(z) \mathrm{zu}^{-1}$ und $A P(z) \mathrm{zu} z^{-2}$, wodurch man wieder das FIR-Filter erhält.

In Abbildung 6.5 ist das Leistungsspektrum des Fehlers einer Streckenerkennung mit einem FIR-Filter und einem Kautz-Filter dargestellt. Es wurde wieder die Strecke zwischen dem Referenzsensor und dem Hitzdrahtsensor über dem Kompensationsaktuator nachgebildet. Mit 0,2 wurde hier jedoch eine Störamplitude verwendet, bei der noch keine wesentlichen nichtlinearen Prozesse zu erwarten sind. Für beide Filter wurden 80 Koeffizienten verwendet und es zeigt sich in beiden Fällen nach abgeschlossener Adaption ein geringer Restfehler, der im wesentlichen mit dem Hitzdrahtsignal ohne künstlich eingebrachte Störung identisch ist.

Interessant ist ein Vergleich der sich einstellenden Filterkoeffizienten (Abb. 6.6). Hier sieht man, daß sich die wesentlichen Korrelationen im Fall des Kautz-Filters über deutlich weniger Koeffizienten erstrecken als beim FIR-Filter.

Dieses Ergebnis wurde mit optimalen Parametern für die Resonanzfrequenz $\left(f_{R}=\right.$ $130 \mathrm{~Hz})$ und den Betrag $\left(\left|z_{*}\right|=0,75\right)$ erzielt.

Um diese optimalen Parameter zu finden und einen Eindruck von der Sensitivität des Systems bezüglich nicht optimaler Parameter zu bekommen, wurde die Streckenerkennung für einen großen Parameterbereich durchgeführt und dabei, nach abgeschlossener Adaption, jeweils der mittlere quadratische Fehler (mean square error, MSE) bestimmt. Um eine deutliche Abhängigkeit von den Parametern Resonanzfrequenz und Betrag des Polpaars in der z-Ebene zu erreichen, wurden nur zwanzig Koeffizienten verwendet. Das Ergebnis dieser Untersuchung ist in Abbildung 6.7 dargestellt.

Es zeigt sich ein globales Minimum bei $(130 ; 0,75)$. Betrachtet man die Übertragungsfunktion (Abb. 6.8), die sich als Fouriertransformierte der Koeffizienten 


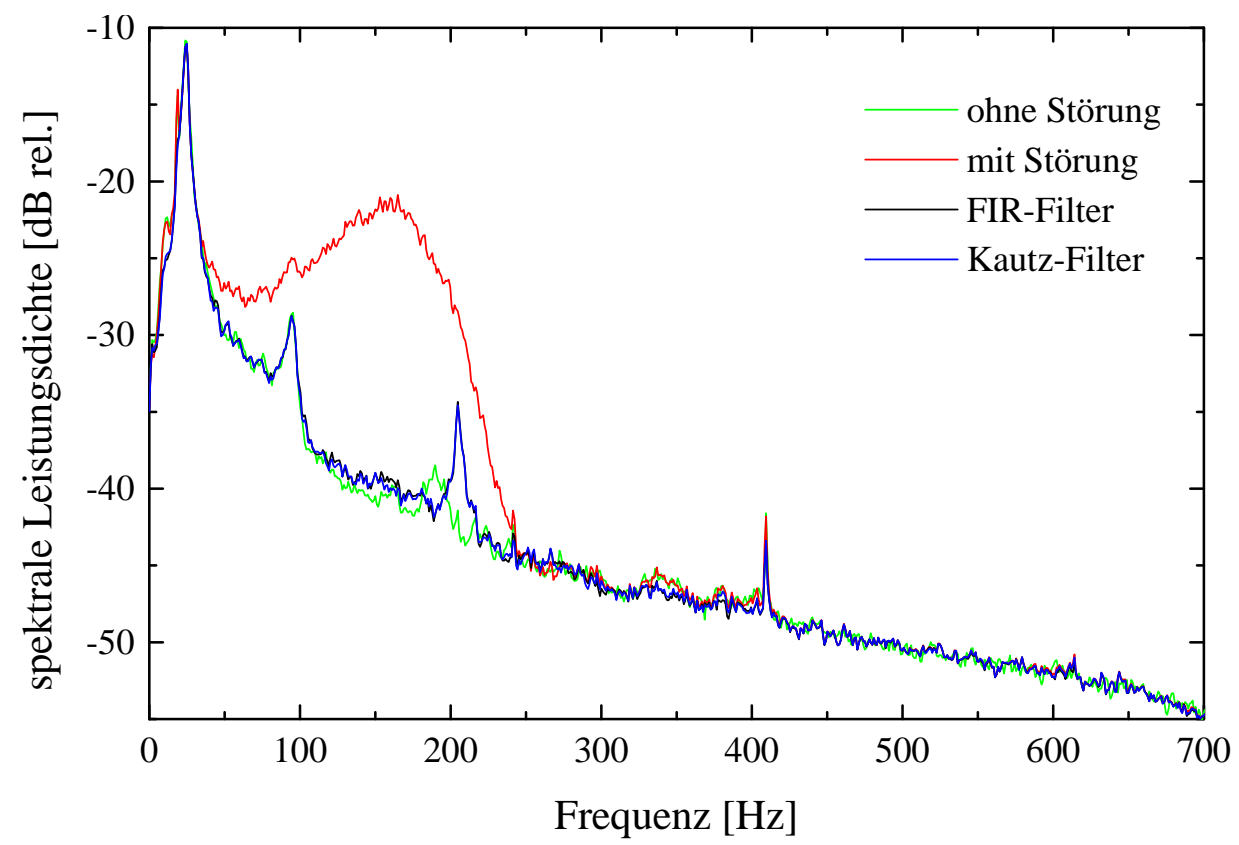

Abbildung 6.5: Spektrale Leistungsdichte des Fehlersignals aus einer Streckenerkennung mit einem FIR-Filter und einem Kautz-Filter, $N=80$, Amplitude: 0,2

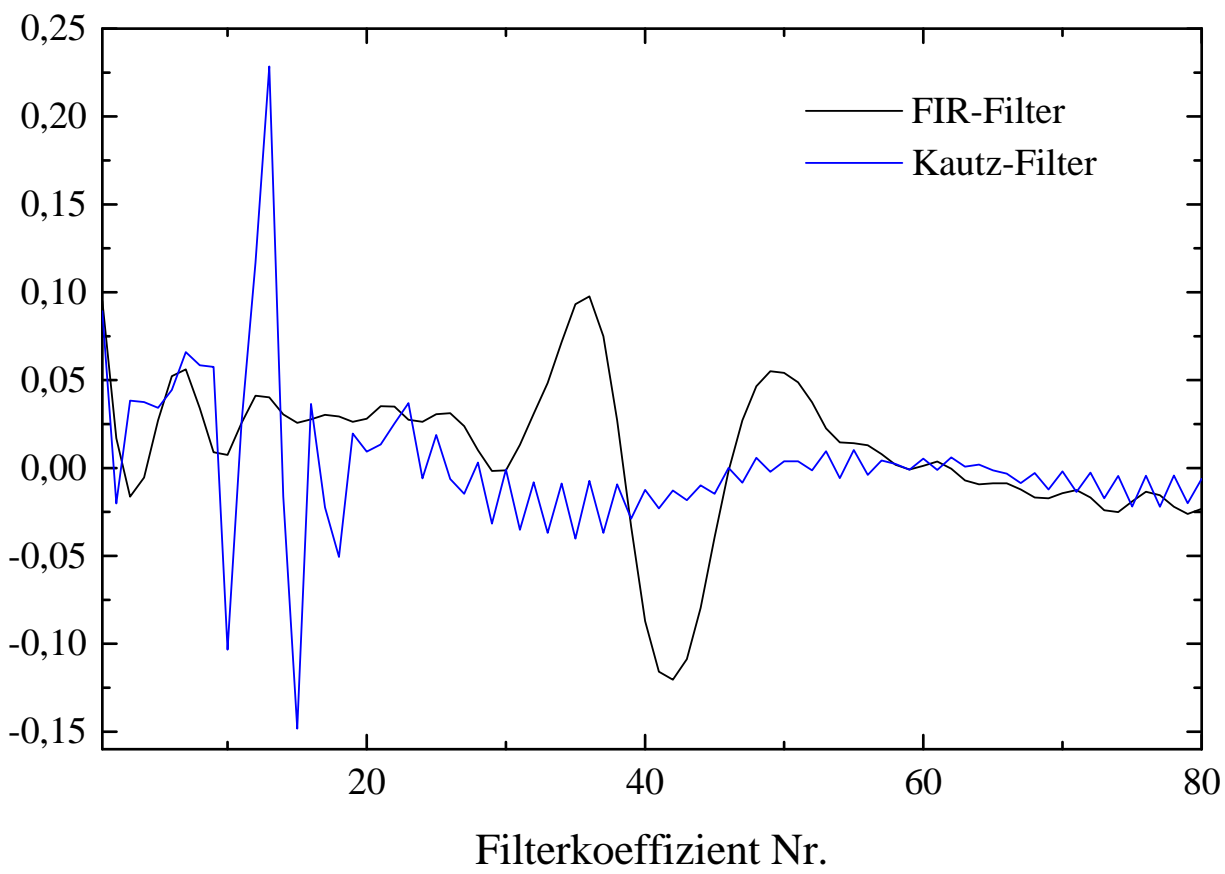

Abbildung 6.6: Vergleich der Koeffizientenvektoren 


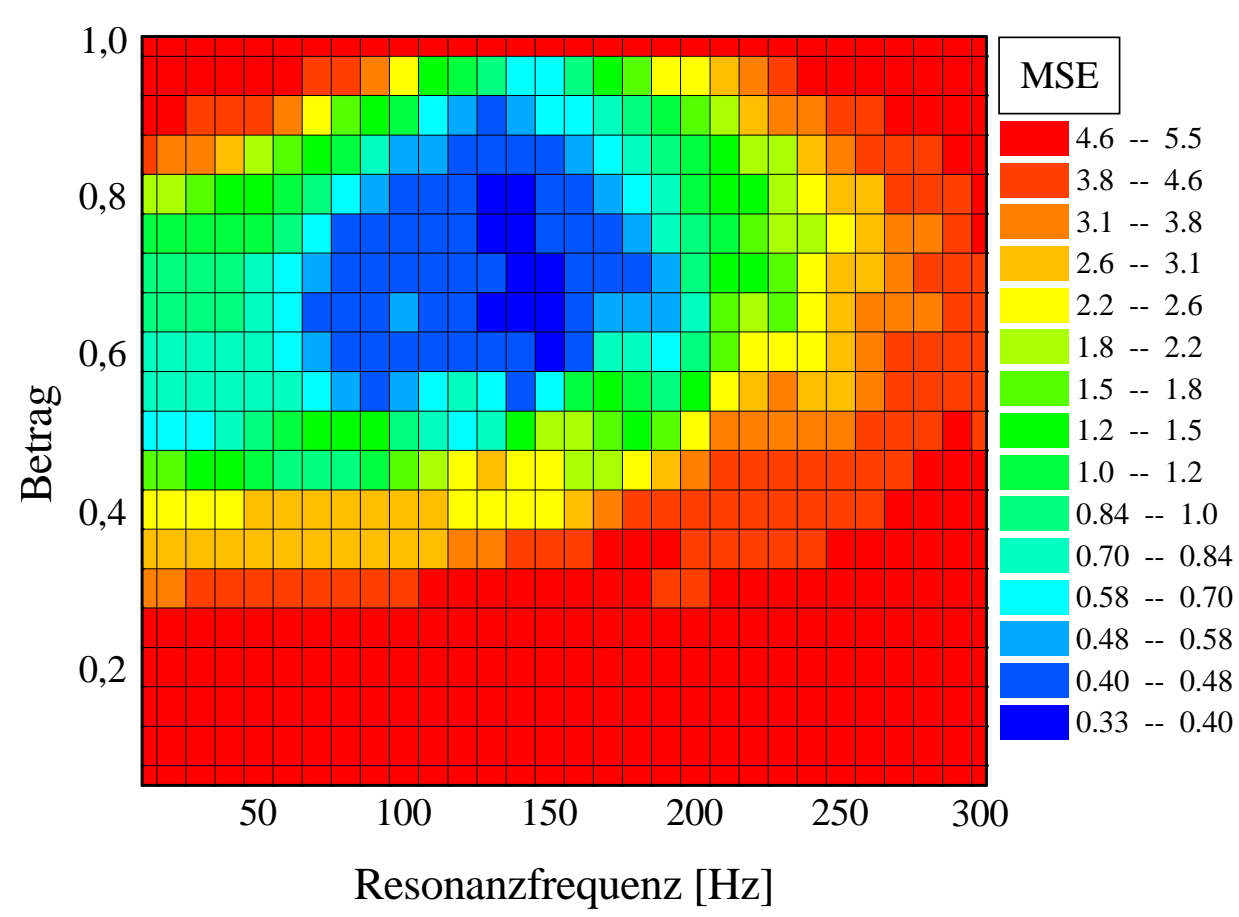

Abbildung 6.7: Mittlerer quadratischer Fehler (MSE) nach Adaption des KautzFilters als Funktion des Betrages und der Resonanzfrequenz des Filters, $N=20$

des FIR-Filters (Abb. 6.6) ergibt, so zeigt sich, daß bei der optimalen Resonanzfrequenz für das komplex-konjugierte Polpaar gerade das Maximum dieser Übertragungsfunktion liegt.

In der Praxis könnte die Lage des Polpaars bezüglich der Frequenz anhand des Instabilitätsdiagramms (Abb. 4.4) abgeschätzt werden. Der Betrag sollte dann auch adaptiv optimiert werden, wie es in [3] für den einen freien Parameter des Laguerre-Filters vorgeschlagen wird.

Abbildung 6.6 gibt zwar einen ersten Eindruck über eine mögliche Reduktion der Koeffizientenanzahl bei Verwendung des Kautz-Filters, aber eine wirkliche Beurteilung kann nur anhand des Fehlers nach abgeschlossener Adaption als Funktion der Koeffizientenanzahl $N$ vorgenommen werden. Daher wurde die Streckenerkennung mit beiden Modellen für unterschiedliche Koeffizientenanzahlen $(N=1$ bis $N=70$ ) durchgeführt und jeweils das Leistungsspektrum des Fehlers berechnet. Das Ergebnis ist in Abbildung 6.9 für das FIR-Filter und in Abbildung 6.10 für das Kautz-Filter dargestellt. Die starke spektrale Leistung bei etwa 20 $\mathrm{Hz}$ (rot) resultiert im wesentlichen aus der Sondenschwingung und bleibt daher unabhängig von der Koeffizientenanzahl erhalten, da dieser Signalanteil nicht mit dem Signal des Referenzsensors (Oberflächenhitzdraht) korreliert ist.

Betrachtet man die Ergebnisse für das FIR-Filter (Abb. 6.9) bis zur Koeffizienten- 


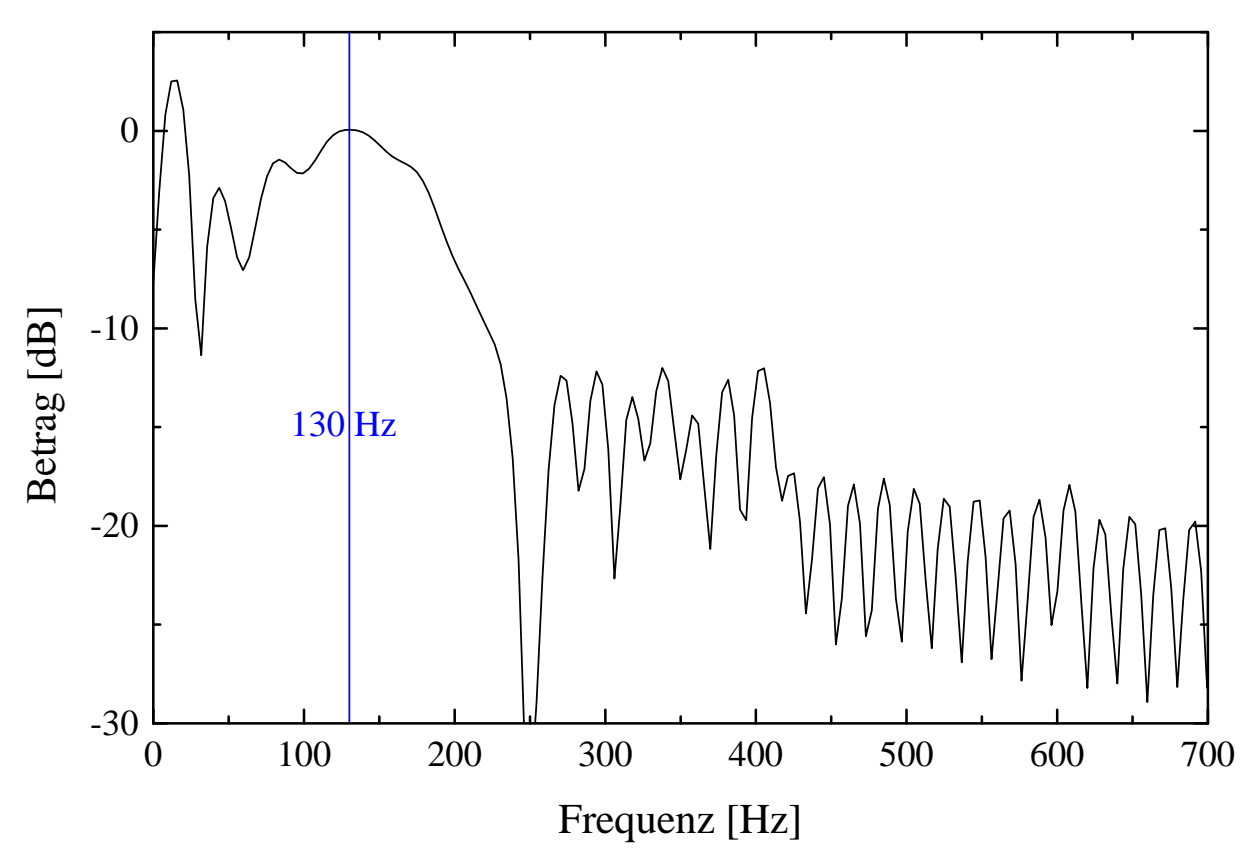

Abbildung 6.8: Betrag der Übertragungsfunktion berechnet aus den Koeffizienten des FIR-Filters

anzahl $N=25$, so sieht man wieder eine starke spektrale Anhebung zwischen etwa $100 \mathrm{~Hz}$ und $220 \mathrm{~Hz}$. Bis zu dieser Koeffizientenanzahl ist durch das FIR-Filter keine wesentliche Nachbildung und damit Unterdrückung dieser Frequenzkomponenten im Fehlersignal zu erkennen. Erst mit etwa 40 Koeffizienten ist eine solche Nachbildung vollständig möglich, wie an der deutlichen Reduktion des Fehlers erkennbar ist. Mit dem Kautz-Filter ist eine solche Fehlerreduktion schon mit etwa 15 Koeffizienten gegeben, was einer Verringerung der Koeffizientenanzahl um den Faktor 2,6 entspricht.

Wie schon in Abschnitt 6.1 angesprochen, könnte eine Vorverzögerung des Eingangssignals hinsichtlich der nichtlinearen Modellierung zu einer deutlichen Aufwandsreduktion führen. Um zu überprüfen, ob eine solche Vorverzögerung die eben gezeigten Ergebnisse zugunsten des FIR-Filters verschieben würden, wurden die gleichen Rechnungen erneut mit einem um 20 Zeitschritte verzögerten Referenzsignal, durchgeführt. Da sich beim FIR-Filter die Signale zwischen zwei benachbarten Koeffizienten gerade durch die Verzögerung um einen Zeitschritt $\left(z^{-1}\right)$ ergeben, reduziert sich hierdurch die Anzahl der benötigten Koeffizienten um etwa 20 auf $N=20$.

Aber auch für das Kautz-Filter ergibt sich eine Reduktion der benötigten Koeffizientenanzahl auf etwa $N=8$, so daß sich an dem Vorsprung des Kautz-Filters hinsichtlich der Koeffizientenanzahl nichts ändert. Diese Reduktion der Koeffizientenanzahl führt allerdings nicht unbedingt zu einer Rechenzeitersparnis, da 


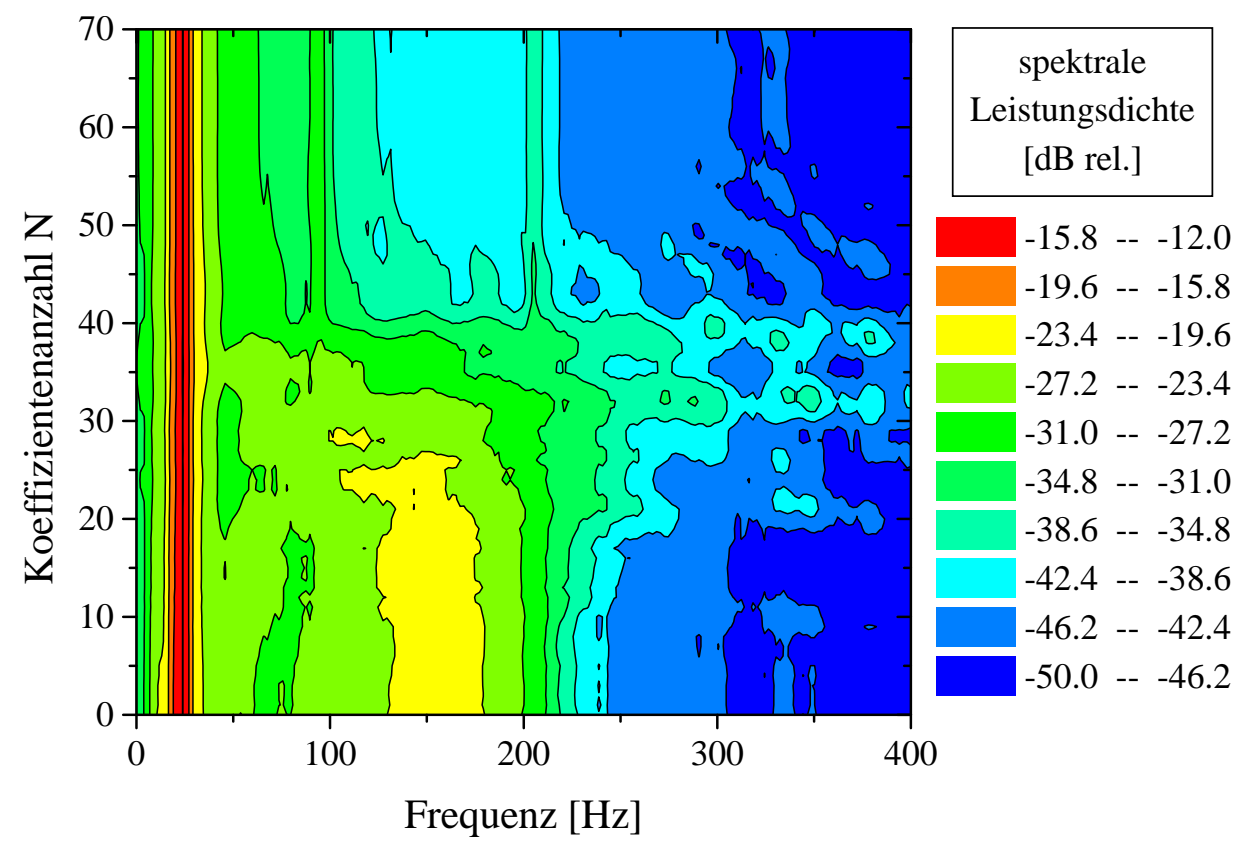

Abbildung 6.9: Spektrale Leistungsdichte des Fehlersignals aus einer Streckenerkennung mit einem FIR-Filter als Funktion der Koeffizientenanzahl $N$

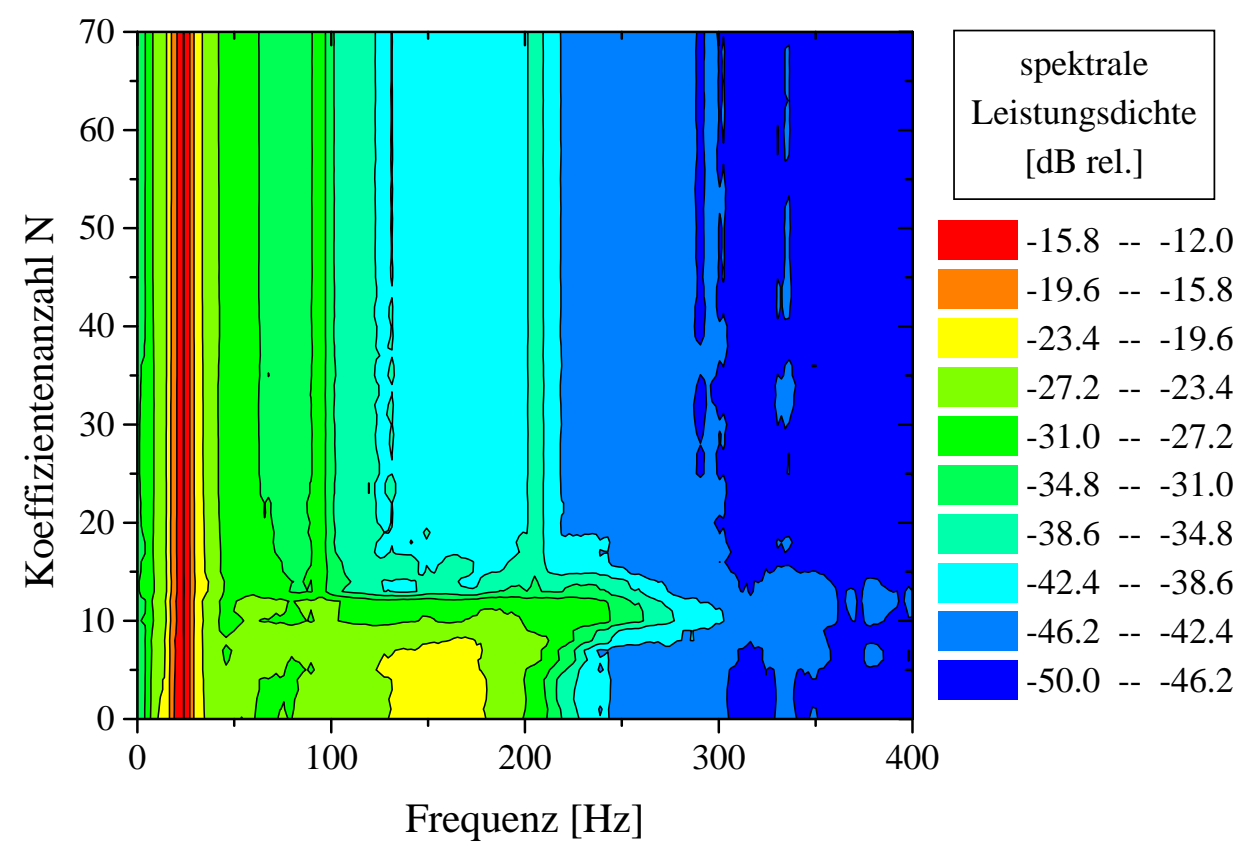

Abbildung 6.10: Spektrale Leistungsdichte des Fehlersignals aus einer Streckenerkennung mit einem Kautz-Filter als Funktion der Koeffizientenanzahl $N$ 


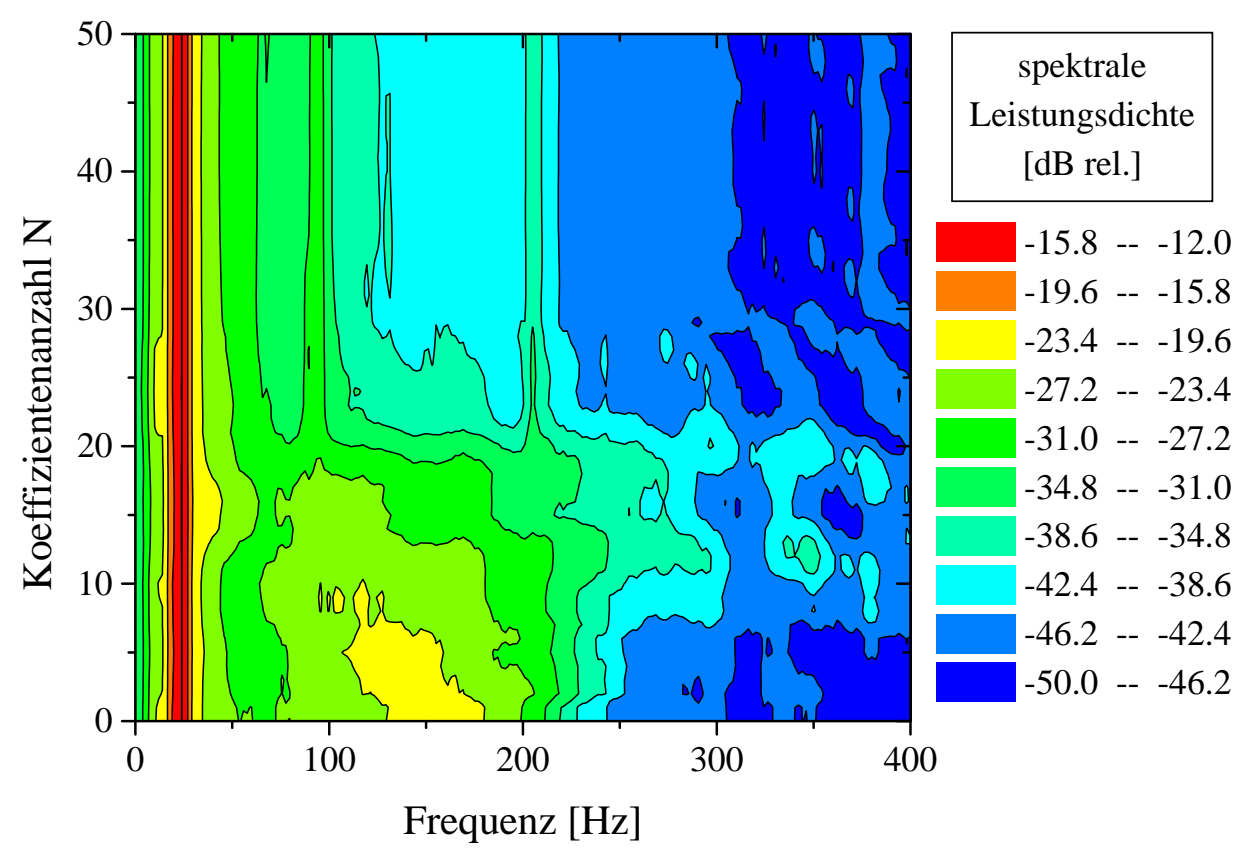

Abbildung 6.11: Spektrale Leistungsdichte des Fehlersignals aus einer Streckenerkennung mit einem FIR-Filter als Funktion der Koeffizientenanzahl $N$ und einer Vorverzögerung um 20 Sample

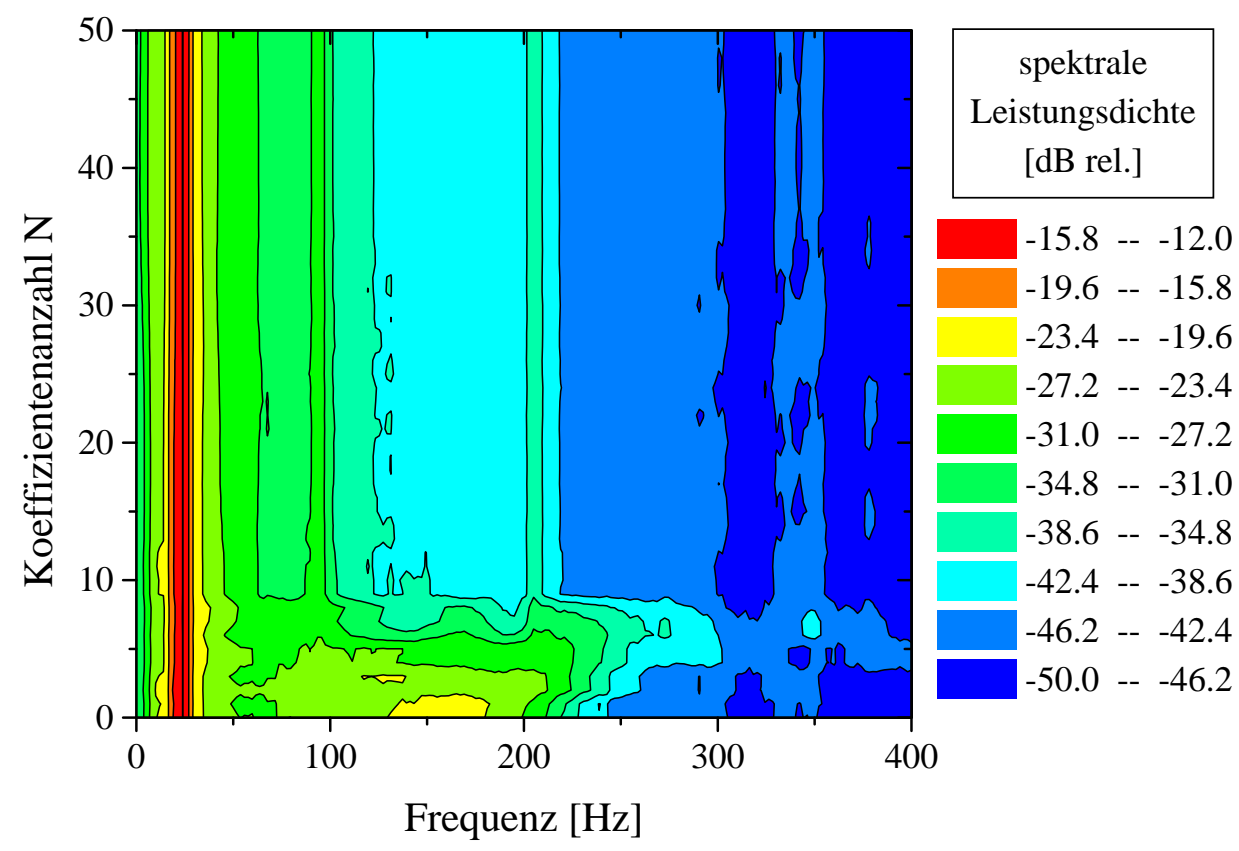

Abbildung 6.12: Spektrale Leistungsdichte des Fehlersignals aus einer Streckenerkennung mit einem Kautz-Filter als Funktion der Koeffizientenanzahl $N$ und einer Vorverzögerung um 20 Sample 
beim Kautz-Filter eine komplexere Vorverarbeitung nötig ist. Es läßt sich zeigen, daß für das adaptive Kautz-Filter pro Koeffizient etwa 2,4 mal soviele Rechenoperationen (Multiplikationen und Additionen) auszuführen sind, wie bei einem FIR-Filter. Viel interessanter ist jedoch die Möglichkeit, ein solches Kautz-Filter über die Mischproduktbildung zwischen den einzelnen Kanälen zu einem nichtlinearen Modell zu erweitern. Ein solcher „Polynom-Generator" wäre in Abbildung 6.4 an der gestrichelten Linie zwischen die Vorverarbeitung und die Koeffizientenwichtung einzufügen. Ist auch im nichtlinearen Fall die Mischproduktbildung aus nur noch etwa halb so vielen vorverarbeiteten Signalen notwendig, führt dieses Verfahren insbesondere für höhere Filterordnungen zu einer deutlichen Reduktion des Rechenaufwandes.

\subsection{Nichtlineares Kautz-Filter}

Nachdem sich gezeigt hatte, daß ein Kautz-Filter zur Nachbildung der linearen Korrelationen deutlich weniger Koeffizienten benötigt als ein FIR-Filter, wurde untersucht, inwieweit diese Aussage auch für Korrelationen höherer Ordnung gültig ist [10]. Für die folgenden Streckenerkennungsexperimente wurden die gleichen Signale (Amplitude: 0,8) verwendet, wie bei den Untersuchungen mit einem Volterra-Filter (s. Abschnitt 6.1). Zunächst wurde eine Erkennung mit einem Modell erster und zweiter Ordnung durchgeführt, wobei $N=80$ gesetzt wurde, um einen direkten Vergleich mit den Ergebnissen des Volterra-Filters zu ermöglichen. Vergleicht man die in Abbildung 6.13 dargestellten Koeffizienten des nichtlinearen Kautz-Filters mit den Ergebnissen für das Volterra-Filter (Abb. 6.2), so zeigt sich beim Kautz-Filter sowohl für den linearen Anteil aber insbesondere auch für die zweite Ordnung eine wesentlich kompaktere Kodierung der Korrelationen mit deutlich weniger Koeffizienten.

Durch die auch im nichtlinearen Fall erreichte Koeffizientenersparnis konnte das adaptive Modell bei vertretbarem Rechenaufwand nun auch zur Untersuchung noch höherer Korrelationen verwendet werden. Durch eine zusätzliche Vorverzögerung des Referenzsignals um 25 Zeitschritte war es möglich, die wesentlichen Korrelationen p-ter Ordnung mit $M_{p, N=15}$ Koeffizienten darzustellen. Im Fall der ersten und zweiten Ordnung ergeben sich die in Abb. 6.14 dargestellten Koeffizienten.

Die spektrale Leistungsdichte des Fehlersignals für eine Modellierung mit nichtlinearen Kautz-Filtern bis zur vierten Ordnung ist in Abbildung 6.15 aufgetragen. Im Grundfrequenzbereich und insbesondere auch für die tiefen Frequenzen zwischen $50 \mathrm{~Hz}$ und $100 \mathrm{~Hz}$ zeigt sich im Vergleich mit der linearen Erkennung eine zusätzliche Reduktion des Fehlers um bis zu $8 \mathrm{~dB}$ durch die nichtlineare Modellierung. Interessant ist hierbei, daß mit dem linearen Kautz-Filter (blau) keine Erkennung der Signalkomponenten im Bereich um $300 \mathrm{~Hz}$ erreicht wird, obwohl 


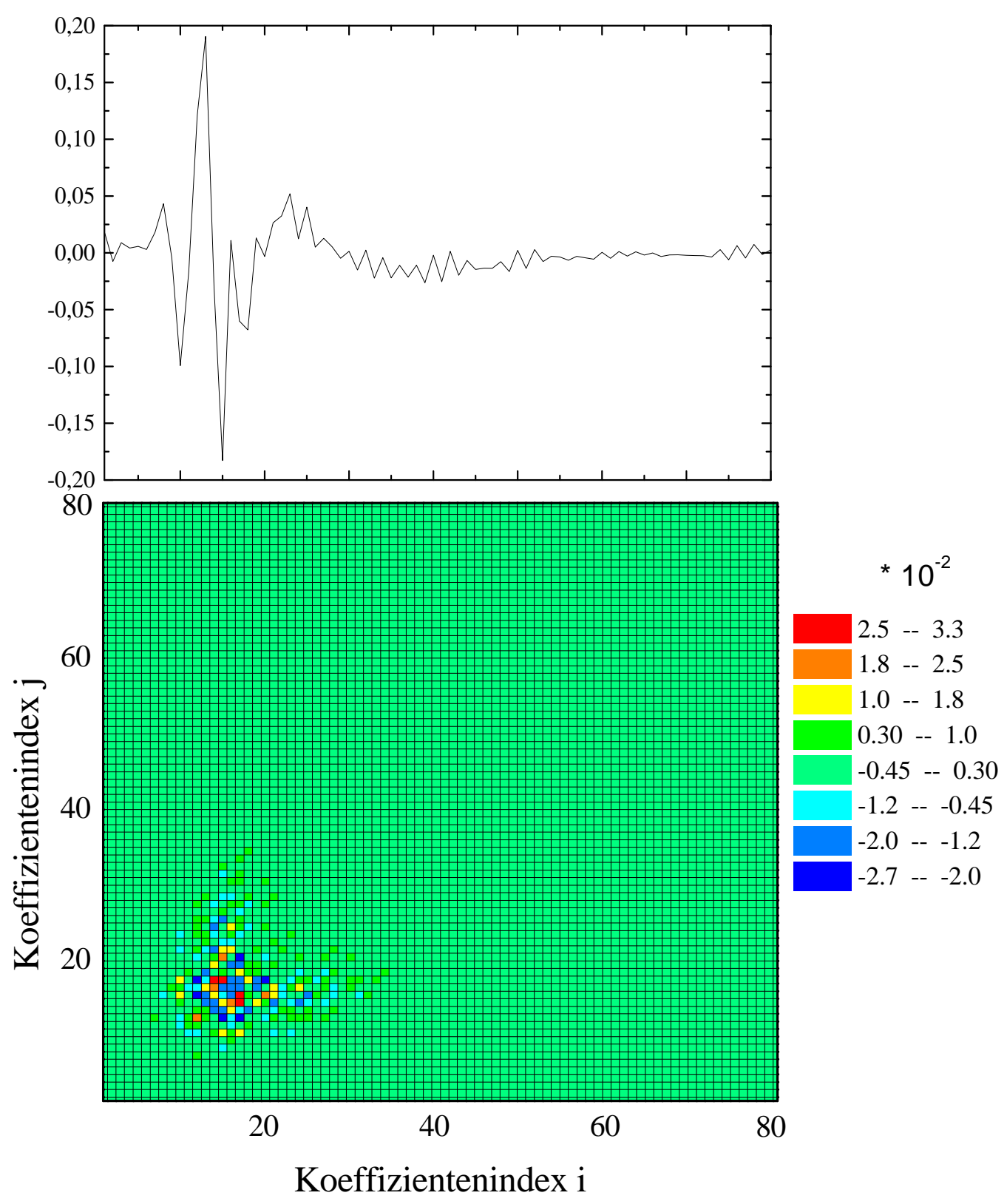

Abbildung 6.13: Koeffizientenvektor des linearen Kautz-Filters (oben) und Koeffizientenmatrix des Kautz-Filters zweiter Ordnung (unten), $N=80$, Amplitude: 0,8 


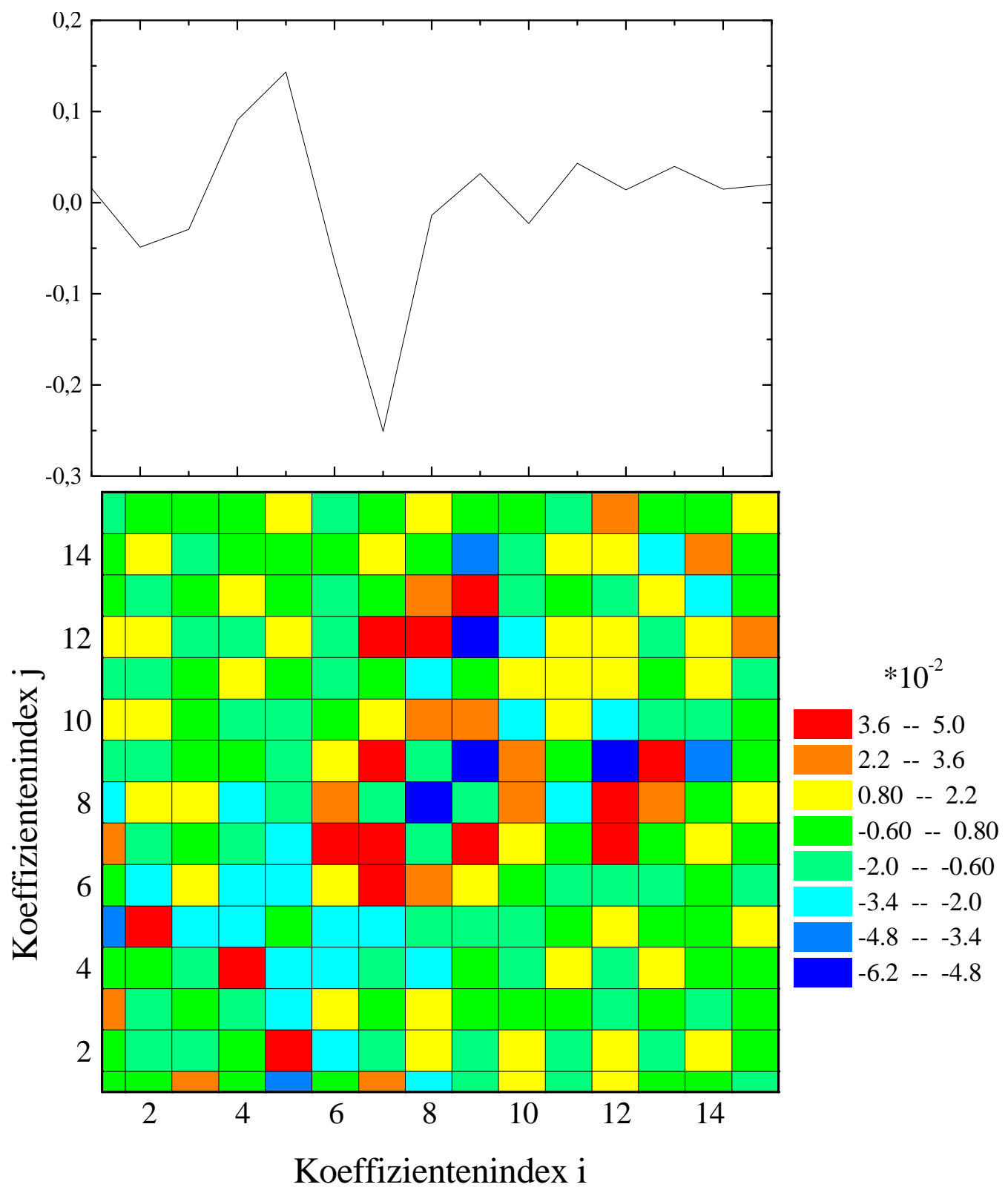

Abbildung 6.14: Koeffizientenvektor des linearen Kautz-Filters (oben) und Koeffizientenmatrix des Kautz-Filters zweiter Ordnung (unten), $N=15$ 


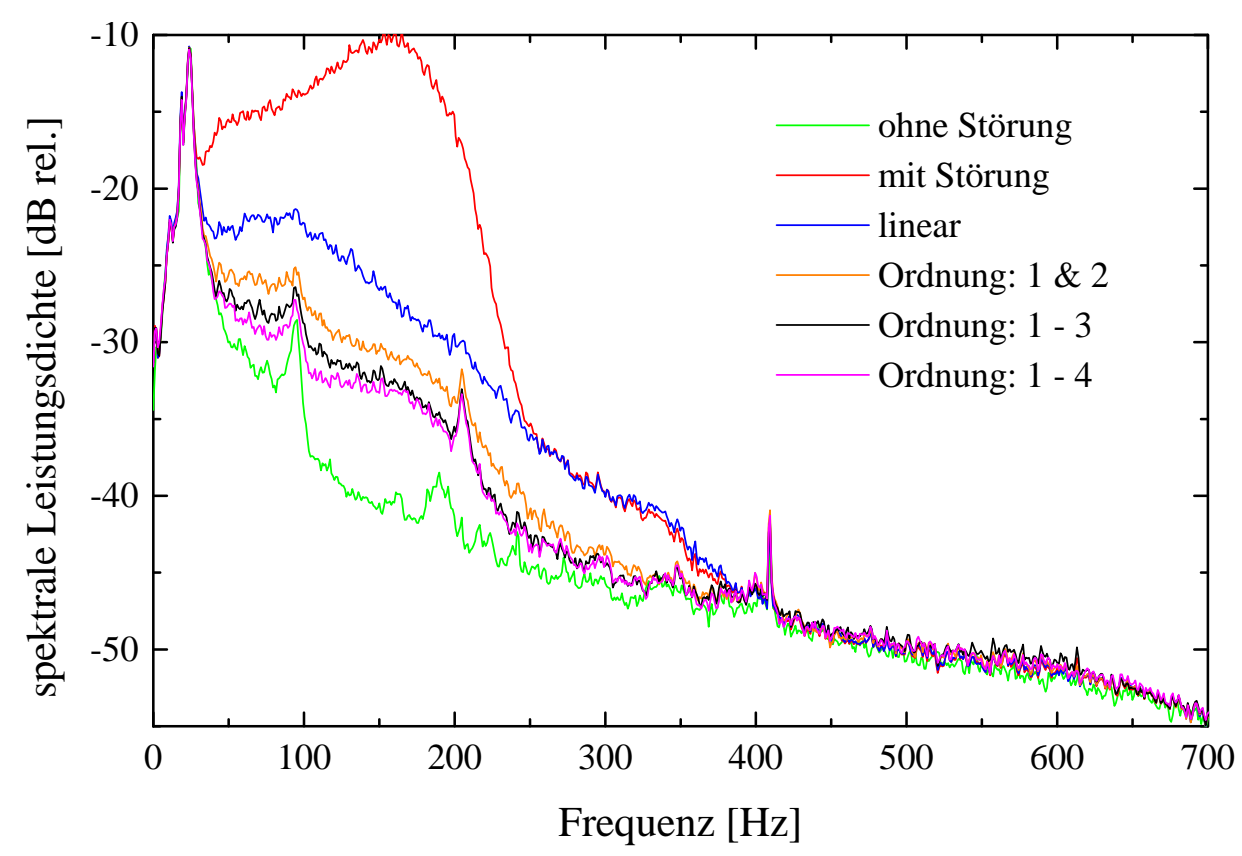

Abbildung 6.15: Spektrale Leistungsdichte des Fehlers der Streckenerkennung, $N=15$, Amplitude: 0,8

sich bei der Nachbildung mit einem Volterra-Filter gezeigt hat, daß diese Signalanteile teilweise linear mit dem Referenzsignal korreliert sind (s. Abb 6.1). Dies läßt sich darauf zurückführen, daß für die lineare Nachbildung dieser höherfrequenten Signalanteile $N=15$ Koeffizienten nicht ausreichen, da die gewählte Resonanzfrequenf $f_{R}$ des Kautz-Filters mit $130 \mathrm{~Hz}$ deutlich niedriger liegt. 


\section{Kapitel 7}

\section{Dynamische Stabilisierung (nichtlineares Modell)}

Nachdem sich gezeigt hatte, daß es möglich ist, die auftretenden nichtlinearen Ausbreitungsprozesse in der Grenzschicht zumindest teilweise nachzubilden, wurden erste Experimente zur dynamischen Stabilisierung mit einem Volterra-Filter erster und zweiter Ordnung durchgeführt.

Zur Adaption des nichtlinearen Filters (zweite Ordnung) kann nicht mehr der filtered-x LMS Algorithmus verwendet werden, da für dessen Herleitung die Linearität und damit Vertauschbarkeit der einzelnen Filteroperatoren vorausgesetzt werden muß (s. Anhang A). Eine andere Möglichkeit, den richtigen zeitlichen Zusammenhang zwischen dem Referenzsignal und dem Fehlersignal wiederherzustellen, ist die Filterung des Fehlersignals mit einer Schätzung der inversen Sekundärstrecke [19, 36]. Hierbei wird davon ausgegangen, daß es ein kausales Filter $\widehat{S}(z)^{-1}$ gibt, das zusammen mit der Sekundärstrecke $S(z)=A_{2}(z) S^{\prime}(z) B_{2}(z)$ eine reine Verzögerung um $k$ Zeitschritte darstellt

$$
\widehat{S}(z)^{-1} S(z)=z^{-k}
$$

Das Prinzip der inversen Sekundärstreckenerkennung ist in Abbildung 7.1 dargestellt.

Um wieviele Zeitschritte $k$ das desired-Signal mindestens zu verzögern ist, um die wesentlichen Anteile der inversen Sekundärstrecke mit dem kausalen Filter $W(z)$ darzustellen, kann mittels einer ersten Nachbildung mit einer großen Verzögerung, anhand der sich einstellenden Impulsantwort relativ leicht abgeschätzt werden. Allgemein müßte auch für die Sekundärstrecke, und damit für ihre Inverse, ein nichtlineares Modell zugrunde gelegt werden, jedoch wurde hier aufgrund der in den Experimenten erreichten guten Kompensation und damit vergleichsweise geringen Störamplitude hinter dem Kompensationsaktuator, eine lineare Schätzung 


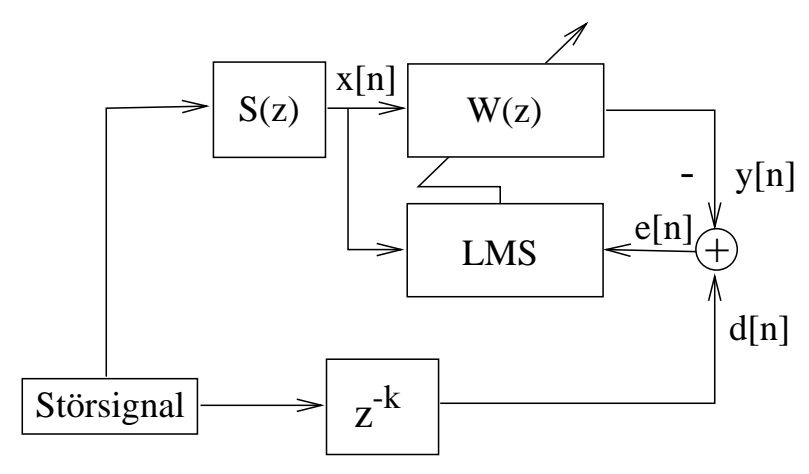

Abbildung 7.1: Prinzip der inversen Sekundärstreckenerkennung

der Inversen als ausreichend erachtet.

Für die Anpassung des linearen Filters (erste Ordnung) wurde weiterhin der filtered-x LMS Algorithmus verwendet, so daß sich für die Signalverarbeitung der in Abbildung 7.2 dargestellte Aufbau ergibt.

Als Störamplitude wurde mit 0,8 die höchste bei den bisher vorgestellten Untersuchungen verwendete Amplitude gewählt. Im ersten Experiment wurde, wie auch bei den Untersuchungen zur dynamischen Stabilisierung mit einem linearen Modell, der Oberflächenhitzdraht als Fehlersensor benutzt.

In Abbildung 7.3 ist die spektrale Leistungsdichte des Fehlersignals zusätzlich zu den schon aus den Experimenten zur dynamischen Stabilisierung mit einem linearen Modell bekannten Fällen, ohne Störung (grün), mit Störung (rot) und mit Kompensation (hier linear, blau) für die Kompensation unter Verwendung des Filters erster und zweiter Ordnung (orange) dargestellt. Auf eine separate Messung des Kompensationssignals wurde hier verzichtet.

Es zeigt sich, daß die zusätzliche Adaption des Volterra-Filters zweiter Ordnung (orange) im Frequenzbereich zwischen etwa $50 \mathrm{~Hz}$ und $200 \mathrm{~Hz}$ zu einer weiteren Reduktion der Fehlerleistung um bis zu 8 dB gegenüber der Kompensation mit dem linearen Modell (rot) führt. Dies entspricht einer zusätzlichen Reduktion der Störamplitude um etwa 60 \%. Die Koeffizienten des linearen FIR-Filters und die Koeffizientenmatrix des Volterra-Filters zweiter Ordnung $(N=60)$ sind in Abbildung 7.4 aufgetragen.

Dieses positive Ergebnis relativiert sich jedoch bei der Betrachtung der gemessenen Störprofile (Abb. 7.5). Anstelle der anhand der Wandschubspannungsmessungen erwarteten zusätzlichen Störreduktion durch die nichtlineare Nachbildung ergibt sich sogar eine geringfügige Vergrößerung der Störung. Diese Vergrößerung des RMS-Wertes der Geschwindigkeitsschwankung im wandnahen Maximum kann mit dem Oberflächenhitzdraht offenbar nicht detektiert und damit vom Algorithmus nicht berücksichtigt werden. 


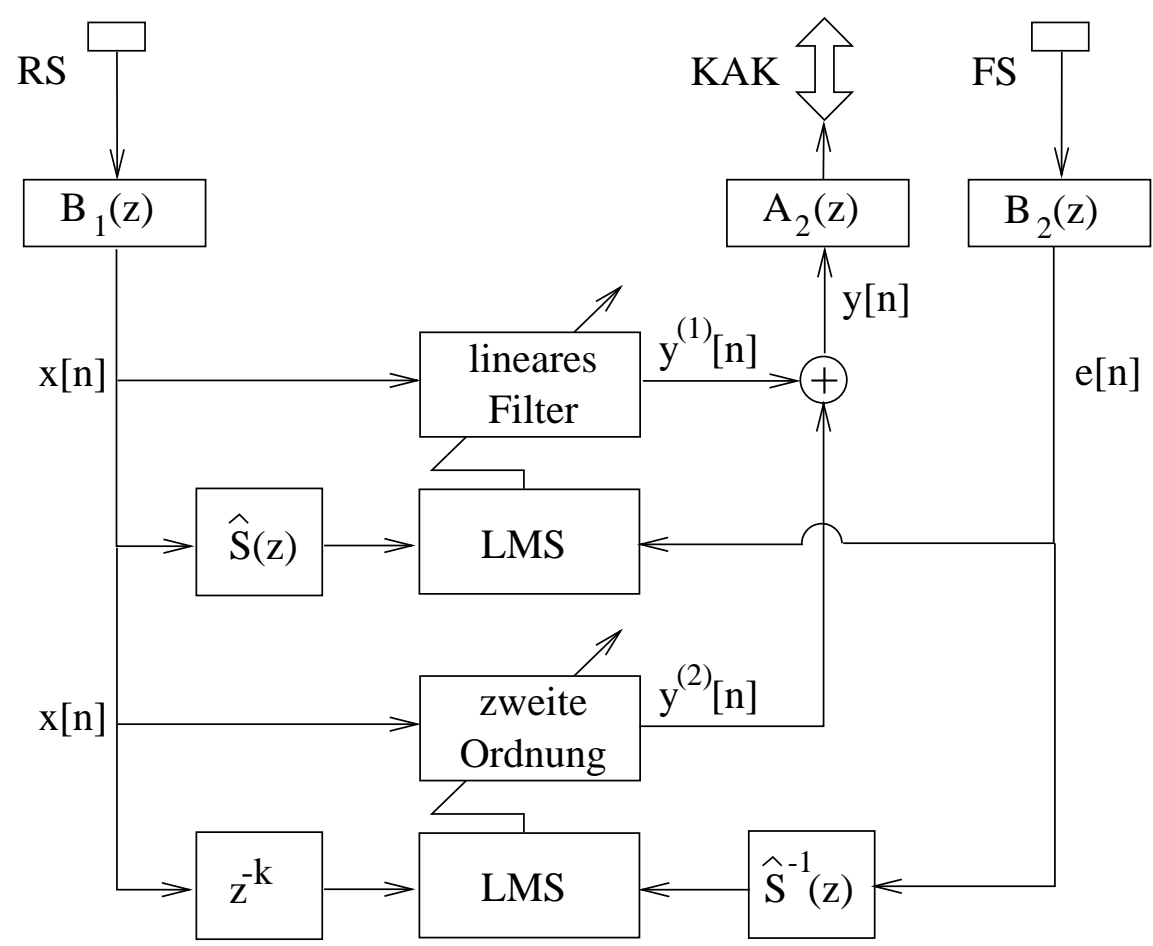

Abbildung 7.2: Signalverarbeitung zur dynamischen Stabilisierung mit einem nichtlinearen Modell 


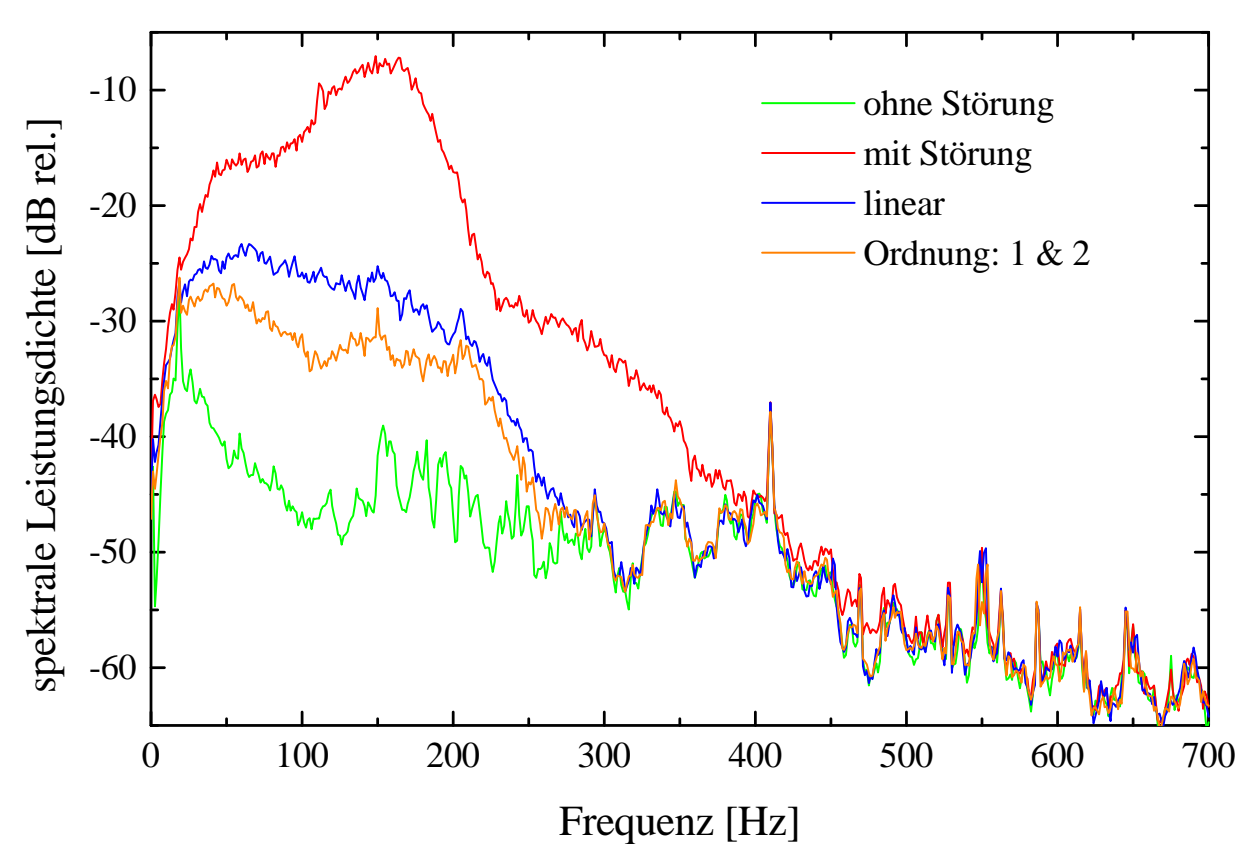

Abbildung 7.3: Spektrale Leistungsdichte des Fehlersensorsignals (Oberflächenhitzdraht), Amplitude: 0,8

Daher wurde ein weiteres Experiment durchgeführt, wobei als Fehlersensor eine im wandnahen Störmaximum positionierte Hitzdrahtsonde verwendet wurde. Vergleicht man die in Abbildung 7.6 dargestellte spektrale Leistungsdichte des mit der Hitzdrahtsonde aufgenommenen Fehlersignals (mit Störung, rot) mit den Ergebnissen aus Abbildung 7.3, so zeigen sich sowohl im Bereich der tieffrequenten Störanteile um $75 \mathrm{~Hz}$ als auch für die höherharmonischen Störungen $(300 \mathrm{~Hz})$ deutliche Unterschiede. Während die höherharmonischen Anteile bei der Hitzdrahtmessung nicht so deutlich aufgelöst werden, ist die Leistung der tieffrequenten Anteile bei dieser Messung deutlich höher.

Läßt man das lineare Filter adaptieren, so ergibt sich eine deutliche Reduktion der spektralen Fehlerleistung (blau) und auch in diesem Experiment führt die zusätzliche Adaption des Filters zweiter Ordnung zu einer weiteren Verringerung des Fehlers (orange). Die in Abbildung 7.7 dargestellten Koeffizienten der Filter erster und zweiter Ordnung zeigen, daß die Aufnahme des Fehlersignals mit dem Hitzdraht insbesondere für das nichtlineare Filter zu einer deutlichen Änderung der nachgebildeten Korrelationen geführt hat (vgl. Abb. 7.4).

Die bei diesem Experiment gemessenen Störprofile sind in Abbildung 7.8 dargestellt. Für die Kompensation mit einem linearen Modell (blau), ist das Störprofil weitgehend identisch mit dem Ergebnis aus Abb. 7.5. Betrachtet man jedoch das Störprofil nach Adaption der Filter erster und zweiter Ordnung, so zeigt sich hier 


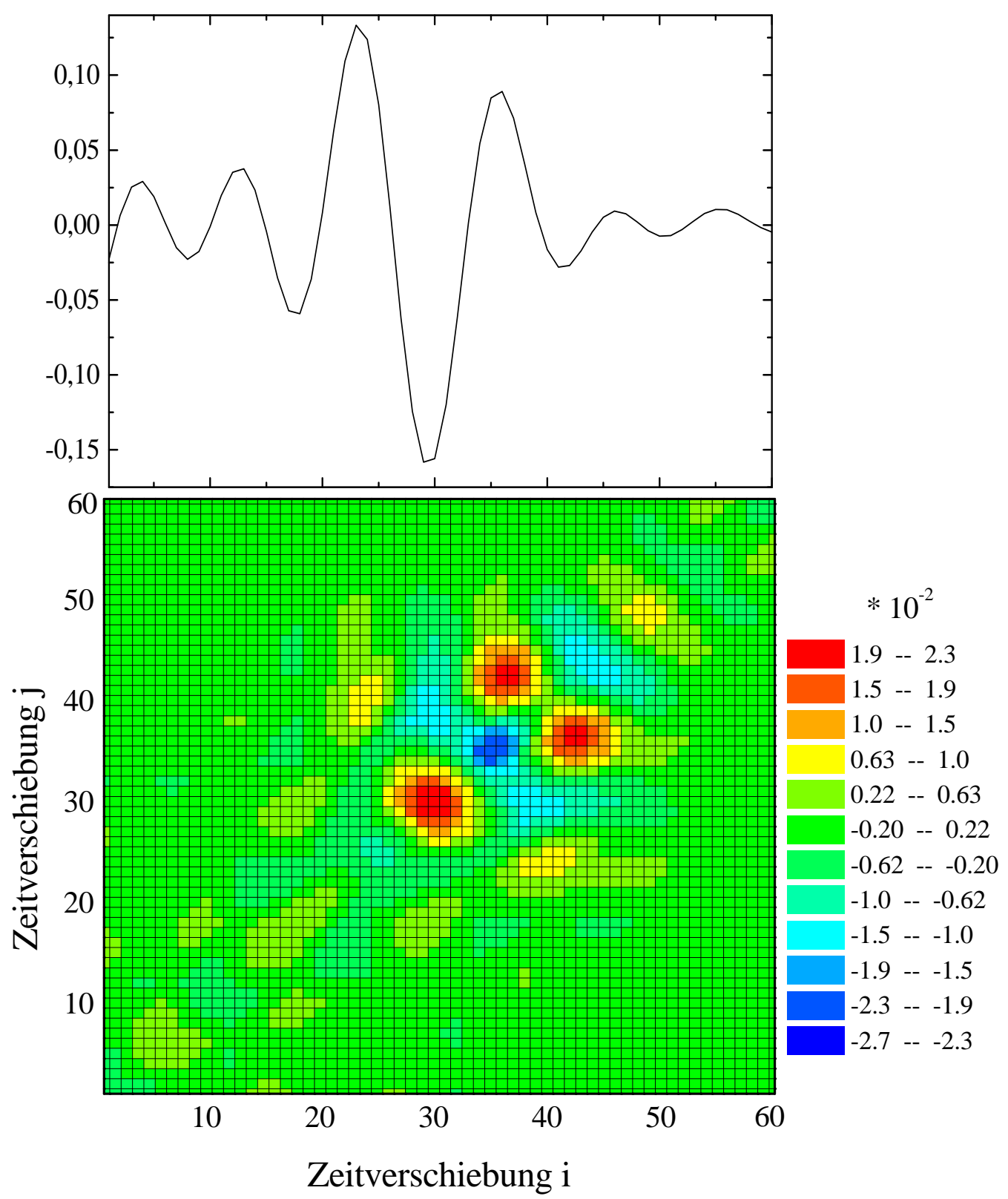

Abbildung 7.4: Koeffizientenvektor des linearen FIR-Filters (oben) und Koeffizientenmatrix des Volterra-Filters zweiter Ordnung (unten), Oberflächenhitzdraht als Fehlersensor 


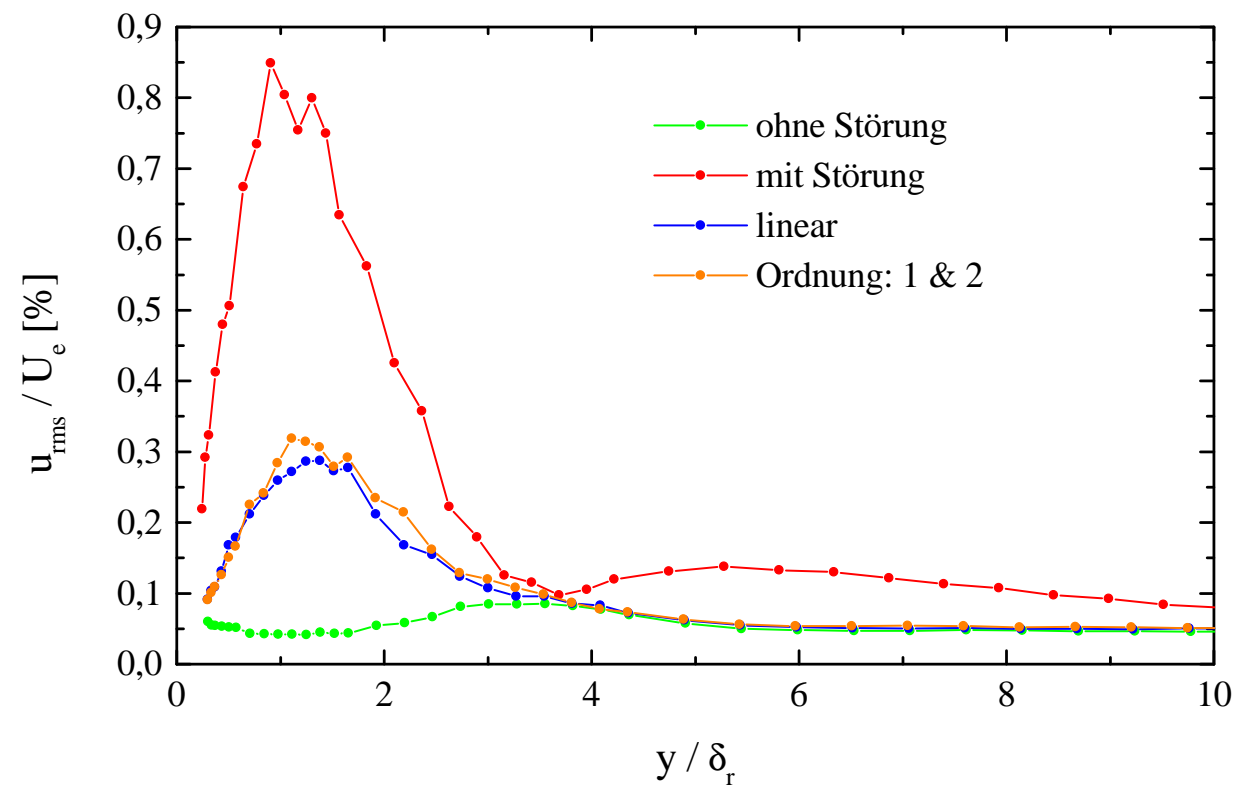

Abbildung 7.5: Störprofile, Amplitude: 0,8, Oberflächenhitzdraht als Fehlersensor

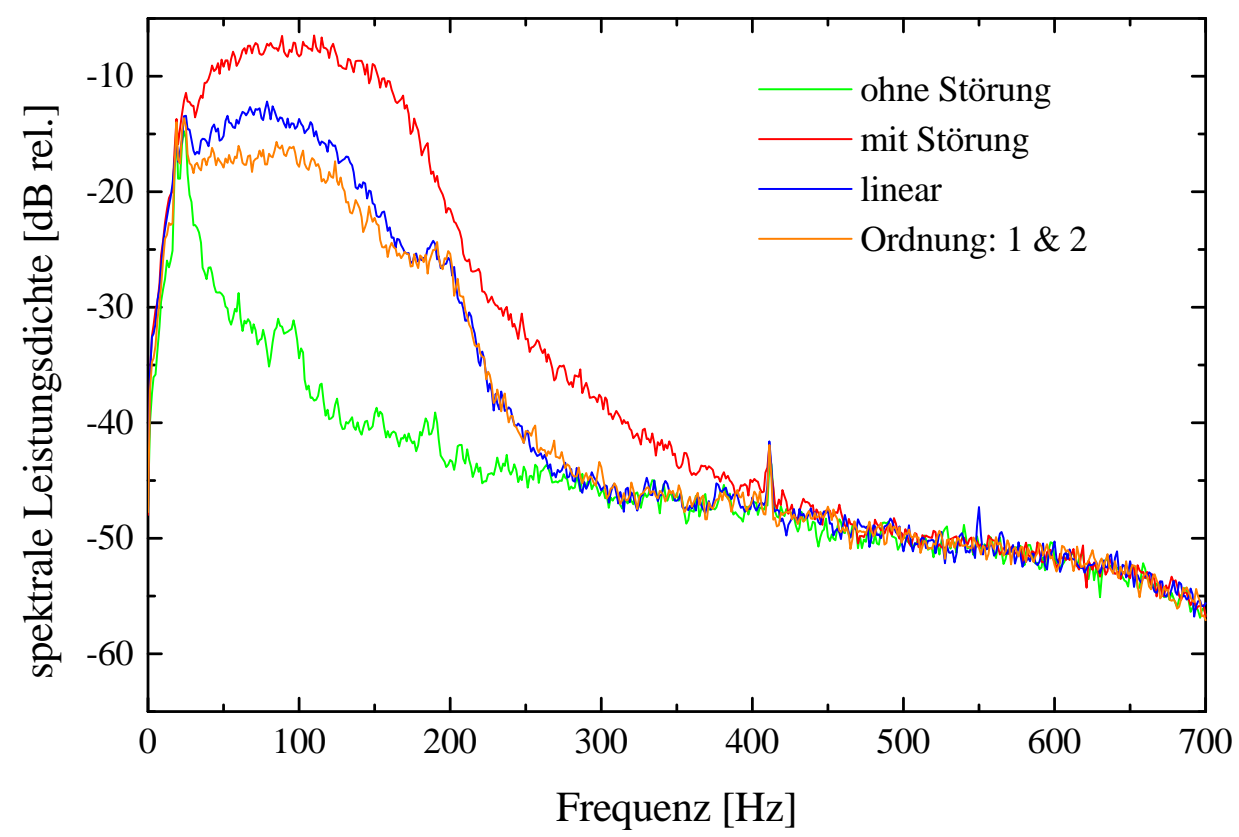

Abbildung 7.6: Spektrale Leistungsdichte des Fehlersensorsignals (Hitzdraht im Maximum der TS-Störwelle), Amplitude: 0,8, Oberflächenhitzdraht als Fehlersensor 


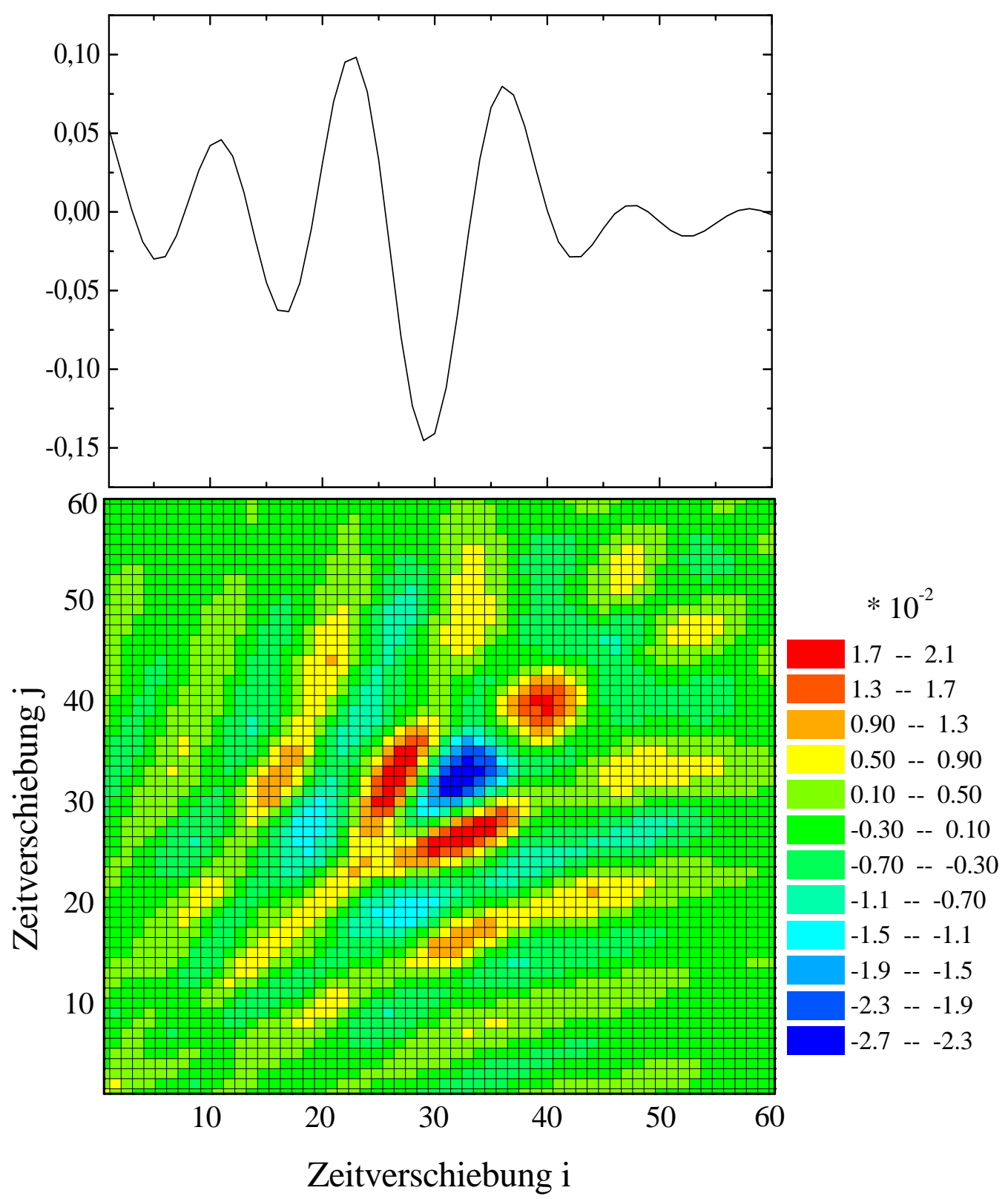

Abbildung 7.7: Koeffizientenvektor des linearen FIR-Filters (oben) und Koeffizientenmatrix des Volterra-Filters zweiter Ordnung (unten), Hitzdraht als Fehlersensor 


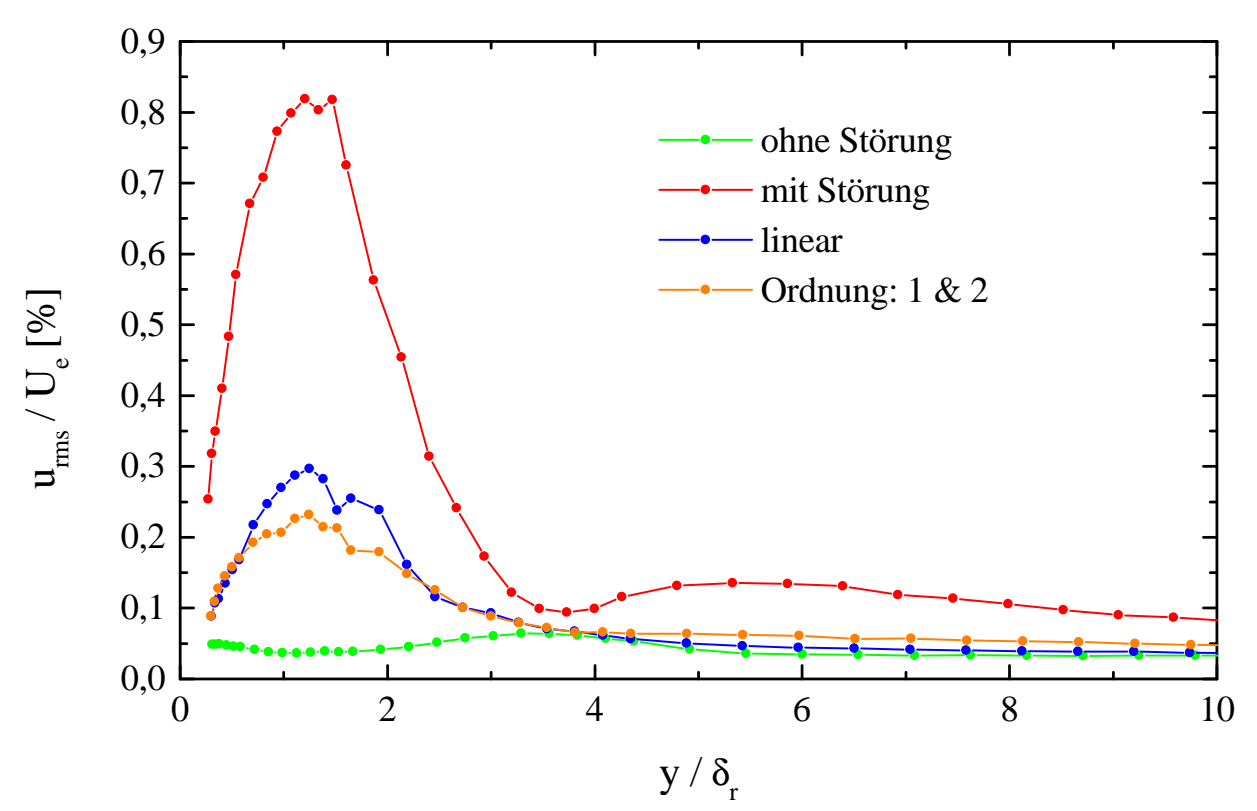

Abbildung 7.8: Störprofile, Amplitude: 0,8, Hitzdraht als Fehlersensor

auch in der Profilmessung eine zusätzliche Reduktion der Störung durch die nichtlineare Nachbildung. Allerdings weist der Bereich des Nebenmaximums $(\eta \approx 6)$ eine geringfügige Vergrößerung des RMS-Wertes auf.

Beachtet man jedoch, daß die linear nichtkompensierbare Reststörung (blau) nicht mehr die Profilform einer TS-Welle hat (kein Nebenmaximum), so läßt sich dieses Ergebnis am einfachsten dadurch erklären, daß im Fall des nichtlinearen Modells eine im Vergleich zur linearen Modellierung etwas größere Kompensations-TS-Welle generiert wird, die im Bereich des wandnahen Maximums zu einer verbesserten Störreduktion führt, im Bereich des Nebenmaximums jedoch als zusätzliche Störung auftritt. 


\section{Kapitel 8}

\section{Zusammenfassung \& Ausblick}

Es wurde die dynamische Stabilisierung einer laminaren Plattengrenzschicht untersucht, wobei Grenzschichtstörungen (z.B. TS-Wellen), deren Anfachung stromab den laminar-turbulenten Umschlag einleitet, durch künstlich angeregte, gegenphasige Störungen gezielt kompensiert werden. Die zu kompensierenden Primärstörungen werden mit einem Referenzsensor aufgenommen, um hieraus im Computer ein geeignetes Anregungssignal für den weiter stromab positionierten Kompensationsaktuator zu berechnen. Hierfür muß im wesentlichen die Übertragungsfunktion der physikalischen Ausbreitungsprozesse zwischen der Position des Referenzsensors und der Position des Kompensationsaktuators nachgebildet werden. Für diese Nachbildung wurden adaptive Digitalfilter eingesetzt, deren Koeffizienten über einen Algorithmus (LMS, least mean square) so eingestellt werden, daß die Leistung des stromab, außerhalb des Aktuatornahfeldes, aufgenommenes Fehlersignals minimal wird.

Die zweidimensionalen Primärstörungen wurden breitbandig mit einem Schlitzaktuator (Störaktuator) künstlich angeregt. Somit konnte die Störamplitude variiert und der Kompensationserfolg in Abhängigkeit von der Störamplitude untersucht werden. Hiefür wurde sowohl die spektrale Leistungsdichte des Fehlersignals (Funktion der Wandschubspannung), als auch der RMS-Wert der Schwankungsgeschwindigkeit in Abhängigkeit vom Wandabstand (Störprofil) aufgenommen.

Bei den ersten Experimenten wurde zur Nachbildung der Übertragungsfunktion ein FIR-Filter eingesetzt. Es zeigen sich sehr gute Kompensationsergebnisse, wobei allerdings für größere Störamplituden zunehmend nichtkompensierbare Reststörungen auftreten. Ihre Lage im Frequenzbereich weist darauf hin, daß diese Störanteile über nichtlineare Ausbreitungsprozesse generiert werden (Summenund Differenzfrequenzen), deren Übertragungsverhalten mit einem FIR-Filter, das ein lineares Modell darstellt, nicht nachgebildet werden kann. Verfolgt man die Störentwicklung stromab, so zeigt sich für hohe Störamplituden, daß der Störanteil, der linear mit dem Anregungssignal korreliert ist, mit zunehmender 


\section{Lauflänge abnimmt.}

Es wurde daher anhand eines adaptiven Volterra-Filters untersucht, inwieweit die mit dem Fehlersensor aufgenommenen Signale über höhere Korrelationen mit dem Signal vom Referenzsensor verknüpft sind. Dabei zeigt sich, daß schon die zusätzliche Adaption eines Volterra-Filters zweiter Ordnung, gegenüber der Modellierung mit einem rein linearen Modell, zu einer deutlich verbesserten Nachbildung der Übertragungsprozesse führt.

Da der Volterra-Ansatz insbesondere für höhere Ordnungen der Nichtlinearität sehr rechenintensiv ist, wurde versucht, daß nichtlineare Modell durch die Integration von Vorwissen über den nachzubildenen Übertragungsprozess so zu modifizieren, daß eine ähnlich gute Nachbildung mit weniger anzupassenen Koeffizienten erreicht wird.

Die viskose Instabilität führt zu einer bandpaßartigen Übertragungscharakteristik, die beim sogenannten Kautz-Filter über die geeignete Positionierung eines komplex konjugierten Polpaars in der komplexen Ebene als Vorwissen in die Filterstruktur integriert wird. Es konnte gezeigt werden, daß bei identischen linearen Modellierungsqualitäten für ein Kautz-Filter nur etwa halb so viele zu adaptierende Koeffizienten benötigt werden, wie bei Verwendung eines FIR-Filters.

Ausgehend von diesem Ergebnis wurde das Kautz-Filter zur Nachbildung höherer Korrelationen erweitert. Die mit dieser Filterstruktur unter Berücksichtigung von Nichtlinearitäten bis zur vierten Ordnung durchgeführten Streckenerkennungsexperimente zeigen gegenüber der Nachbildung mit einem Volterra-Filter erster und zweiter Ordnung eine weitere Verbesserung der Nachbildung, wobei im Vergleich mit dem Volterra-Filter deutlich weniger Koeffizienten benötigt werden.

Im nächsten Schritt wurde untersucht, inwieweit sich die Ergebnisse aus der Streckenerkennung auf das Schema der dynamischen Stabilisierung übertragen lassen, das heißt, ob sich durch die Integration eines nichtlinearen Modells für große Störamplituden eine verbesserte Kompensation erreichen läßt.

Es zeigt sich, daß die nichtlineare Erweiterung zu einer zusätzlichen Reduktion der Leistung des mit einem Oberfächenhitzdraht aufgenommenen Fehlersignal (Funktion der Wandschubspannung) führt. Das mit einer Hitzdrahtsonde aufgenommene Störprofil jedoch läßt im Gegensatz dazu eine geringfügige Erhöhung des RMS-Wertes im Bereich des wandnahen Maximums $(\eta \approx 1)$ erkennen. Will man die Reststörungen, deren Profilform sich hier von der Profilform einer TSWelle unterscheidet, kompensieren, ist daher die Wandschubspannung anscheinend kein geeignetes Fehlerkriterium.

Deshalb wurde in einem weiteren Experiment eine im Maximum der TS-Welle positionierte Hitzdrahtsonde als Fehlersensor verwendet. Das anhand der Minimierung dieses Fehlersignals angepaßte, nichtlineare Filter führt im Vergleich mit einer linearen Modellierung sowohl zu einer weiteren Reduktion der spektralen 
Fehlerleistung, als auch zu einer Verminderung des RMS-Wertes der Schwankungsgeschwindigkeit im wandnahen Bereich. In diesem Fall läßt sich jedoch im Bereich des Nebenmaximums des TS-Profils eine leichte Störanhebung als Folge der nichtlinearen Modellierung erkennen. Dies wird darauf zurückgeführt, daß im Fall des nichtlinearen Modells eine im Vergleich zur linearen Modellierung etwas größere Kompensations-TS-Welle generiert wird, die im Bereich des Nebenmaximums als zusätzliche Störung auftritt, da das Profil der nichtkompensierbaren Störungen dieses Nebenmaximum nicht aufweist.

In weiterführenden Experimenten sollte insbesondere das Verhalten der nichtkompensierbaren Reststörungen bei der weiteren Stromabausbreitung und ihr Einfluß auf den laminar-turbulenten Umschlag untersucht werden. Um die Strömung über eine größere Lauflänge laminar zu erhalten, könnten mehrere der hier untersuchten Einzelsysteme zur dynamischen Stabilisierung (Sensor-Aktuator-Sensor) stromab gestaffelt werden. Hierbei stellt sich die Frage, ob es andere Sensoren gibt, die eine Detektion der beobachteten Reststörungen ermöglichen ohne ihrerseits die Strömung zu beeinflussen. 


\section{Anhang A}

\section{Der filtered-X LMS-Algorithmus}

In diesem Abschnitt wird anhand des Aufbaus der dynamischen Stabilisierung der sogenannte filtered-X LMS-Algorithmus hergeleitet.

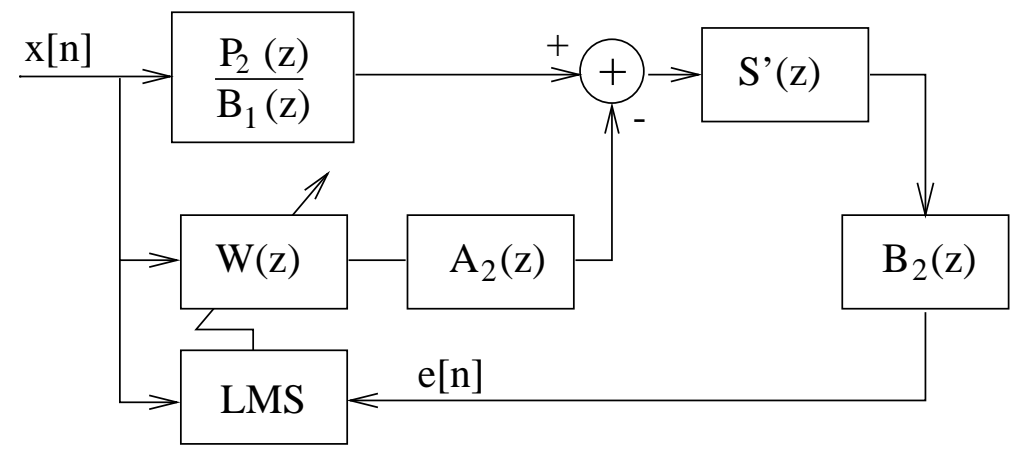

Abbildung A.1: Schematische Darstellung der Signalverarbeitung

In Abbildung A.1 ist noch einmal die Signalverarbeitung aus Abbildung 3.4 dargestellt. Das Summationszeichen bezeichnet die Überlagerung der Störung und des berechneten gegenphasigen Signals am Ort des Aktuators. Verschiebt man diese Summation durch die Strecken $S^{\prime}(z)$ und $B_{2}(z)$ und faßt die Strecken $A_{2}(z)$, $S^{\prime}(z)$ und $B_{2}(z)$ zur Sekundärstrecke $S(z)$ zusammen, so erhält man die in Abbildung A.2 gezeigte äquivalente Darstellung. Diese Umformungen sind natürlich nur aufgrund der vorausgesetzten Linearität und bei langsamer zeitlicher Änderung der Übertragungsfunktionen möglich, also unter der Annahme, daß es sich im wesentlichen um LTI-Systeme (linear, time-invariant) handelt.

Zieht man jetzt noch die Sekundärstrecke vor das adaptive Filter und die zu erkennende Strecke, so erhält man den Aufbau aus Abbildung A.3, allerdings ohne die gestrichelt eingetragene Sekundärstrecke.

Beinhaltet die Sekundärstrecke eine Verzögerung, so bewirkt diese eine Verschie- 
bung der Eingangssignale für das Filter und den LMS-Algorithmus gegeneinander, wodurch sich an für den Adaptionsprozeß „falschen“ Stellen Korrelationen ergeben.

Um dieses Problem zu lösen, kann man zusätzlich die gestrichelt eingetragene Filterung des Eingangssignals mit einem digitalen Filter durchführen, dessen Übertragungsverhalten dem der Sekundärstrecke entspricht. Man erhält dadurch den sogenannten filtered-X LMS-Algorithmus. In dieser Form entspricht die dynamische Stabilisierung einer Streckenerkennung zwischen dem mit der Sekundärstrecke gefilterten Referenzsignal $x^{\prime}[n]$ und dem Signal des Fehlersensors $d[n]$.

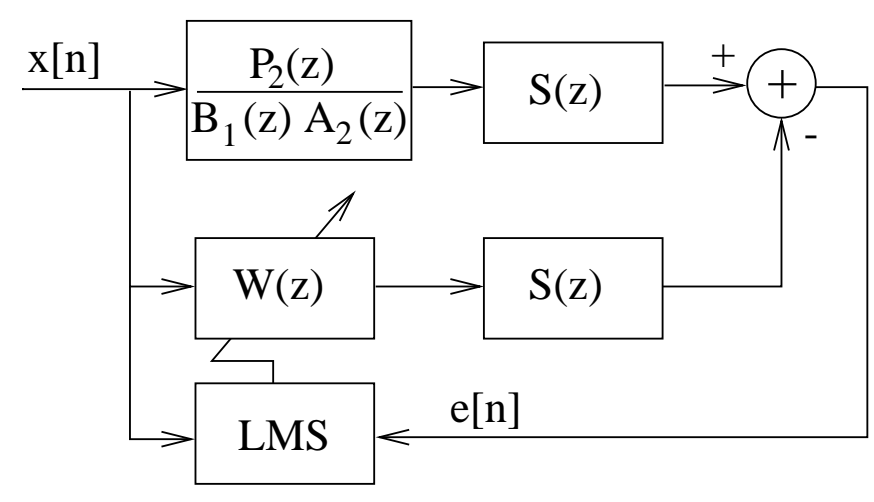

Abbildung A.2: ̈̈quivalente Darstellung

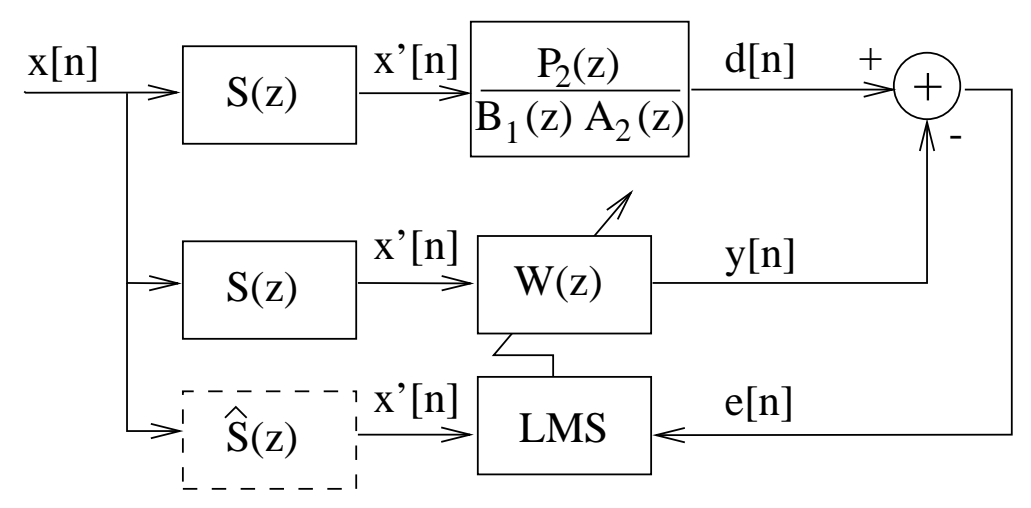

Abbildung A.3: filtered-X LMS 


\section{Literaturverzeichnis}

[1] Alexander, S. Thomas: Adaptive Signal Processing. Springer, New York, 1986.

[2] Baumann, Martin: Aktive Dämpfung von Tollmien-Schlichting Wellen in einer Flügelgrenzschicht. Fortschr.-Ber. VDI Reihe 7 Nr. 372, VDI Verlag, Düsseldorf, 1999.

[3] Belt, H. J. W. und A.C. Den Brinker: Laguerre Filters with Adaptive Pole Optimization. In: Proc. IEEE Int. Symp. on Circuits and Systems, Band II, Seiten 977-980, Atlanta, GA, May 1996.

[4] Bushnell, Dennis M.: Introduction. In: Bushnell, D.M. und J.N. HeFNER (Herausgeber): Viscous Drag Reduction in Boundary Layers, Progress in Astronautics and Aeronautics, Band 123. AIAA, 1990.

[5] Den Brinker, Albertus C.: Optimality Conditions for a Specific Class of Truncated Kautz Series. IEEE Transactions on Automatic Control-II, 43(8):597-600, 1996.

[6] den Brinker, Albertus C., Frank P.A. Benders und Tomás A.M. OliveIRA: Optimality Conditions for Truncated Kautz Series. IEEE Transactions on Automatic Control-II, 43(2):117-122, 1996.

[7] Eckelmann, H.: Einführung in die Strömungsmeßtechnik. Teubner, Stuttgart, 1997.

[8] Emanuel, Harald und Caspar Steineke: Turbo-Talker; Interface zwischen PC-BUS und Motorolas DSP56002EVM. ELRAD, 6:28-30, 1996.

[9] Evert, F., D. Ronneberger und F.-R. Grosche: Dynamische Stabilisierung einer laminaren Plattengrenzschicht. ZAMM, 79, Suppl.2:603-604, 1999.

[10] Evert, F., D. Ronneberger und F.-R. Grosche: Application of linear and nonlinear adaptive filters for the compensation of disturbances in the laminar boundary layer. ZAMM, 80, Suppl.1:85-88, 2000. 
[11] Fejzo, Zoran und Hanoch Lev-Ari: Adaptive Nonlinear WienerLaguerre-Lattice Models. In: Proceedings of ICASSP, Seiten 977-980, Detroit, MI, May 1995.

[12] Fejzo, Zoran und Hanoch Lev-Ari: Adaptive Laguerre-Lattice Filters. IEEE Transactions on Signal Processing, 45(12):3006-3016, 1997.

[13] Gölling, Burkhard: Dynamisch-aktive Dämpfung einer künstlich angeregten zweidimensionalen Welle im Bereich linearer und nichtlinearer Instabilitätsentwicklung in einer Blasius-Grenzschichtströmung. Diplomarbeit, Institut f. Angewandte Mechanik und Strömungsphysik, Universität Göttingen, 1996.

[14] Griffith, David W. und Gonzalo R. Arce: Partially Decoupled Volterra Filters : Formulation and LMS Adaption. IEEE Transactions on Signal Processing, 45(6):1485-1494, 1997.

[15] Haykin, Simon: Adaptive Filter Theory. Prentice Hall, Englewood Cliffs, NJ, dritte Auflage, 1996.

[16] Jahnke, ERIC. persönliche Mitteilung, 2000. DLR Göttingen.

[17] Joslin, Ronald D.: Aircraft Laminar Flow Control. Annu. Rev. Fluid. Mech., 30:1-29, 1998.

[18] Kessler, Manuel: Pentium optimieren. Toolbox, 2, 1997.

[19] Kuo, Sen M. und Dennis R. Morgan: Active Noise Control Systems. JOHN WILEY \& SONS, INC., Canada, 1996.

[20] Lacroix, ArIld: Digitale Filter. R. Oldenbourg, München, vierte Auflage, 1996.

[21] LADD, D.M.: Control of Natural Laminar Instability Waves on an Axisymmetric Body. AIAA JOURNAL, 28(2):367-369, 1990.

[22] Ladd, D.M. und E.W. Hendricks: Active Control of 2-D Instability Waves on an Axisymmetric Body. Experiments in Fluids, 6:69-70, 1988.

[23] Liepmann, H.W. und G.L. Nosenchuk: Active Control of Laminarturbulent Transition. J. Fluid Mech., 118:201-204, 1982.

[24] Liepmann, H.W. und G.L. Nosenchuk: Control of Laminar-instability Waves Using a New Technique. J. Fluid Mech., 118:187-200, 1982.

[25] Lindskog, Peter: Methods, Algorithms and Tools for System Identification Based on Prior Knowledge. Doktorarbeit, Linköping University, Department of Electrical Engineering, 1996. 
[26] Mathews, V. John: Adaptive Polynomial Filters. IEEE Signal Processing Magazine, 8(3):10-26, 1991.

[27] Milling, Robert W.: Tollmien-Schlichting Wave Cancellation. Phys. Fluids, 24(5):979-981, 1981.

[28] Ninness, Brett und Fredrik Gustafsson: A Unifying Construction of Orthonormal Bases for System Identification. IEEE Transactions on Automatic Control, 42(3), 1997.

[29] Oppenheim, A. V. und R. W. Schafer: Zeitdiskrete Signalverarbeitung. R. Oldenbourg, München, dritte Auflage, 1999.

[30] Pupator, P. und W. Saric: Control of Random Disturbances in a Boundary Layer, 1989. AIAA Paper 89-1007.

[31] Raguse, AndReas: Experimentelle Untersuchungen zur aktiven dynamischen Beeinflussung des laminar-turbulenten Umschlags in einer Plattengrenzschicht. Doktorarbeit, Math.-Nat. Fak. Univ. Göttingen, 1998.

[32] Schetzen, M.: The Volterra and Wiener Theories of Nonlinear Systems. JOHN WILEY \& SONS, INC., New York, 1980.

[33] Schlichting, H. und K. Gersten: Grenzschicht-Theorie. SpringerVerlag, Berlin Heidelberg, 1997.

[34] Slock, Dirk T. M. und Thomas Kailath: Numerically Stable Fast Transversal Filters for Recursive Least Squares Adaptive Filtering. IEEE Transactions on Signal Processing, 39(1):92-114, 1991.

[35] S. S.Narayan, A.M. Peterson und M.J. Narasimha: Transform Domain LMS Algorithm. IEEE Trans. Acoust., Speech, Signal Processing, 31(3):609-615, 1983.

[36] Strauch, Paul und Bernard Mulgrew: Active Control of Nonlinear Noise Processes in a Linear Duct. IEEE Transactions on Signal Processing, 46(9):2404-2412, 1998.

[37] Thomas, Andrew S.W.: The Control of Boundary-layer Transition Using a Wave-superposition Principle. J. Fluid Mech., 137:233-250, 1983.

[38] Thomas, Andrew S.W.: Active Wave Control of Boundary-Layer Transition. In: Bushnell, D.M. und J.N. Hefner (Herausgeber): Viscous Drag Reduction in Boundary Layers, Progress in Astronautics and Aeronautics, Band 123. AIAA, 1990. 
[39] Widrow, Bernard: Adaptive Filters. In: Kalman, R. E. und N. De Claris (Herausgeber): Aspects of Network and System Theory, Seiten 16921716. Holt, Rinehart and Winston, New York, 1970.

[40] Widrow, Bernard und Samuel D. Stearns: Adaptive Signal Processing. Prentice Hall, Englewood Cliffs, NJ, 1985.

[41] Zhou, A. und F.-R. Grosche: Experimental Study on Active Control of Boundary Layer by a Multiple Slot Blowing/Suction System, 1994. DLR-IB 223-94 A 33. 


\section{Danksagung}

Zunächst möchte ich Herrn Prof. Dr. D. Ronneberger und Herrn Dr. F.-R. Grosche (DLR Göttingen) für die Betreuung dieser Arbeit und ihre zahlreichen Anregungen danken.

Die Diskussionen mit Herrn Prof. Dr. D. Ronneberger haben mir oft geholfen, ein gegebenes Problem unter einem neuen Blickwinkel zu betrachten und es in einen allgemeineren Zusammenhang zu stellen.

Einen herzlichen Dank allen (auch ehemaligen) Mitgliedern der Arbeitsgruppe Strömungsakustik, insbesondere Holger Opfer, Michael Hartmann, Andreas Pöthke und Hartwig Preckel, die mit nicht nur fachlichen Beiträgen zu einem sehr positiven Arbeitsklima beigetragen haben.

Bei den Mitgliedern der Institutsband und hier insbesondere bei Frau Gisa Kirschmann-Schröder möchte ich mich als Ihr Lieblingsschlagzeuger für viele lustige gemeinsame Proben und Auftritte bedanken.

Herrn Gero Timann danke ich für die Entwicklung des Programms zur Signalverarbeitung (PPDSP) und der Arbeitsgruppe um Herrn Dr. K. Lautscham für den Aufbau der Analog-Digital/Digital-Analog-Wandlereinheit.

Für die vielfältige Hilfe bei allen Fragen zu meinem Meßaufbau möchte ich mich herzlich bei Herrn D. Baumgarten (DLR Göttingen) bedanken.

Der Deutschen Forschungsgemeinschaft danke ich für die Förderung der vorliegenden Arbeit.

Mein besonderer Dank gilt meiner Frau Iris, die mir gerade in schwierigen Phasen sehr viel Rückhalt gegeben hat und meiner Tochter Lilli, die mir nach langen Meßreihen an der "Schnaufmaschine" immer wieder deutlich zu verstehen gegeben hat, daß es im Leben noch wichtigere Dinge gibt, als die 100\%-ige Kompensation von Grenzschichtstörungen.

Meinen Eltern danke ich für ihre Liebe und Freundschaft, mit der sie mich auf meinem bisherigen Lebensweg begleitet haben. 


\section{Lebenslauf}

25. Februar 1969

August 1975 - Juli 1978

August 1978 - Juli 1979

August 1979 - Mai 1989

30. Mai 1989

ab Oktober 1989

8. Februar 1993

ab Dezember 1994

20. Februar 1996

ab 1. August 1996 geboren in Göttingen als Sohn der Eheleute Heiko und Rosemarie Evert, geborene Bachler Grundschule Niendorfer Straße in Hamburg - Norderstedt

Grundschule Islandstraße in Hamburg - Meiendorf

Gymnasium Buckhorn in Hamburg - Volksdorf Ablegen der Abiturprüfung Studium der Physik an der GeorgAugust Universität zu Göttingen Ablegen der Diplomvorprüfung Diplomarbeit am Dritten Phys. Institut der Univ. Göttingen unter Anleitung von Dr. D. Guicking in Zusammenarbeit mit der Continental AG Hannover. Titel der Arbeit: Resonanzabsorber zur Minderung des Rollgeräusches von Kraftfahrzeugreifen Ablegen der Diplomprüfung Anstellung am Dritten Phys. Inst. als wissenschaftlicher Mitarbeiter und Beginn der vorliegenden Dissertation bei Prof. Dr. Ronneberger. Mitbetreuung des Praktikums zur Digitalen Signalverarbeitung 